\title{
A Theoretical Study on the Mechanism of the
}

\section{[2+1] Thermal Cycloaddition between Alkenes}

\section{and Stable Singlet (Phosphino)(silyl)carbenes}

\author{
Begoña Lecea ${ }^{a}$ Mirari Ayerbe, ${ }^{a}$ Ana Arrieta, ${ }^{b}$ Fernando P. Cossío, ${ }^{b}$ Vicenç Branchadell, ${ }^{c}$ \\ Rosa Ortuño ${ }^{c}$ and Antoine Baceiredo ${ }^{d}$
}

Farmazi Fakultatea, Euskal Herriko Unibertsitatea, P. K. 450, 01080 Vitoria-Gasteiz, Spain

Kimika Fakultatea , Euskal Herriko Unibertsitaea, P. K. 1071, 28080 San SebastiánDonostia, Spain

Departament de Química, Universitat Autònoma de Barcelona, 08193 Bellaterra, Barcelona, Spain

Laboratoire Hétérochimie Fondamentale et Appliquée, UMR 5069, Université Paul Sabatier, 118 Rue de Narbonne, 31062 Toulouse Cedex 04, France

\footnotetext{
${ }^{a}$ Farmazi Fakultatea, Euskal Herriko Unibertsitatea.

${ }^{\mathrm{b}}$ Kimika Fakultatea, Euskal Herriko Unibertsitatea.

${ }^{c}$ Universitat Autònoma de Barcelona

${ }^{\mathrm{d}}$ Université Paul Sabatier.
} 


\section{Supporting Information}

List of the Contents:

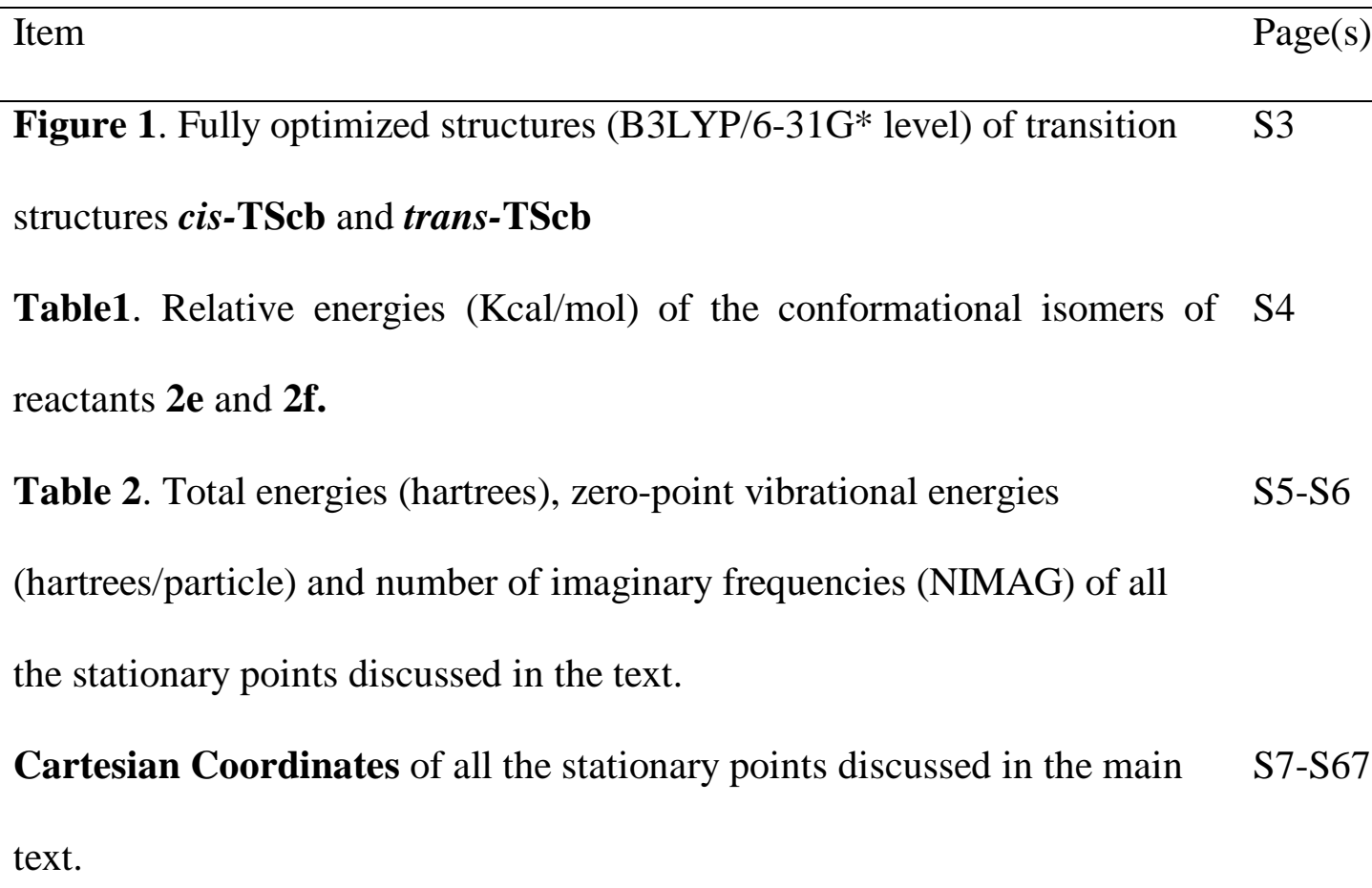




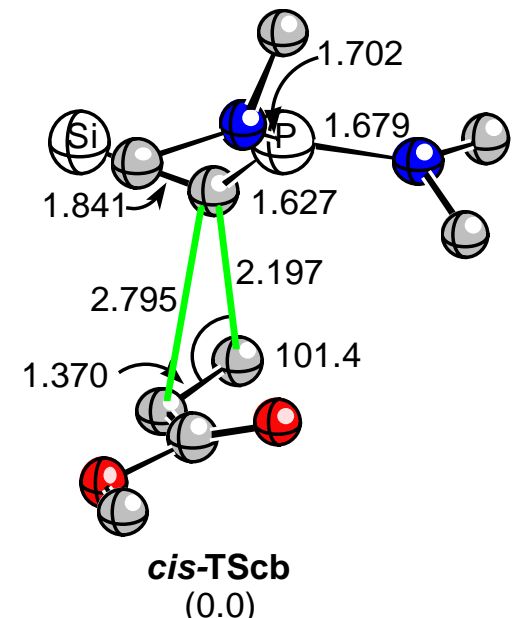

(0.0)

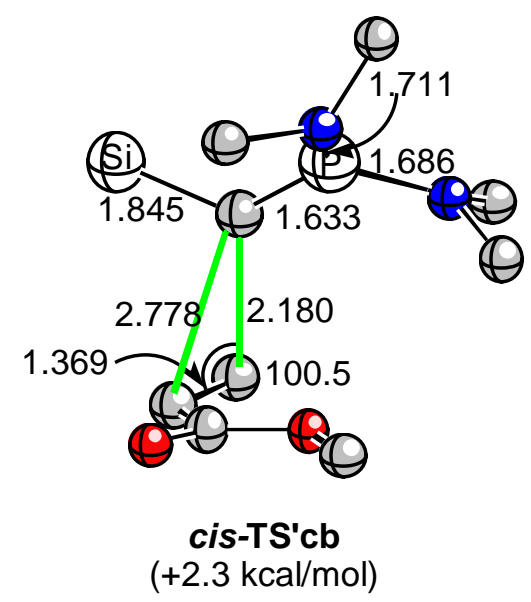

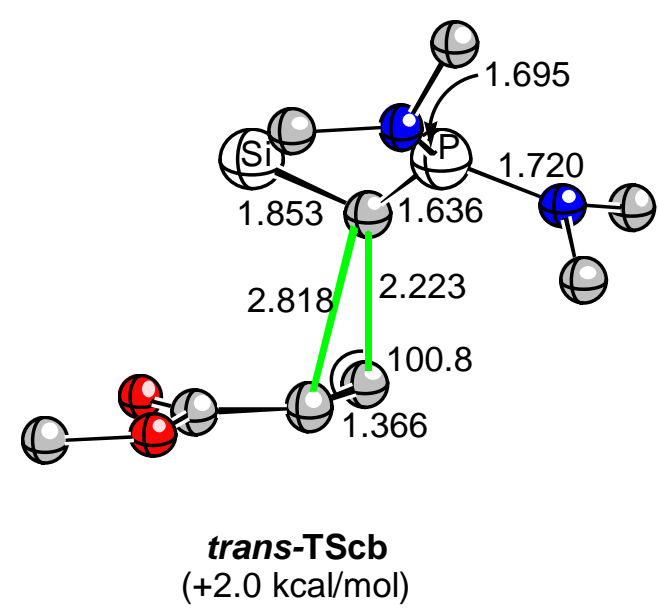

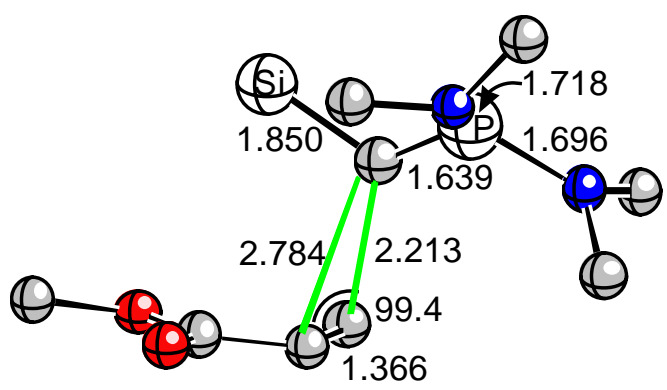

trans-TS'cb

$(+3.6 \mathrm{kcal} / \mathrm{mol})$

Figure 1. Fully optimized structures (B3LYP/6-31G* level) of transition structures cisTScb and trans-TScb (Scheme 2). Transition structures cis-TS'cb and trans-TS'cb correspond to the respective conformers possessing $s$-trans conformations about the methyl acrylate moiety. Numbers in parentheses are the relative energies. Hydrogen atoms have been omitted for clarity. Oxygen atoms are represented in red. See Figure 1 caption for additional details. 
Table 1. Relative energies (Kcal/mol) of the conformational isomers of reactants 2e and $2 f$.

\begin{tabular}{ccr}
\hline Compound & Conformation & Relative energy \\
\hline $\mathbf{2 e}$ & sc-sc & 0.00 \\
$\mathbf{2 e}$ & sc-st & 0.82 \\
$\mathbf{2 e}$ & st-st & 0.68 \\
$\mathbf{2 f}$ & sc-st & 6.41 \\
$\mathbf{2 f}$ & st-st & 7.51 \\
\hline
\end{tabular}


Table 2. Total energies (hartrees), zero-point vibrational energies (hartree/particle) and number of imaginary frequencies (NIMAG) of all the stationary points discussed in the text.

\begin{tabular}{lrrr}
\hline B3LYP/6-31G* & Energy & ZPVE & NIMAG \\
\hline 1a & -671.83534 & 0.04650 & 0 \\
\hline 1b & -782.57654 & 0.08425 & 0 \\
\hline 1c & -939.80454 & 0.19797 & 0 \\
\hline 1d & -1057.78546 & 0.28481 & 0 \\
\hline 1e & -1372.28928 & 0.51235 & 0 \\
\hline 2a & -78.58746 & 0.05124 & 0 \\
\hline 2b & -306.46776 & 0.09586 & 0 \\
\hline 2c & -325.90734 & 0,13623 & 0 \\
\hline 2d & -309.64826 & 0.13375 & 0 \\
\hline 2e & -534.34588 & 0.13938 & 0 \\
\hline 2f & -534.33541 & 0.13913 & 0 \\
\hline TSaa & -750.41002 & 0.09988 & 1 \\
\hline TSba & -861.25923 & 0.13649 & 1 \\
\hline TSca & -1018.37520 & 0.25049 & 1 \\
\hline cis-TScb & -1246.26442 & 0.19797 & 1 \\
\hline trans-TScb & -1246.25937 & 0.29457 & 1 \\
\hline cis-TS'cb & -1246.26089 & 0.29482 & 1 \\
\hline trans-TS'cb & -1246.25853 & 0.29441 & 1 \\
\hline cis-TScc & -1256.70492 & 0.33519 & 1 \\
\hline trans-TScc & -1265.69784 & 0.33504 & 1 \\
\hline cis-TScd & -1249.43904 & 0.33247 & 1 \\
\hline trans-TScd & -1249.43779 & 0.33247 & 1 \\
\hline cis-TSce-sc-sc & -1474.14228 & 0.33784 & 1 \\
\hline cis-TSce-sc-st & -1474.14288 & 0.33839 & 1 \\
\hline cis-TSce-st-sc & -1474.14288 & 0.33839 & 1 \\
\hline cis-TSce-st-st & -1474.13823 & 0.33809 & 1 \\
\hline trans-TSce-sc-sc & -1474.13815 & 0.33806 & 1 \\
\hline trans-TSce-sc-st & -1474.13725 & 0.33829 & 1 \\
\hline trans-TSce-st-sc & -1474.13240 & 0.33780 & 1 \\
\hline trans-TSce-st-sc & -1474.13166 & 0.33824 & 1 \\
\hline cis-TScf-sc-sc & -1474.13133 & 0.33862 & 1 \\
\hline cis-TScf-sc-st & -1474.12681 & 0.33877 & 1 \\
\hline cis-TScf-st-sc & -1474.12891 & 0.33827 & 1 \\
\hline cis-TScf-st-st & -1474.12113 & 0.33844 & 1 \\
\hline trans-TScf-sc-sc & -1474.12395 & 0.33766 & 1 \\
\hline trans-TScf-sc-st & -1474.12375 & 0.33799 & 1 \\
\hline trans-TScf-st-sc & -1474.12624 & 0.33799 & 1 \\
\hline trans-TScf-st-st & -1474.12435 & 0.33831 & 1 \\
\hline cis-TSdb & -1364.24333 & 0.38153 & 1 \\
\hline trans-TSdb & -1364.24043 & 0.38176 & 1 \\
\hline & & & \\
\hline
\end{tabular}




\begin{tabular}{lrll}
\hline cis-TS'db & -1364.23960 & 0.38163 & 1 \\
\hline trans-TS'db & -1364.23800 & 0.38164 & 1 \\
\hline cis-TSdd & -1367.41693 & 0.41949 & 1 \\
\hline trans-TSdd & -1367.41492 & 0.41917 & 1 \\
\hline cis-TSec & -1698.17961 & 0.65063 & 1 \\
\hline trans-TSec & -1698.25986 & 0.65498 & 1 \\
3aa & -750.53379 & 0.10517 & 0 \\
3ba & -861.25923 & 0.14118 & 0 \\
cis-3bb & -1089.13269 & 0.18483 & 0 \\
trans-3bb & -1089.13537 & 0.18508 & 0 \\
3ca & -1018.48246 & 0.25547 & 0 \\
cis-3cb & -1246.35672 & 0.29884 & 0 \\
trans-3cb & -1246.35478 & 0.29880 & 0 \\
cis-3cc & -1265.79850 & 0.33963 & 0 \\
trans-3cc & -1265.79598 & 0.33959 & 0 \\
cis-3cd & -1249.53170 & 0.33640 & 0 \\
trans-3cd & -1249.53325 & 0.33665 & 0 \\
cis-3ce & -1474.23152 & 0.34220 & 0 \\
trans-3ce & -1474.23172 & 0.34241 & 0 \\
cis-3cf & -1474.22260 & 0.34146 & 0 \\
trans-3cf & -1474.22430 & 0.34174 & 0 \\
cis-3db & -1364.32579 & 0.38515 & 0 \\
trans-3db & -1364.32897 & 0.38604 & 0 \\
cis-3dc & -1383.76613 & 0.42580 & 0 \\
trans-3dc & -1383.77030 & 0.42662 & 0 \\
cis-3dd & -1367.50408 & 0.42373 & 0 \\
trans-3dd & -1367.50269 & 0.42378 & 0 \\
cis-3ec & -1698.25968 & 0.65498 & 0 \\
trans-3ec & -1698.25773 & 0.65444 & 0 \\
$\mathbf{4}$ & -1383.75732 & 0.42683 & 0 \\
$\mathbf{5}$ & -1383.76613 & 0.42584 & 0 \\
\hline
\end{tabular}




\section{Cartesian Coordinates of all the Stationary Points Discussed in the Main Text.}

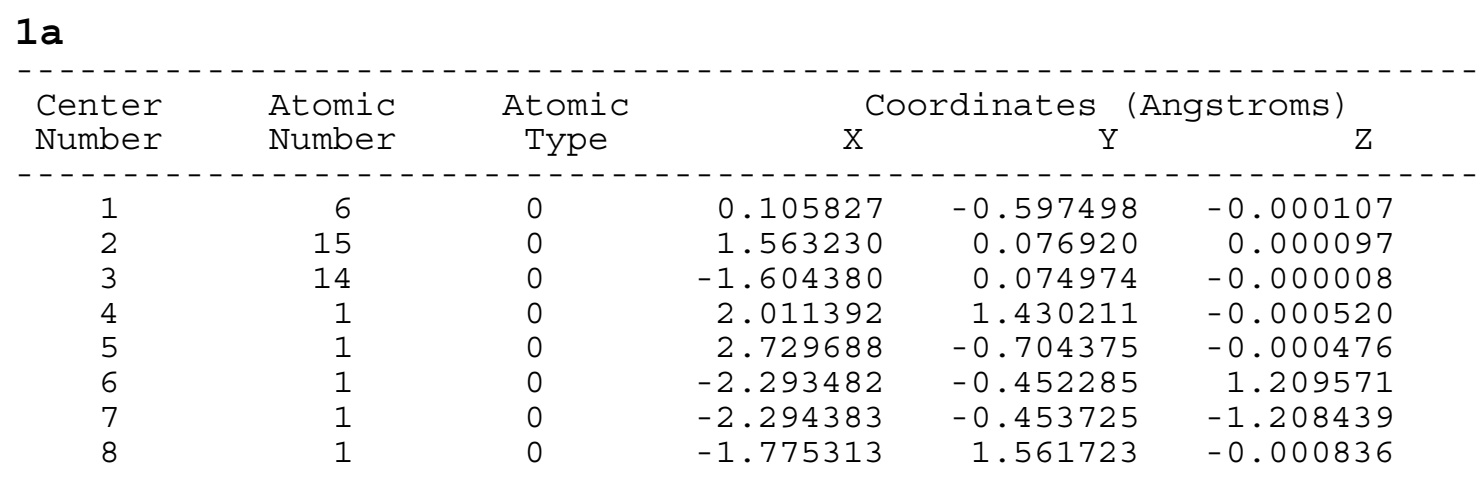

$1 \mathrm{~b}$

\begin{tabular}{|c|c|c|c|c|c|}
\hline \multirow{2}{*}{$\begin{array}{l}\text { Center } \\
\text { Number }\end{array}$} & \multirow{2}{*}{$\begin{array}{l}\text { Atomic } \\
\text { Number }\end{array}$} & \multirow{2}{*}{$\begin{array}{c}\text { Atomic } \\
\text { Type }\end{array}$} & \multicolumn{3}{|c|}{ Coordinates (Angstroms) } \\
\hline & & & $\mathrm{x}$ & $\mathrm{Y}$ & $\mathrm{Z}$ \\
\hline 1 & 6 & 0 & 0.693068 & -0.425469 & 0.039199 \\
\hline 2 & 15 & 0 & -0.816952 & -0.041799 & 0.013361 \\
\hline 3 & 7 & 0 & -1.724042 & 1.398757 & 0.056483 \\
\hline 4 & 14 & 0 & 2.442900 & 0.015970 & -0.010394 \\
\hline 5 & 7 & 0 & -2.113642 & -1.121668 & -0.082283 \\
\hline 6 & 1 & 0 & -1.148958 & 2.232849 & 0.112862 \\
\hline 7 & 1 & 0 & -2.437037 & 1.481835 & -0.664226 \\
\hline 8 & 1 & 0 & 3.096107 & -0.340289 & 1.283047 \\
\hline 9 & 1 & 0 & 3.137902 & -0.751481 & -1.084534 \\
\hline 10 & 1 & 0 & 2.761408 & 1.459567 & -0.255127 \\
\hline 11 & 1 & 0 & -2.842505 & -0.977244 & 0.611022 \\
\hline 12 & 1 & 0 & -1.807856 & -2.088646 & -0.112528 \\
\hline
\end{tabular}

$1 c$

\begin{tabular}{|c|c|c|c|c|c|}
\hline \multirow{2}{*}{$\begin{array}{l}\text { Center } \\
\text { Number }\end{array}$} & \multirow{2}{*}{$\begin{array}{l}\text { Atomic } \\
\text { Number }\end{array}$} & \multirow{2}{*}{$\begin{array}{l}\text { Atomic } \\
\text { Type }\end{array}$} & \multicolumn{3}{|c|}{ Coordinates (Angstroms) } \\
\hline & & & $\mathrm{X}$ & $\mathrm{Y}$ & $\mathrm{Z}$ \\
\hline & & & --------- & --------- & -------- \\
\hline 1 & 15 & 0 & -0.055921 & -0.117399 & -0.025930 \\
\hline 2 & 6 & 0 & 1.392864 & -0.719779 & -0.066871 \\
\hline 3 & 14 & 0 & 3.174489 & -0.438135 & 0.019607 \\
\hline 4 & 7 & 0 & -0.690319 & 1.462576 & -0.057875 \\
\hline 5 & 7 & 0 & -1.466816 & -1.042516 & 0.072973 \\
\hline 6 & 6 & 0 & 0.207716 & 2.516530 & -0.527841 \\
\hline 7 & 6 & 0 & -1.628165 & 1.905113 & 0.978099 \\
\hline 8 & 6 & 0 & -1.308340 & -2.432339 & 0.504480 \\
\hline 9 & 6 & 0 & -2.526076 & -0.858324 & -0.924144 \\
\hline 10 & 1 & 0 & 3.644145 & 0.983916 & 0.105484 \\
\hline 11 & 1 & 0 & 3.850102 & -1.015698 & -1.179988 \\
\hline 12 & 1 & 0 & 3.737430 & -1.131348 & 1.216204 \\
\hline 13 & 1 & 0 & 0.845846 & 2.136012 & -1.328367 \\
\hline 14 & 1 & 0 & 0.853951 & 2.905597 & 0.273340 \\
\hline 15 & 1 & 0 & -0.392865 & 3.345792 & -0.921245 \\
\hline 16 & 1 & 0 & -1.101689 & 2.314401 & 1.854567 \\
\hline 17 & 1 & 0 & -2.248162 & 1.067474 & 1.300309 \\
\hline 18 & 1 & 0 & -2.278889 & 2.689630 & 0.572221 \\
\hline 19 & 1 & 0 & -0.556965 & -2.495596 & 1.292819 \\
\hline 20 & 1 & 0 & -0.993987 & -3.089483 & -0.319142 \\
\hline 21 & 1 & 0 & -2.270071 & -2.788727 & 0.893151 \\
\hline 22 & 1 & 0 & -2.351914 & -1.474595 & -1.820122 \\
\hline
\end{tabular}




$\begin{array}{cccccc}23 & 1 & 0 & -2.579301 & 0.189225 & -1.223700 \\ 24 & 1 & 0 & -3.489706 & -1.149358 & -0.489106\end{array}$

\section{1d}

\begin{tabular}{|c|c|c|c|c|c|}
\hline \multirow{3}{*}{$\begin{array}{l}\text { Center } \\
\text { Number }\end{array}$} & \multirow{2}{*}{$\begin{array}{l}\text { Atomic } \\
\text { Number }\end{array}$} & \multirow{2}{*}{$\begin{array}{c}\text { Atomic } \\
\text { Type }\end{array}$} & \multicolumn{3}{|c|}{ Coordinates (Angstroms) } \\
\hline & & & $\mathrm{X}$ & $\mathrm{Y}$ & Z \\
\hline & & & --------- & -------- & -------- \\
\hline 1 & 6 & 0 & -0.615681 & -0.457166 & 0.064498 \\
\hline 2 & 15 & 0 & 0.904174 & -0.082586 & 0.028768 \\
\hline 3 & 14 & 0 & -2.401520 & -0.118266 & 0.000081 \\
\hline 4 & 7 & 0 & 1.770279 & 1.389593 & 0.066732 \\
\hline 5 & 7 & 0 & 2.180355 & -1.196176 & -0.073823 \\
\hline 6 & 6 & 0 & 2.721167 & 1.698591 & -1.005409 \\
\hline 7 & 6 & 0 & 1.036889 & 2.557654 & 0.549365 \\
\hline 8 & 6 & 0 & 3.247679 & -1.164219 & 0.930785 \\
\hline 9 & 6 & 0 & 1.820076 & -2.551129 & -0.491399 \\
\hline 10 & 6 & 0 & -3.142874 & -1.077542 & -1.460695 \\
\hline 11 & 6 & 0 & -3.199308 & -0.736261 & 1.607672 \\
\hline 12 & 6 & 0 & -2.865852 & 1.715134 & -0.211861 \\
\hline 13 & 1 & 0 & 3.476306 & 2.401911 & -0.632735 \\
\hline 14 & 1 & 0 & 3.222942 & 0.785916 & -1.330197 \\
\hline 15 & 1 & 0 & 2.223636 & 2.158087 & -1.874522 \\
\hline 16 & 1 & 0 & 1.756340 & 3.306741 & 0.902261 \\
\hline 17 & 1 & 0 & 0.412187 & 3.017377 & -0.231798 \\
\hline 18 & 1 & 0 & 0.388791 & 2.274417 & 1.381599 \\
\hline 19 & 1 & 0 & 2.975103 & -1.731062 & 1.835502 \\
\hline 20 & 1 & 0 & 4.156797 & -1.609928 & 0.508996 \\
\hline 21 & 1 & 0 & 3.462454 & -0.132959 & 1.214336 \\
\hline 22 & 1 & 0 & 1.394717 & -3.142892 & 0.332635 \\
\hline 23 & 1 & 0 & 1.081904 & -2.511278 & -1.293952 \\
\hline 24 & 1 & 0 & 2.722904 & -3.055301 & -0.857343 \\
\hline 25 & 1 & 0 & -4.228101 & -0.925374 & -1.521673 \\
\hline 26 & 1 & 0 & -2.708131 & -0.755210 & -2.414326 \\
\hline 27 & 1 & 0 & -2.957168 & -2.152578 & -1.357126 \\
\hline 28 & 1 & 0 & -4.283628 & -0.565972 & 1.600472 \\
\hline 29 & 1 & 0 & -3.025375 & -1.809759 & 1.742080 \\
\hline 30 & 1 & 0 & -2.788130 & -0.221381 & 2.484141 \\
\hline 31 & 1 & 0 & -3.954853 & 1.848784 & -0.257843 \\
\hline 32 & 1 & 0 & -2.495073 & 2.321549 & 0.623773 \\
\hline 33 & 1 & 0 & -2.441964 & 2.129146 & -1.135017 \\
\hline
\end{tabular}

\section{1e}

\begin{tabular}{|c|c|c|c|c|c|}
\hline \multirow{2}{*}{$\begin{array}{l}\text { Center } \\
\text { Number }\end{array}$} & \multirow{2}{*}{$\begin{array}{l}\text { Atomic } \\
\text { Number }\end{array}$} & \multirow{2}{*}{$\begin{array}{c}\text { Atomic } \\
\text { Type }\end{array}$} & \multicolumn{3}{|c|}{ Coordinates (Angstroms) } \\
\hline & & & $\mathrm{X}$ & $\mathrm{Y}$ & Z \\
\hline--- & & & --------- & -------- & -------- \\
\hline 1 & 6 & 0 & 1.492508 & -0.561684 & 0.130393 \\
\hline 2 & 14 & 0 & 3.270603 & -0.189944 & 0.093433 \\
\hline 3 & 6 & 0 & 3.947581 & -0.295494 & 1.864614 \\
\hline 4 & 15 & 0 & -0.035885 & -0.208809 & 0.055367 \\
\hline 5 & 7 & 0 & -1.303669 & -1.333349 & -0.002660 \\
\hline 6 & 6 & 0 & -2.405210 & -1.218930 & 0.992851 \\
\hline 7 & 6 & 0 & -3.787532 & -1.490663 & 0.377479 \\
\hline 8 & 7 & 0 & -0.850465 & 1.299314 & 0.014862 \\
\hline 9 & 6 & 0 & -0.191810 & 2.392646 & 0.780543 \\
\hline 10 & 6 & 0 & 0.280990 & 3.590533 & -0.054761 \\
\hline 11 & 6 & 0 & 4.156983 & -1.487752 & -0.972789 \\
\hline 12 & 6 & 0 & 3.734119 & 1.520201 & -0.606383 \\
\hline 13 & 6 & 0 & -1.865917 & 1.524892 & -1.048201 \\
\hline 14 & 6 & 0 & -1.272751 & 1.956039 & -2.404159 \\
\hline 15 & 6 & 0 & -1.103543 & -2.642895 & -0.686491 \\
\hline
\end{tabular}




\begin{tabular}{|c|c|c|c|c|c|}
\hline 16 & 6 & 0 & -0.077345 & -3.579502 & -0.025502 \\
\hline 17 & 6 & 0 & -3.017365 & 2.445690 & -0.620309 \\
\hline 18 & 6 & 0 & -1.026936 & 2.837972 & 1.994697 \\
\hline 19 & 6 & 0 & -0.790497 & -2.437271 & -2.174961 \\
\hline 20 & 6 & 0 & -2.181980 & -2.075328 & 2.250672 \\
\hline 21 & 1 & 0 & -2.302687 & 0.535729 & -1.209435 \\
\hline 22 & 1 & 0 & 0.716444 & 1.929892 & 1.180501 \\
\hline 23 & 1 & 0 & -2.081520 & -3.132596 & -0.630714 \\
\hline 24 & 1 & 0 & -2.394502 & -0.170201 & 1.302398 \\
\hline 25 & 1 & 0 & 5.239718 & -1.309019 & -0.998571 \\
\hline 26 & 1 & 0 & 3.990023 & -2.497601 & -0.580709 \\
\hline 27 & 1 & 0 & 3.794149 & -1.470190 & -2.007638 \\
\hline 28 & 1 & 0 & 4.823240 & 1.657077 & -0.638545 \\
\hline 29 & 1 & 0 & 3.356383 & 1.645498 & -1.628738 \\
\hline 30 & 1 & 0 & 3.317499 & 2.332566 & 0.001657 \\
\hline 31 & 1 & 0 & 5.028868 & -0.105902 & 1.880118 \\
\hline 32 & 1 & 0 & 3.471212 & 0.440941 & 2.522625 \\
\hline 33 & 1 & 0 & 3.770281 & -1.288556 & 2.293111 \\
\hline 34 & 1 & 0 & -3.473976 & 2.106578 & 0.314610 \\
\hline 35 & 1 & 0 & -3.790286 & 2.437759 & -1.397645 \\
\hline 36 & 1 & 0 & -2.699171 & 3.485041 & -0.491346 \\
\hline 37 & 1 & 0 & -0.909021 & 2.986401 & -2.396196 \\
\hline 38 & 1 & 0 & -2.042429 & 1.880906 & -3.182424 \\
\hline 39 & 1 & 0 & -0.440762 & 1.303337 & -2.689540 \\
\hline 40 & 1 & 0 & -0.431787 & 3.505815 & 2.629513 \\
\hline 41 & 1 & 0 & -1.320248 & 1.972845 & 2.598944 \\
\hline 42 & 1 & 0 & -1.934058 & 3.376538 & 1.706052 \\
\hline 43 & 1 & 0 & 0.855882 & 4.267936 & 0.587728 \\
\hline 44 & 1 & 0 & -0.549193 & 4.168939 & -0.472859 \\
\hline 45 & 1 & 0 & 0.932232 & 3.269070 & -0.872064 \\
\hline 46 & 1 & 0 & 0.183504 & -1.956371 & -2.310574 \\
\hline 47 & 1 & 0 & -1.556141 & -1.818030 & -2.655027 \\
\hline 48 & 1 & 0 & -0.759381 & -3.404427 & -2.689258 \\
\hline 49 & 1 & 0 & -0.327898 & -3.778248 & 1.020444 \\
\hline 50 & 1 & 0 & 0.920280 & -3.136354 & -0.055432 \\
\hline 51 & 1 & 0 & -0.063120 & -4.539006 & -0.557384 \\
\hline 52 & 1 & 0 & -1.193911 & -1.886679 & 2.682978 \\
\hline 53 & 1 & 0 & -2.261575 & -3.145498 & 2.030136 \\
\hline 54 & 1 & 0 & -2.939836 & -1.837314 & 3.007050 \\
\hline 55 & 1 & 0 & -4.567560 & -1.217015 & 1.097267 \\
\hline 56 & 1 & 0 & -3.935810 & -2.547912 & 0.131437 \\
\hline 57 & 1 & 0 & -3.943858 & -0.903085 & -0.532613 \\
\hline
\end{tabular}

\section{$2 a$}

\begin{tabular}{|c|c|c|c|c|c|}
\hline \multirow{2}{*}{$\begin{array}{l}\text { Center } \\
\text { Number }\end{array}$} & \multirow{2}{*}{$\begin{array}{l}\text { Atomic } \\
\text { Number }\end{array}$} & \multirow{2}{*}{$\begin{array}{c}\text { Atomic } \\
\text { Type }\end{array}$} & \multicolumn{3}{|c|}{ Coordinates (Angstroms) } \\
\hline & & & $\mathrm{X}$ & $\mathrm{Y}$ & Z \\
\hline & & $-\ldots-\ldots$ & -------- & -------- & --------- \\
\hline 1 & 6 & 0 & 0.665434 & 0.000000 & 0.000000 \\
\hline 2 & 6 & 0 & -0.665434 & 0.000000 & 0.000000 \\
\hline 3 & 1 & 0 & 1.239476 & 0.923262 & 0.000000 \\
\hline 4 & 1 & 0 & 1.239480 & -0.923261 & 0.000000 \\
\hline 5 & 1 & 0 & -1.239479 & -0.923260 & 0.000000 \\
\hline 6 & 1 & 0 & -1.239473 & 0.923263 & 0.000000 \\
\hline
\end{tabular}

\section{$2 \mathrm{~b}$}

\begin{tabular}{|c|c|c|c|c|c|}
\hline Center & Atomic & Atomic & \multicolumn{3}{|c|}{ Coordinates (Angstroms) } \\
\hline Number & Number & Type & $\mathrm{X}$ & $\mathrm{Y}$ & Z \\
\hline & & & & & \\
\hline 1 & 6 & 0 & 2.491769 & -0.013009 & -0.000044 \\
\hline 2 & 6 & 0 & 1.317069 & -0.646419 & 0.000052 \\
\hline 3 & 6 & 0 & 0.043608 & 0.117542 & 0.000017 \\
\hline
\end{tabular}




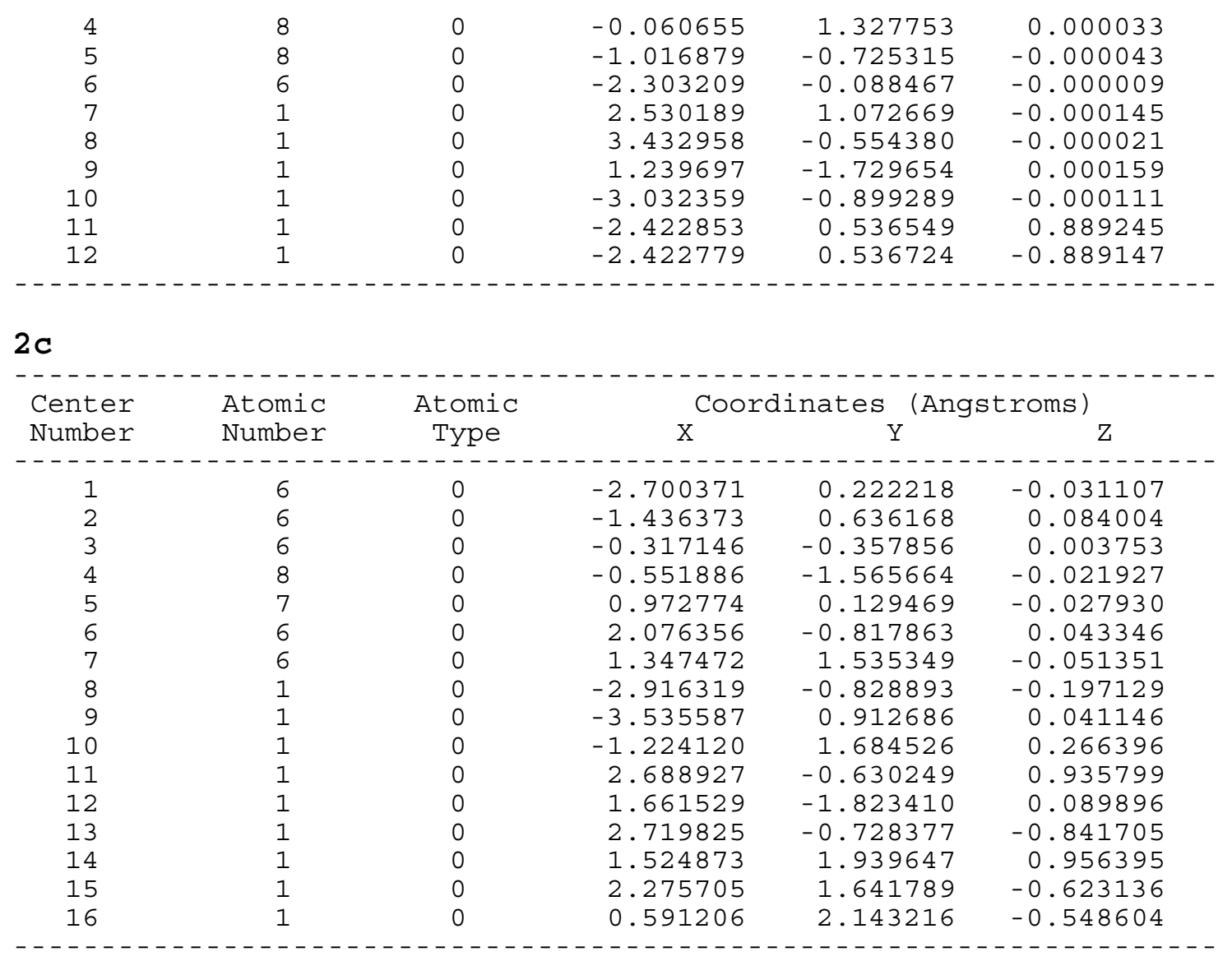

2d

\begin{tabular}{|c|c|c|c|c|c|}
\hline \multirow{2}{*}{$\begin{array}{l}\text { Center } \\
\text { Number }\end{array}$} & \multirow{2}{*}{$\begin{array}{l}\text { Atomic } \\
\text { Number }\end{array}$} & \multirow{2}{*}{$\begin{array}{c}\text { Atomic } \\
\text { Type }\end{array}$} & \multicolumn{3}{|c|}{ Coordinates (Angstroms) } \\
\hline & & & $\mathrm{X}$ & $\mathrm{Y}$ & $\mathrm{Z}$ \\
\hline & & & --------- & -------- & --------- \\
\hline 1 & 6 & 0 & 2.977006 & 0.334991 & 0.000143 \\
\hline 2 & 6 & 0 & 1.954470 & -0.529389 & -0.000107 \\
\hline 3 & 6 & 0 & 0.515104 & -0.220497 & -0.000064 \\
\hline 4 & 6 & 0 & 0.008973 & 1.092279 & -0.000092 \\
\hline 5 & 6 & 0 & -0.406403 & -1.281318 & 0.000001 \\
\hline 6 & 6 & 0 & -1.780771 & -1.046041 & 0.000066 \\
\hline 7 & 6 & 0 & -2.265119 & 0.261886 & 0.000055 \\
\hline 8 & 6 & 0 & -1.361962 & 1.329575 & -0.000028 \\
\hline 9 & 1 & 0 & 2.840188 & 1.413221 & 0.000438 \\
\hline 10 & 1 & 0 & 4.004059 & -0.016942 & 0.000080 \\
\hline 11 & 1 & 0 & 2.186161 & -1.594689 & -0.000349 \\
\hline 12 & 1 & 0 & 0.694229 & 1.935202 & -0.000197 \\
\hline 13 & 1 & 0 & -0.034947 & -2.303788 & 0.000013 \\
\hline 14 & 1 & 0 & -2.472041 & -1.884778 & 0.000121 \\
\hline 15 & 1 & 0 & -3.335271 & 0.450568 & 0.000098 \\
\hline 16 & 1 & 0 & -1.730164 & 2.352295 & -0.000055 \\
\hline
\end{tabular}

\section{$2 e s C s C$}

\begin{tabular}{|c|c|c|c|c|c|}
\hline \multirow{2}{*}{$\begin{array}{l}\text { Center } \\
\text { Number }\end{array}$} & Atomic & Atomic & \multicolumn{3}{|c|}{ Coordinates (Angstroms) } \\
\hline & Number & Type & $\mathrm{X}$ & $\mathrm{Y}$ & Z \\
\hline------ & ----- & ----- & & -------- & \\
\hline $\begin{array}{l}1 \\
2\end{array}$ & 6 & 0 & -0.549059 & -0.381922 & -0.000053 \\
\hline
\end{tabular}




\begin{tabular}{|c|c|c|c|c|c|}
\hline 3 & 6 & 0 & 1.901819 & -0.231223 & 0.000043 \\
\hline 4 & 8 & 0 & 2.136983 & -1.422886 & 0.000037 \\
\hline 5 & 1 & 0 & -0.474291 & -1.464850 & -0.000140 \\
\hline 6 & 6 & 0 & -1.901822 & 0.231236 & -0.000035 \\
\hline 7 & 1 & 0 & 0.474334 & 1.464923 & 0.000140 \\
\hline 8 & 8 & 0 & 2.855106 & 0.725989 & 0.000009 \\
\hline 9 & 6 & 0 & 4.208321 & 0.243174 & -0.000042 \\
\hline 10 & 1 & 0 & 4.836618 & 1.133985 & -0.000057 \\
\hline 11 & 1 & 0 & 4.397840 & -0.363442 & -0.889661 \\
\hline 12 & 1 & 0 & 4.397903 & -0.363459 & 0.889553 \\
\hline 13 & 8 & 0 & -2.137040 & 1.422893 & -0.000012 \\
\hline 14 & 8 & 0 & -2.855076 & -0.726013 & -0.000026 \\
\hline 15 & 6 & 0 & -4.208309 & -0.243218 & 0.000026 \\
\hline 16 & 1 & 0 & -4.397879 & 0.363439 & -0.889549 \\
\hline 17 & 1 & 0 & -4.397843 & 0.363352 & 0.889675 \\
\hline 18 & 1 & 0 & -4.836581 & -1.134049 & 0.000018 \\
\hline \multicolumn{6}{|c|}{ - - - - - - - - - - - - - - - - - - - - - - - - - - - - - - - - - - - - - - - - - - - - - - - - - - - - - - - - - - - - - - } \\
\hline \multicolumn{6}{|c|}{$2 \mathrm{f}$} \\
\hline \multicolumn{6}{|c|}{----------------------------------------------------------} \\
\hline Center & Atomic & Atomic & \multicolumn{3}{|c|}{ Coordinates (Angstroms) } \\
\hline Number & Number & Type & $\mathrm{X}$ & $\mathrm{Y}$ & Z \\
\hline \multicolumn{6}{|c|}{-------------------------------------------------------} \\
\hline 1 & 6 & 0 & 0.685010 & 1.558319 & -0.323010 \\
\hline 2 & 6 & 0 & -0.645296 & 1.420539 & -0.353564 \\
\hline 3 & 6 & 0 & -1.340207 & 0.171913 & 0.047671 \\
\hline 4 & 8 & 0 & -0.824780 & -0.795607 & 0.571214 \\
\hline 5 & 1 & 0 & 1.129588 & 2.519090 & -0.572593 \\
\hline 6 & 6 & 0 & 1.666400 & 0.517566 & 0.128787 \\
\hline 7 & 1 & 0 & -1.277667 & 2.248096 & -0.660038 \\
\hline 8 & 8 & 0 & -2.659930 & 0.258060 & -0.235036 \\
\hline 9 & 6 & 0 & -3.439212 & -0.893830 & 0.124139 \\
\hline 10 & 1 & 0 & -4.463868 & -0.654370 & -0.161032 \\
\hline 11 & 1 & 0 & -3.371458 & -1.082023 & 1.199007 \\
\hline 12 & 1 & 0 & -3.086255 & -1.778034 & -0.413455 \\
\hline 13 & 8 & 0 & 2.316967 & 0.630856 & 1.144149 \\
\hline 14 & 8 & 0 & 1.786128 & -0.483359 & -0.756675 \\
\hline 15 & 6 & 0 & 2.660187 & -1.553043 & -0.354130 \\
\hline 16 & 1 & 0 & 2.253602 & -2.049650 & 0.530448 \\
\hline 17 & 1 & 0 & 3.659792 & -1.172058 & -0.130365 \\
\hline 18 & 1 & 0 & 2.687885 & -2.239437 & -1.200540 \\
\hline
\end{tabular}

\section{TSaa}

\begin{tabular}{|c|c|c|c|c|c|}
\hline \multirow{2}{*}{$\begin{array}{l}\text { Center } \\
\text { Number }\end{array}$} & \multirow{2}{*}{$\begin{array}{l}\text { Atomic } \\
\text { Number }\end{array}$} & \multirow{2}{*}{$\begin{array}{c}\text { Atomic } \\
\text { Type }\end{array}$} & \multicolumn{3}{|c|}{ Coordinates (Angstroms) } \\
\hline & & & $\mathrm{X}$ & $\mathrm{Y}$ & $\mathrm{Z}$ \\
\hline$-\ldots$ & & & --------- & --------- & --------- \\
\hline 1 & 6 & 0 & -1.718018 & -1.201533 & 0.692258 \\
\hline 2 & 6 & 0 & -1.437218 & -1.449068 & -0.603634 \\
\hline 3 & 1 & 0 & -1.215723 & -1.731756 & 1.496046 \\
\hline 4 & 1 & 0 & -2.458222 & -0.461632 & 0.984342 \\
\hline 5 & 1 & 0 & -0.768142 & -2.248571 & -0.900888 \\
\hline 6 & 1 & 0 & -1.964365 & -0.947005 & -1.408125 \\
\hline 7 & 6 & 0 & 0.396875 & 0.155677 & -0.565844 \\
\hline 8 & 15 & 0 & 1.736873 & -0.617028 & 0.149008 \\
\hline 9 & 14 & 0 & -0.450689 & 1.698693 & 0.003221 \\
\hline 10 & 1 & 0 & 2.540598 & 0.193616 & 0.999728 \\
\hline 11 & 1 & 0 & 2.657231 & -0.937666 & -0.874767 \\
\hline 12 & 1 & 0 & -1.859613 & 1.752737 & -0.469455 \\
\hline 13 & 1 & 0 & 0.329384 & 2.762304 & -0.699392 \\
\hline 14 & 1 & 0 & -0.454438 & 2.061240 & 1.455620 \\
\hline
\end{tabular}


TSba

\begin{tabular}{|c|c|c|c|c|c|}
\hline \multirow{2}{*}{$\begin{array}{l}\text { Center } \\
\text { Number }\end{array}$} & \multirow{2}{*}{$\begin{array}{l}\text { Atomic } \\
\text { Number }\end{array}$} & \multirow{2}{*}{$\begin{array}{c}\text { Atomic } \\
\text { Type }\end{array}$} & \multicolumn{3}{|c|}{ Coordinates (Angstroms) } \\
\hline & & & $\mathrm{X}$ & $\mathrm{Y}$ & $\mathrm{Z}$ \\
\hline & & & --------- & --------- & ---------- \\
\hline 1 & 6 & 0 & -0.253089 & 0.041263 & -0.610318 \\
\hline 2 & 14 & 0 & -1.251933 & 1.582245 & -0.396402 \\
\hline 3 & 15 & 0 & 1.158269 & -0.372548 & 0.251618 \\
\hline 4 & 6 & 0 & -1.867789 & -1.701295 & -0.237836 \\
\hline 5 & 6 & 0 & -2.237409 & -1.184537 & 0.953938 \\
\hline 6 & 1 & 0 & -1.708432 & -1.429748 & 1.869970 \\
\hline 7 & 1 & 0 & -3.066760 & -0.487996 & 1.040682 \\
\hline 8 & 1 & 0 & -1.107010 & -2.469497 & -0.314170 \\
\hline 9 & 1 & 0 & -2.417888 & -1.486421 & -1.147705 \\
\hline 10 & 7 & 0 & 1.876657 & 0.955166 & 1.098224 \\
\hline 11 & 7 & 0 & 2.348956 & -0.864494 & -0.927715 \\
\hline 12 & 1 & 0 & -2.626746 & 1.410649 & -0.944954 \\
\hline 13 & 1 & 0 & -0.520145 & 2.548913 & -1.275868 \\
\hline 14 & 1 & 0 & -1.393564 & 2.234535 & 0.938905 \\
\hline 15 & 1 & 0 & 2.702419 & -1.799634 & -0.742031 \\
\hline 16 & 1 & 0 & 1.970593 & -0.845536 & -1.871738 \\
\hline 17 & 1 & 0 & 2.436110 & 0.645710 & 1.888853 \\
\hline 18 & 1 & 0 & 2.454888 & 1.548533 & 0.505144 \\
\hline
\end{tabular}

\section{TSca}

\begin{tabular}{|c|c|c|c|c|c|}
\hline \multirow{2}{*}{$\begin{array}{l}\text { Center } \\
\text { Number }\end{array}$} & \multirow{2}{*}{$\begin{array}{l}\text { Atomic } \\
\text { Number }\end{array}$} & \multirow{2}{*}{$\begin{array}{c}\text { Atomic } \\
\text { Type }\end{array}$} & \multicolumn{3}{|c|}{ Coordinates (Angstroms) } \\
\hline & & & $\mathrm{X}$ & $\mathrm{Y}$ & $\mathrm{Z}$ \\
\hline------ & 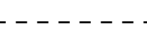 & $2+$ & --------1 & -------- & ----------- \\
\hline 1 & 15 & 0 & -0.453153 & 0.126504 & -0.585460 \\
\hline 2 & 6 & 0 & 1.123188 & -0.376699 & -0.470645 \\
\hline 3 & 14 & 0 & 2.637918 & 0.443686 & -1.126444 \\
\hline 4 & 7 & 0 & -1.702627 & -1.005949 & -0.316020 \\
\hline 5 & 7 & 0 & -1.057513 & 1.435245 & 0.389380 \\
\hline 6 & 6 & 0 & -1.801412 & -2.102909 & -1.273588 \\
\hline 7 & 6 & 0 & -2.136055 & -1.358919 & 1.034443 \\
\hline 8 & 6 & 0 & -0.083772 & 2.418903 & 0.854182 \\
\hline 9 & 6 & 0 & -2.298610 & 2.059325 & -0.079048 \\
\hline 10 & 1 & 0 & 3.828124 & -0.352216 & -0.706112 \\
\hline 11 & 1 & 0 & 2.594708 & 0.448474 & -2.616960 \\
\hline 12 & 1 & 0 & 2.930821 & 1.854795 & -0.714758 \\
\hline 13 & 1 & 0 & -2.836394 & -2.467869 & -1.303692 \\
\hline 14 & 1 & 0 & -1.145120 & -2.949781 & -1.017586 \\
\hline 15 & 1 & 0 & -1.527795 & -1.753012 & -2.272577 \\
\hline 16 & 1 & 0 & -2.026634 & -0.493149 & 1.689679 \\
\hline 17 & 1 & 0 & -1.558704 & -2.200483 & 1.452558 \\
\hline 18 & 1 & 0 & -3.193268 & -1.655490 & 1.017485 \\
\hline 19 & 1 & 0 & -0.536458 & 3.005706 & 1.663272 \\
\hline 20 & 1 & 0 & 0.232977 & 3.120116 & 0.063687 \\
\hline 21 & 1 & 0 & 0.802922 & 1.915169 & 1.241120 \\
\hline 22 & 1 & 0 & -2.991373 & 1.289658 & -0.425259 \\
\hline 23 & 1 & 0 & -2.129439 & 2.778680 & -0.897646 \\
\hline 24 & 1 & 0 & -2.766750 & 2.597974 & 0.754378 \\
\hline 25 & 6 & 0 & 1.766373 & -1.755655 & 1.174137 \\
\hline 26 & 6 & 0 & 2.062367 & -0.739594 & 2.021993 \\
\hline 27 & 1 & 0 & 0.817423 & -2.276984 & 1.228024 \\
\hline 28 & 1 & 0 & 2.536465 & -2.239327 & 0.583334 \\
\hline 29 & 1 & 0 & 1.313356 & -0.314996 & 2.684308 \\
\hline 30 & 1 & 0 & 3.050105 & -0.288203 & 2.046495 \\
\hline
\end{tabular}

cis-TScb 


\begin{tabular}{|c|c|c|c|c|c|}
\hline \multirow{2}{*}{$\begin{array}{l}\text { Center } \\
\text { Number }\end{array}$} & \multirow{2}{*}{$\begin{array}{l}\text { Atomic } \\
\text { Number }\end{array}$} & \multirow{2}{*}{$\begin{array}{c}\text { Atomic } \\
\text { Type }\end{array}$} & \multicolumn{3}{|c|}{ Coordinates (Angstroms) } \\
\hline & & & $\mathrm{X}$ & $\mathrm{Y}$ & Z \\
\hline & & & & & \\
\hline 1 & 15 & 0 & 1.417553 & 0.162194 & 0.133144 \\
\hline 2 & 6 & 0 & 0.624481 & 1.519006 & -0.288619 \\
\hline 3 & 14 & 0 & 0.397343 & 3.040317 & 0.723351 \\
\hline 4 & 1 & 0 & -0.000777 & 2.869579 & 2.156895 \\
\hline 5 & 1 & 0 & -0.669228 & 3.869381 & 0.091928 \\
\hline 6 & 1 & 0 & 1.649344 & 3.851659 & 0.735808 \\
\hline 7 & 6 & 0 & -0.920136 & 1.365820 & -1.818460 \\
\hline 8 & 6 & 0 & -2.031753 & 1.114029 & -1.057973 \\
\hline 9 & 1 & 0 & -0.739557 & 2.355389 & -2.220910 \\
\hline 10 & 1 & 0 & -0.431908 & 0.543734 & -2.327750 \\
\hline 11 & 1 & 0 & -2.646377 & 1.913402 & -0.658344 \\
\hline 12 & 6 & 0 & -2.345830 & -0.249381 & -0.659704 \\
\hline 13 & 8 & 0 & -1.725831 & -1.259477 & -0.974351 \\
\hline 14 & 8 & 0 & -3.440570 & -0.295460 & 0.155686 \\
\hline 15 & 6 & 0 & -3.827974 & -1.603679 & 0.584268 \\
\hline 16 & 1 & 0 & -3.022679 & -2.086166 & 1.146786 \\
\hline 17 & 1 & 0 & -4.701546 & -1.458323 & 1.221927 \\
\hline 18 & 1 & 0 & -4.081656 & -2.237169 & -0.270961 \\
\hline 19 & 7 & 0 & 2.020227 & -0.925555 & -0.994699 \\
\hline 20 & 7 & 0 & 1.104496 & -0.834474 & 1.476129 \\
\hline 21 & 6 & 0 & 2.223009 & -1.502031 & 2.142315 \\
\hline 22 & 1 & 0 & 1.870393 & -2.439058 & 2.590904 \\
\hline 23 & 1 & 0 & 3.001340 & -1.741512 & 1.414708 \\
\hline 24 & 1 & 0 & 2.660019 & -0.881092 & 2.939896 \\
\hline 25 & 6 & 0 & -0.007855 & -0.504254 & 2.362408 \\
\hline 26 & 1 & 0 & 0.280214 & 0.200750 & 3.156695 \\
\hline 27 & 1 & 0 & -0.827119 & -0.066843 & 1.790611 \\
\hline 28 & 1 & 0 & -0.364668 & -1.427644 & 2.836022 \\
\hline 29 & 6 & 0 & 2.837462 & -0.406326 & -2.088109 \\
\hline 30 & 1 & 0 & 2.246190 & -0.246326 & -3.001180 \\
\hline 31 & 1 & 0 & 3.288772 & 0.546207 & -1.801680 \\
\hline 32 & 1 & 0 & 3.637668 & -1.122201 & -2.317518 \\
\hline 33 & 6 & 0 & 1.405204 & -2.230439 & -1.254386 \\
\hline 34 & 1 & 0 & 0.809505 & -2.534557 & -0.394956 \\
\hline 35 & 1 & 0 & 0.743316 & -2.188170 & -2.129126 \\
\hline 36 & 1 & 0 & 2.191155 & -2.975182 & -1.434975 \\
\hline
\end{tabular}

\section{trans-TScb}

\begin{tabular}{|c|c|c|c|c|c|}
\hline \multirow{2}{*}{$\begin{array}{l}\text { Center } \\
\text { Number }\end{array}$} & \multirow{2}{*}{$\begin{array}{l}\text { Atomic } \\
\text { Number }\end{array}$} & \multirow{2}{*}{$\begin{array}{l}\text { Atomic } \\
\text { Type }\end{array}$} & \multicolumn{3}{|c|}{ Coordinates (Angstroms) } \\
\hline & & & $\mathrm{X}$ & $\mathrm{Y}$ & $\mathrm{Z}$ \\
\hline & & & --------- & --------- & --------- \\
\hline 1 & 15 & 0 & -1.654881 & -0.093363 & 0.459779 \\
\hline 2 & 6 & 0 & -0.343427 & -1.009328 & 0.800489 \\
\hline 3 & 14 & 0 & 0.658164 & -1.070964 & 2.358684 \\
\hline 4 & 1 & 0 & 1.530310 & 0.114439 & 2.611769 \\
\hline 5 & 1 & 0 & 1.524930 & -2.277666 & 2.364735 \\
\hline 6 & 1 & 0 & -0.268741 & -1.184499 & 3.523028 \\
\hline 7 & 7 & 0 & -2.694495 & -0.498050 & -0.816607 \\
\hline 8 & 7 & 0 & -1.623531 & 1.619677 & 0.309398 \\
\hline 9 & 6 & 0 & -2.862894 & 2.347685 & 0.595586 \\
\hline 10 & 1 & 0 & -2.846385 & 3.308896 & 0.067449 \\
\hline 11 & 1 & 0 & -3.720526 & 1.773290 & 0.238824 \\
\hline 12 & 1 & 0 & -2.992068 & 2.549150 & 1.670854 \\
\hline 13 & 6 & 0 & -0.421337 & 2.321035 & 0.756046 \\
\hline 14 & 1 & 0 & -0.375015 & 2.438217 & 1.850484 \\
\hline 15 & 1 & 0 & 0.470174 & 1.786214 & 0.425461 \\
\hline 16 & 1 & 0 & -0.416364 & 3.321798 & 0.307220 \\
\hline 17 & 6 & 0 & -3.329686 & -1.812239 & -0.773866 \\
\hline 18 & 1 & 0 & -2.729630 & -2.584370 & -1.278966 \\
\hline
\end{tabular}




\begin{tabular}{|c|c|c|c|c|c|}
\hline 19 & 1 & 0 & -3.479584 & -2.123159 & 0.262837 \\
\hline 20 & 1 & 0 & -4.307849 & -1.759667 & -1.269250 \\
\hline 21 & 6 & 0 & -2.517746 & 0.040955 & -2.164650 \\
\hline 22 & 1 & 0 & -2.053397 & 1.026331 & -2.106501 \\
\hline 23 & 1 & 0 & -1.889863 & -0.614875 & -2.789178 \\
\hline 24 & 1 & 0 & -3.494987 & 0.140735 & -2.654383 \\
\hline 25 & 6 & 0 & 0.858078 & -1.806415 & -0.891419 \\
\hline 26 & 6 & 0 & 1.516107 & -0.678361 & -1.291058 \\
\hline 27 & 6 & 0 & 2.784997 & -0.281751 & -0.680911 \\
\hline 28 & 8 & 0 & 3.392777 & -0.886878 & 0.187733 \\
\hline 29 & 8 & 0 & 3.234017 & 0.893896 & -1.208785 \\
\hline 30 & 6 & 0 & 4.486052 & 1.352461 & -0.685201 \\
\hline 31 & 1 & 0 & 1.359960 & -2.522354 & -0.249790 \\
\hline 32 & 1 & 0 & -0.001882 & -2.169665 & -1.440108 \\
\hline 33 & 1 & 0 & 1.096436 & -0.010165 & -2.036742 \\
\hline 34 & 1 & 0 & 5.279513 & 0.626063 & -0.883995 \\
\hline 35 & 1 & 0 & 4.693750 & 2.292741 & -1.198344 \\
\hline 36 & 1 & 0 & 4.421108 & 1.510700 & 0.395130 \\
\hline
\end{tabular}

\section{cis-TS' cb}

\begin{tabular}{|c|c|c|c|c|c|}
\hline \multirow{2}{*}{$\begin{array}{l}\text { Center } \\
\text { Number }\end{array}$} & \multirow{2}{*}{$\begin{array}{l}\text { Atomic } \\
\text { Number }\end{array}$} & \multirow{2}{*}{$\begin{array}{c}\text { Atomic } \\
\text { Type }\end{array}$} & \multicolumn{3}{|c|}{ Coordinates (Angstroms) } \\
\hline & & & $\mathrm{X}$ & $\mathrm{Y}$ & Z \\
\hline & & & ----------1 & --------- & ---------- \\
\hline 1 & 15 & 0 & 1.387728 & 0.217403 & -0.058901 \\
\hline 2 & 6 & 0 & 0.466446 & 1.456268 & -0.591045 \\
\hline 3 & 14 & 0 & 0.422730 & 3.166945 & 0.099469 \\
\hline 4 & 1 & 0 & 0.276471 & 3.316227 & 1.581022 \\
\hline 5 & 1 & 0 & -0.729690 & 3.888667 & -0.510975 \\
\hline 6 & 1 & 0 & 1.665643 & 3.902192 & -0.273891 \\
\hline 7 & 6 & 0 & -1.342338 & 1.070045 & -1.745534 \\
\hline 8 & 6 & 0 & -2.279555 & 1.067834 & -0.747038 \\
\hline 9 & 1 & 0 & -1.209022 & 1.950140 & -2.363467 \\
\hline 10 & 1 & 0 & -0.991726 & 0.137252 & -2.169026 \\
\hline 11 & 1 & 0 & -2.740717 & 1.990142 & -0.409460 \\
\hline 12 & 6 & 0 & -2.673106 & -0.107640 & 0.021775 \\
\hline 13 & 8 & 0 & -3.427661 & -0.093126 & 0.981591 \\
\hline 14 & 8 & 0 & -2.111698 & -1.271272 & -0.439117 \\
\hline 15 & 6 & 0 & -2.518790 & -2.448462 & 0.266205 \\
\hline 16 & 1 & 0 & -3.605412 & -2.566264 & 0.231068 \\
\hline 17 & 1 & 0 & -2.029479 & -3.281802 & -0.240884 \\
\hline 18 & 1 & 0 & -2.208023 & -2.405708 & 1.314537 \\
\hline 19 & 7 & 0 & 1.757799 & -1.100879 & -1.043136 \\
\hline 20 & 7 & 0 & 1.313724 & -0.510847 & 1.487507 \\
\hline 21 & 6 & 0 & 2.565756 & -1.019937 & 2.055533 \\
\hline 22 & 1 & 0 & 2.336474 & -1.807056 & 2.783856 \\
\hline 23 & 1 & 0 & 3.184435 & -1.451153 & 1.265362 \\
\hline 24 & 1 & 0 & 3.142007 & -0.233696 & 2.567890 \\
\hline 25 & 6 & 0 & 0.409060 & 0.067101 & 2.482059 \\
\hline 26 & 1 & 0 & 0.825561 & 0.962853 & 2.967087 \\
\hline 27 & 1 & 0 & -0.543978 & 0.331741 & 2.020998 \\
\hline 28 & 1 & 0 & 0.222628 & -0.685187 & 3.257931 \\
\hline 29 & 6 & 0 & 2.297254 & -0.828589 & -2.372703 \\
\hline 30 & 1 & 0 & 1.512119 & -0.829649 & -3.143076 \\
\hline 31 & 1 & 0 & 2.786719 & 0.147701 & -2.387326 \\
\hline 32 & 1 & 0 & 3.035430 & -1.598449 & -2.632935 \\
\hline 33 & 6 & 0 & 1.120165 & -2.409660 & -0.904954 \\
\hline 34 & 1 & 0 & 0.772674 & -2.541074 & 0.119856 \\
\hline 35 & 1 & 0 & 0.262014 & -2.510921 & -1.585285 \\
\hline 36 & 1 & 0 & 1.846581 & -3.198709 & -1.138506 \\
\hline
\end{tabular}

\section{trans-TS' $\mathrm{cb}$}




\begin{tabular}{|c|c|c|c|c|c|}
\hline \multirow{2}{*}{$\begin{array}{l}\text { Center } \\
\text { Number }\end{array}$} & \multirow{2}{*}{$\begin{array}{l}\text { Atomic } \\
\text { Number }\end{array}$} & \multirow{2}{*}{$\begin{array}{l}\text { Atomic } \\
\text { Type }\end{array}$} & \multicolumn{3}{|c|}{ Coordinates (Angstroms) } \\
\hline & & & $\mathrm{X}$ & $\mathrm{Y}$ & $\mathrm{Z}$ \\
\hline & & & 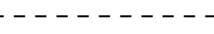 & --------- & ---------- \\
\hline 1 & 15 & 0 & -1.585722 & 0.011057 & 0.569399 \\
\hline 2 & 6 & 0 & -0.157889 & -0.675345 & 0.991269 \\
\hline 3 & 14 & 0 & 0.985053 & -0.186244 & 2.360747 \\
\hline 4 & 1 & 0 & 1.739545 & 1.091692 & 2.173618 \\
\hline 5 & 1 & 0 & 1.999718 & -1.252514 & 2.585551 \\
\hline 6 & 1 & 0 & 0.208061 & -0.044490 & 3.625731 \\
\hline 7 & 7 & 0 & -2.708849 & -0.849591 & -0.366028 \\
\hline 8 & 7 & 0 & -1.714930 & 1.572223 & -0.136618 \\
\hline 9 & 6 & 0 & -2.974375 & 2.296908 & 0.056081 \\
\hline 10 & 1 & 0 & -3.103783 & 3.017845 & -0.760231 \\
\hline 11 & 1 & 0 & -3.812277 & 1.596727 & 0.033256 \\
\hline 12 & 1 & 0 & -2.998344 & 2.849272 & 1.008793 \\
\hline 13 & 6 & 0 & -0.537061 & 2.439969 & -0.119174 \\
\hline 14 & 1 & 0 & -0.363279 & 2.905292 & 0.864086 \\
\hline 15 & 1 & 0 & 0.354768 & 1.881189 & -0.405846 \\
\hline 16 & 1 & 0 & -0.690143 & 3.242240 & -0.850768 \\
\hline 17 & 6 & 0 & -3.202070 & -2.112065 & 0.176354 \\
\hline 18 & 1 & 0 & -2.589163 & -2.970306 & -0.139159 \\
\hline 19 & 1 & 0 & -3.200352 & -2.077328 & 1.268503 \\
\hline 20 & 1 & 0 & -4.230816 & -2.280328 & -0.167591 \\
\hline 21 & 6 & 0 & -2.731986 & -0.773135 & -1.826459 \\
\hline 22 & 1 & 0 & -2.355419 & 0.198828 & -2.147911 \\
\hline 23 & 1 & 0 & -2.119454 & -1.565072 & -2.287509 \\
\hline 24 & 1 & 0 & -3.762059 & -0.889267 & -2.187477 \\
\hline 25 & 6 & 0 & 0.939151 & -1.849090 & -0.530606 \\
\hline 26 & 6 & 0 & 1.352056 & -0.830224 & -1.342232 \\
\hline 27 & 6 & 0 & 2.570412 & -0.039897 & -1.162754 \\
\hline 28 & 8 & 0 & 2.844875 & 0.952601 & -1.819357 \\
\hline 29 & 8 & 0 & 3.379551 & -0.516049 & -0.176544 \\
\hline 30 & 6 & 0 & 4.579853 & 0.235952 & 0.043517 \\
\hline 31 & 1 & 0 & 1.610294 & -2.281310 & 0.202572 \\
\hline 32 & 1 & 0 & 0.084143 & -2.454573 & -0.806554 \\
\hline 33 & 1 & 0 & 0.723438 & -0.476489 & -2.153874 \\
\hline 34 & 1 & 0 & 4.345907 & 1.270130 & 0.309811 \\
\hline 35 & 1 & 0 & 5.091247 & -0.264047 & 0.866932 \\
\hline 36 & 1 & 0 & 5.205551 & 0.234793 & -0.853618 \\
\hline
\end{tabular}

\section{cis-TScc}

\begin{tabular}{|c|c|c|c|c|c|}
\hline \multirow{2}{*}{$\begin{array}{l}\text { Center } \\
\text { Number }\end{array}$} & \multirow{2}{*}{$\begin{array}{l}\text { Atomic } \\
\text { Number }\end{array}$} & \multirow{2}{*}{$\begin{array}{c}\text { Atomic } \\
\text { Type }\end{array}$} & \multicolumn{3}{|c|}{ Coordinates (Angstroms) } \\
\hline & & & $\mathrm{X}$ & $\mathrm{Y}$ & Z \\
\hline------1 & 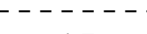 & & --------- & --------- & ---------- \\
\hline 1 & 15 & 0 & 1.664498 & 0.191535 & 0.055619 \\
\hline 2 & 6 & 0 & 0.806541 & 1.523867 & -0.294248 \\
\hline 3 & 14 & 0 & 0.562461 & 3.012593 & 0.753965 \\
\hline 4 & 1 & 0 & 0.285360 & 2.804966 & 2.211675 \\
\hline 5 & 1 & 0 & -0.601949 & 3.779261 & 0.221674 \\
\hline 6 & 1 & 0 & 1.754097 & 3.909669 & 0.690126 \\
\hline 7 & 6 & 0 & -0.740829 & 1.262175 & -1.745102 \\
\hline 8 & 6 & 0 & -1.872477 & 1.006731 & -1.006078 \\
\hline 9 & 1 & 0 & -0.607996 & 2.232888 & -2.209016 \\
\hline 10 & 1 & 0 & -0.231314 & 0.435449 & -2.224797 \\
\hline 11 & 1 & 0 & -2.503160 & 1.831205 & -0.692487 \\
\hline 12 & 6 & 0 & -2.116506 & -0.345658 & -0.501759 \\
\hline 13 & 8 & 0 & -1.225125 & -1.218197 & -0.515308 \\
\hline 14 & 7 & 0 & -3.368310 & -0.637772 & 0.013454 \\
\hline 15 & 6 & 0 & -4.495259 & 0.277501 & -0.012229 \\
\hline 16 & 6 & 0 & -3.588974 & -1.914199 & 0.669718 \\
\hline 17 & 1 & 0 & -4.423331 & 0.955736 & -0.863775 \\
\hline
\end{tabular}




\begin{tabular}{|c|c|c|c|c|c|}
\hline 18 & 1 & 0 & -4.579520 & 0.873115 & 0.909997 \\
\hline 19 & 7 & 0 & 2.234156 & -0.844293 & -1.132305 \\
\hline 20 & 7 & 0 & 1.628370 & -0.795342 & 1.435493 \\
\hline 21 & 6 & 0 & 2.899804 & -1.218895 & 2.023635 \\
\hline 22 & 1 & 0 & 2.759184 & -2.178639 & 2.535905 \\
\hline 23 & 1 & 0 & 3.645138 & -1.356815 & 1.236666 \\
\hline 24 & 1 & 0 & 3.286890 & -0.491339 & 2.754180 \\
\hline 25 & 6 & 0 & 0.535810 & -0.625928 & 2.390707 \\
\hline 26 & 1 & 0 & 0.683889 & 0.230428 & 3.065188 \\
\hline 27 & 1 & 0 & -0.400829 & -0.504800 & 1.846021 \\
\hline 28 & 1 & 0 & 0.468658 & -1.535397 & 3.000543 \\
\hline 29 & 6 & 0 & 2.867399 & -0.278272 & -2.319156 \\
\hline 30 & 1 & 0 & 2.171935 & -0.232010 & -3.169558 \\
\hline 31 & 1 & 0 & 3.220848 & 0.733868 & -2.111212 \\
\hline 32 & 1 & 0 & 3.723512 & -0.901250 & -2.611248 \\
\hline 33 & 6 & 0 & 1.755217 & -2.220085 & -1.286165 \\
\hline 34 & 1 & 0 & 1.266577 & -2.544001 & -0.368776 \\
\hline 35 & 1 & 0 & 1.026762 & -2.291246 & -2.104014 \\
\hline 36 & 1 & 0 & 2.603555 & -2.882335 & -1.504220 \\
\hline 37 & 1 & 0 & -5.420945 & -0.298977 & -0.124019 \\
\hline 38 & 1 & 0 & -2.670204 & -2.494939 & 0.600861 \\
\hline 39 & 1 & 0 & -4.404717 & -2.466520 & 0.183252 \\
\hline 40 & 1 & 0 & -3.857254 & -1.773643 & 1.727256 \\
\hline
\end{tabular}

\section{trans-TScC}

\begin{tabular}{|c|c|c|c|c|c|}
\hline \multirow{2}{*}{$\begin{array}{l}\text { Center } \\
\text { Number }\end{array}$} & Atomic & Atomic & \multicolumn{3}{|c|}{ Coordinates (Angstroms) } \\
\hline & Number & Type & $\mathrm{X}$ & $\mathrm{Y}$ & Z \\
\hline & & & -------- & -------- & -------- \\
\hline 1 & 15 & 0 & 1.905290 & 0.341988 & 0.276156 \\
\hline 2 & 6 & 0 & 0.690159 & 1.375012 & -0.095214 \\
\hline 3 & 14 & 0 & -0.159944 & 2.629097 & 0.973869 \\
\hline 4 & 1 & 0 & -1.072552 & 2.085211 & 2.021750 \\
\hline 5 & 1 & 0 & -0.921685 & 3.597852 & 0.145759 \\
\hline 6 & 1 & 0 & 0.898553 & 3.405184 & 1.688525 \\
\hline 7 & 7 & 0 & 2.827254 & -0.389409 & -0.951987 \\
\hline 8 & 7 & 0 & 1.716948 & -1.001951 & 1.345375 \\
\hline 9 & 6 & 0 & 2.927692 & -1.522056 & 1.987087 \\
\hline 10 & 1 & 0 & 2.756334 & -2.562468 & 2.290156 \\
\hline 11 & 1 & 0 & 3.759047 & -1.500931 & 1.279553 \\
\hline 12 & 1 & 0 & 3.208871 & -0.946772 & 2.883879 \\
\hline 13 & 6 & 0 & 0.559294 & -0.988147 & 2.237543 \\
\hline 14 & 1 & 0 & 0.696025 & -0.325825 & 3.107760 \\
\hline 15 & 1 & 0 & -0.329938 & -0.663419 & 1.695272 \\
\hline 16 & 1 & 0 & 0.394511 & -2.006400 & 2.611443 \\
\hline 17 & 6 & 0 & 3.571757 & 0.500341 & -1.838586 \\
\hline 18 & 1 & 0 & 2.975815 & 0.829615 & -2.703859 \\
\hline 19 & 1 & 0 & 3.894743 & 1.389651 & -1.291734 \\
\hline 20 & 1 & 0 & 4.461587 & -0.021867 & -2.213543 \\
\hline 21 & 6 & 0 & 2.412948 & -1.644012 & -1.578005 \\
\hline 22 & 1 & 0 & 1.880493 & -2.257033 & -0.849031 \\
\hline 23 & 1 & 0 & 1.760202 & -1.475859 & -2.450729 \\
\hline 24 & 1 & 0 & 3.298061 & -2.195658 & -1.920942 \\
\hline 25 & 6 & 0 & -0.617831 & 0.885088 & -1.844901 \\
\hline 26 & 6 & 0 & -1.356698 & -0.134712 & -1.319751 \\
\hline 27 & 6 & 0 & -2.584778 & 0.159944 & -0.553678 \\
\hline 28 & 8 & 0 & -2.915012 & 1. 324649 & -0.301354 \\
\hline 29 & 7 & 0 & -3.347929 & -0.908216 & -0.106994 \\
\hline 30 & 6 & 0 & -4.624903 & -0.612605 & 0.529103 \\
\hline 31 & 1 & 0 & -1.023014 & 1.890306 & -1.856770 \\
\hline 32 & 1 & 0 & 0.208945 & 0.682293 & -2.514250 \\
\hline 33 & 1 & 0 & -1.012509 & -1.155769 & -1.440138 \\
\hline 34 & 6 & 0 & -3.152645 & -2.286844 & -0.532047 \\
\hline
\end{tabular}




$\begin{array}{rrrrrr}35 & 1 & 0 & -5.466525 & -0.775374 & -0.160643 \\ 36 & 1 & 0 & -4.619801 & 0.431696 & 0.836863 \\ 37 & 1 & 0 & -4.765029 & -1.259449 & 1.403278 \\ 38 & 1 & 0 & -3.526178 & -2.476165 & -1.549680 \\ 39 & 1 & 0 & -3.699098 & -2.941248 & 0.153566 \\ 40 & 1 & 0 & -2.100765 & -2.576987 & -0.486231 \\ - & & & \end{array}$

\section{cis-TScd}

\begin{tabular}{|c|c|c|c|c|c|}
\hline Center & Atomic & Atomic & \multicolumn{3}{|c|}{ Coordinates (Angstroms) } \\
\hline Number & Number & Type & $\mathrm{X}$ & $\mathrm{Y}$ & $\mathrm{Z}$ \\
\hline------ & & & -------- & -------- & -------- \\
\hline 1 & 15 & 0 & -1.899384 & -0.009022 & 0.255957 \\
\hline 2 & 6 & 0 & -1.251632 & 1.408562 & -0.275353 \\
\hline 3 & 14 & 0 & -1.177645 & 2.999428 & 0.647949 \\
\hline 4 & 7 & 0 & -2.381043 & -1.201091 & -0.858678 \\
\hline 5 & 7 & 0 & -1.197244 & -1.009945 & 1.474927 \\
\hline 6 & 6 & 0 & -3.362283 & -0.806066 & -1.864182 \\
\hline 7 & 6 & 0 & -1.490477 & -2.277396 & -1.285246 \\
\hline 8 & 1 & 0 & -3.960184 & -1.680216 & -2.153692 \\
\hline 9 & 1 & 0 & -0.783580 & -2.504971 & -0.486459 \\
\hline 10 & 6 & 0 & 0.278089 & 1.483733 & -1.865805 \\
\hline 11 & 6 & 0 & 1.424584 & 1.612295 & -1.129031 \\
\hline 12 & 1 & 0 & -0.005424 & 0.540696 & -2.320096 \\
\hline 13 & 1 & 0 & -0.183375 & 2.361722 & -2.299789 \\
\hline 14 & 1 & 0 & 1.706074 & 2.615357 & -0.811422 \\
\hline 15 & 6 & 0 & -0.137936 & -0.426770 & 2.293776 \\
\hline 16 & 6 & 0 & -2.110705 & -1.868999 & 2.232372 \\
\hline 17 & 1 & 0 & 0.389514 & -1.238992 & 2.808910 \\
\hline 18 & 1 & 0 & -2.870903 & -2.280786 & 1.564779 \\
\hline 19 & 1 & 0 & -0.551697 & 4.034831 & -0.227299 \\
\hline 20 & 1 & 0 & -2.554229 & 3.465637 & 0.983393 \\
\hline 21 & 1 & 0 & -2.891442 & -0.394692 & -2.770503 \\
\hline 22 & 1 & 0 & -4.032242 & -0.045376 & -1.455875 \\
\hline 23 & 1 & 0 & -0.516871 & 0.271105 & 3.057965 \\
\hline 24 & 1 & 0 & 0.575111 & 0.103284 & 1.660579 \\
\hline 25 & 1 & 0 & -0.928329 & -2.008620 & -2.195629 \\
\hline 26 & 1 & 0 & -2.076394 & -3.179079 & -1.507706 \\
\hline 27 & 1 & 0 & -0.402820 & 3.042541 & 1.930490 \\
\hline 28 & 1 & 0 & -2.614255 & -1.333379 & 3.053782 \\
\hline 29 & 1 & 0 & -1.544284 & -2.702340 & 2.666353 \\
\hline 30 & 6 & 0 & 2.307845 & 0.548414 & -0.666154 \\
\hline 31 & 6 & 0 & 3.345321 & 0.861804 & 0.237105 \\
\hline 32 & 6 & 0 & 4.226237 & -0.114028 & 0.697095 \\
\hline 33 & 6 & 0 & 4.096698 & -1.435865 & 0.266409 \\
\hline 34 & 6 & 0 & 3.077498 & -1.766513 & -0.632767 \\
\hline 35 & 6 & 0 & 2.199528 & -0.791856 & -1.095496 \\
\hline 36 & 1 & 0 & 3.452728 & 1.889065 & 0.578082 \\
\hline 37 & 1 & 0 & 5.016039 & 0.158350 & 1.392465 \\
\hline 38 & 1 & 0 & 4.784080 & -2.199111 & 0.620683 \\
\hline 39 & 1 & 0 & 2.973950 & -2.790853 & -0.982021 \\
\hline 40 & 1 & 0 & 1.427733 & -1.067482 & -1.807692 \\
\hline
\end{tabular}

\section{trans-TScd}

\begin{tabular}{cccccc} 
Center & Atomic & Atomic & \multicolumn{2}{c}{ Coordinates } & (Angstroms) \\
Number & Number & Type & $\mathrm{X}$ & $\mathrm{Y}$ & $\mathrm{Z}$ \\
-1 & 15 & 0 & 2.107692 & -0.163849 & 0.511484 \\
1 & 6 & 0 & 0.803050 & -1.170093 & 0.560812 \\
3 & 14 & 0 & -0.254165 & -1.599940 & 2.006705 \\
4 & 7 & 0 & 3.209824 & -0.287431 & -0.787253
\end{tabular}




\begin{tabular}{|c|c|c|c|c|c|}
\hline 5 & 7 & 0 & 1.938652 & 1.563782 & 0.567622 \\
\hline 6 & 6 & 0 & 3.921861 & -1.555539 & -0.911814 \\
\hline 7 & 6 & 0 & 2.962143 & 0.379400 & -2.064424 \\
\hline 8 & 1 & 0 & 4.876425 & -1.387455 & -1.427326 \\
\hline 9 & 1 & 0 & 2.442505 & 1.322597 & -1.888058 \\
\hline 10 & 6 & 0 & -0.400814 & -1.527431 & -1.316511 \\
\hline 11 & 6 & 0 & -1.083417 & -0.346635 & -1.387845 \\
\hline 12 & 1 & 0 & 0.504112 & -1.664834 & -1.893544 \\
\hline 13 & 1 & 0 & -0.859374 & -2.439224 & -0.948857 \\
\hline 14 & 1 & 0 & -0.577379 & 0.492451 & -1.864574 \\
\hline 15 & 6 & 0 & 0.724011 & 2.087073 & 1.187471 \\
\hline 16 & 6 & 0 & 3.134248 & 2.325839 & 0.939180 \\
\hline 17 & 1 & 0 & 0.620559 & 3.145111 & 0.915578 \\
\hline 18 & 1 & 0 & 4.014088 & 1.885986 & 0.465400 \\
\hline 19 & 1 & 0 & -1.187302 & -2.703505 & 1.635502 \\
\hline 20 & 1 & 0 & 0.605434 & -2.111662 & 3.112611 \\
\hline 21 & 1 & 0 & 3.351690 & -2.308400 & -1.478681 \\
\hline 22 & 1 & 0 & 4.130505 & -1.965953 & 0.079687 \\
\hline 23 & 1 & 0 & 0.738586 & 2.021426 & 2.288227 \\
\hline 24 & 1 & 0 & -0.150330 & 1.545415 & 0.824048 \\
\hline 25 & 1 & 0 & 2.358459 & -0.239392 & -2.749461 \\
\hline 26 & 1 & 0 & 3.918257 & 0.592639 & -2.560483 \\
\hline 27 & 1 & 0 & -1.112699 & -0.520647 & 2.589942 \\
\hline 28 & 1 & 0 & 3.295635 & 2.355313 & 2.029430 \\
\hline 29 & 1 & 0 & 3.028192 & 3.358611 & 0.584068 \\
\hline 30 & 6 & 0 & -2.414717 & -0.040319 & -0.873939 \\
\hline 31 & 6 & 0 & -2.887414 & 1.285540 & -0.958716 \\
\hline 32 & 6 & 0 & -4.156659 & 1.632902 & -0.502464 \\
\hline 33 & 6 & 0 & -4.991161 & 0.661098 & 0.052093 \\
\hline 34 & 6 & 0 & -4.541919 & -0.660622 & 0.141410 \\
\hline 35 & 6 & 0 & -3.275601 & -1.009037 & -0.315943 \\
\hline 36 & 1 & 0 & -2.243154 & 2.047164 & -1.392640 \\
\hline 37 & 1 & 0 & -4.494702 & 2.662969 & -0.580392 \\
\hline 38 & 1 & 0 & -5.981987 & 0.927583 & 0.409482 \\
\hline 39 & 1 & 0 & -5.185830 & -1.424976 & 0.568474 \\
\hline 40 & 1 & 0 & -2.949757 & -2.041836 & -0.243018 \\
\hline
\end{tabular}

cis-TSce-sc-sc

\begin{tabular}{|c|c|c|c|c|c|}
\hline \multirow{2}{*}{$\begin{array}{l}\text { Center } \\
\text { Number }\end{array}$} & \multirow{2}{*}{$\begin{array}{l}\text { Atomic } \\
\text { Number }\end{array}$} & \multirow{2}{*}{$\begin{array}{l}\text { Atomic } \\
\text { Type }\end{array}$} & \multicolumn{3}{|c|}{ Coordinates (Angstroms) } \\
\hline & & & $\mathrm{X}$ & $\mathrm{Y}$ & $\mathrm{Z}$ \\
\hline & & & --------- & & --------- \\
\hline 1 & 15 & 0 & -0.840812 & -1.442452 & 0.331924 \\
\hline 2 & 6 & 0 & 0.322745 & -0.504348 & 0.985605 \\
\hline 3 & 14 & 0 & 0.481156 & -0.051114 & 2.773713 \\
\hline 4 & 1 & 0 & -0.793917 & -0.142989 & 3.552075 \\
\hline 5 & 1 & 0 & 0.956933 & 1.350061 & 2.885543 \\
\hline 6 & 1 & 0 & 1.453228 & -0.948145 & 3.460531 \\
\hline 7 & 6 & 0 & 1.303577 & 0.764178 & -0.396216 \\
\hline 8 & 6 & 0 & 0.843857 & 2.031319 & -0.109014 \\
\hline 9 & 6 & 0 & 2.695777 & 0.384821 & -0.027404 \\
\hline 10 & 1 & 0 & 0.889080 & 0.247274 & -1.252617 \\
\hline 11 & 1 & 0 & 1.384797 & 2.677437 & 0.571485 \\
\hline 12 & 6 & 0 & -0.412769 & 2.488228 & -0.667593 \\
\hline 13 & 8 & 0 & -1.211730 & 1.811638 & -1.313751 \\
\hline 14 & 8 & 0 & -0.638960 & 3.803247 & -0.387697 \\
\hline 15 & 6 & 0 & -1.854072 & 4.343168 & -0.912346 \\
\hline 16 & 1 & 0 & -2.725726 & 3.836495 & -0.485837 \\
\hline 17 & 1 & 0 & -1.854672 & 5.396862 & -0.628684 \\
\hline 18 & 1 & 0 & -1.890548 & 4.241083 & -2.001013 \\
\hline 19 & 7 & 0 & -0.632967 & -2.168107 & -1.162913 \\
\hline 20 & 7 & 0 & -2.520864 & -1.254528 & 0.441488 \\
\hline 21 & 6 & 0 & -3.348861 & -2.447638 & 0.630996 \\
\hline
\end{tabular}




\begin{tabular}{|c|c|c|c|c|c|}
\hline 22 & 1 & 0 & -4.328213 & -2.285788 & 0.164768 \\
\hline 23 & 1 & 0 & -2.878083 & -3.307715 & 0.148591 \\
\hline 24 & 1 & 0 & -3.505519 & -2.680473 & 1.695017 \\
\hline 25 & 6 & 0 & -3.069183 & -0.022266 & 1.009553 \\
\hline 26 & 1 & 0 & -3.041411 & -0.008193 & 2.108338 \\
\hline 27 & 1 & 0 & -2.517430 & 0.832922 & 0.615477 \\
\hline 28 & 1 & 0 & -4.115406 & 0.063036 & 0.690612 \\
\hline 29 & 6 & 0 & 0.585189 & -2.937909 & -1.414489 \\
\hline 30 & 1 & 0 & 1.345703 & -2.341269 & -1.933984 \\
\hline 31 & 1 & 0 & 1.013367 & -3.278493 & -0.468934 \\
\hline 32 & 1 & 0 & 0.338490 & -3.814322 & -2.027978 \\
\hline 33 & 6 & 0 & -1.375441 & -1.769245 & -2.360910 \\
\hline 34 & 1 & 0 & -2.242230 & -1.173361 & -2.076960 \\
\hline 35 & 1 & 0 & -0.743866 & -1.170053 & -3.030975 \\
\hline 36 & 1 & 0 & -1.712969 & -2.661385 & -2.904320 \\
\hline 37 & 8 & 0 & 3.350763 & 0.869454 & 0.872260 \\
\hline 38 & 8 & 0 & 3.166355 & -0.584423 & -0.851460 \\
\hline 39 & 6 & 0 & 4.495778 & -1.038043 & -0.557321 \\
\hline 40 & 1 & 0 & 4.722297 & -1.792898 & -1.311459 \\
\hline 41 & 1 & 0 & 4.540054 & -1.471490 & 0.445804 \\
\hline 42 & 1 & 0 & 5.207863 & -0.210685 & -0.616336 \\
\hline
\end{tabular}

cis-TSce-sc-st

\begin{tabular}{|c|c|c|c|c|c|}
\hline \multirow{2}{*}{$\begin{array}{l}\text { Center } \\
\text { Number }\end{array}$} & \multirow{2}{*}{$\begin{array}{l}\text { Atomic } \\
\text { Number }\end{array}$} & \multirow{2}{*}{$\begin{array}{c}\text { Atomic } \\
\text { Type }\end{array}$} & \multicolumn{3}{|c|}{ Coordinates (Angstroms) } \\
\hline & & & $\mathrm{X}$ & $\mathrm{Y}$ & $\mathrm{Z}$ \\
\hline & & & -------- & -------- & -------- \\
\hline 1 & 15 & 0 & -0.934944 & -1.312872 & 0.376422 \\
\hline 2 & 6 & 0 & 0.370684 & -0.522071 & 0.970898 \\
\hline 3 & 14 & 0 & 0.756165 & -0.293855 & 2.772162 \\
\hline 4 & 1 & 0 & -0.442865 & -0.263067 & 3.666253 \\
\hline 5 & 1 & 0 & 1.475468 & 0.988050 & 2.963500 \\
\hline 6 & 1 & 0 & 1.612719 & -1.411279 & 3.260861 \\
\hline 7 & 6 & 0 & 1.379068 & 0.781915 & -0.368609 \\
\hline 8 & 6 & 0 & 1.049520 & 2.031020 & 0.107411 \\
\hline 9 & 6 & 0 & 2.742017 & 0.231022 & -0.122951 \\
\hline 10 & 1 & 0 & 0.879750 & 0.404685 & -1.251325 \\
\hline 11 & 1 & 0 & 1.664366 & 2.509162 & 0.860444 \\
\hline 12 & 6 & 0 & -0.124312 & 2.780467 & -0.313683 \\
\hline 13 & 8 & 0 & -0.417344 & 3.900256 & 0.071801 \\
\hline 14 & 8 & 0 & -0.902179 & 2.101768 & -1.223449 \\
\hline 15 & 6 & 0 & -2.021571 & 2.843363 & -1.719226 \\
\hline 16 & 1 & 0 & -1.691566 & 3.759900 & -2.216813 \\
\hline 17 & 1 & 0 & -2.521375 & 2.184341 & -2.431506 \\
\hline 18 & 1 & 0 & -2.703074 & 3.115724 & -0.908050 \\
\hline 19 & 7 & 0 & -0.952579 & -1.905619 & -1.196673 \\
\hline 20 & 7 & 0 & -2.566851 & -0.880132 & 0.621392 \\
\hline 21 & 6 & 0 & -3.562827 & -1.952883 & 0.691403 \\
\hline 22 & 1 & 0 & -4.535446 & -1.562990 & 0.367533 \\
\hline 23 & 1 & 0 & -3.280087 & -2.768892 & 0.022526 \\
\hline 24 & 1 & 0 & -3.673586 & -2.351514 & 1.711428 \\
\hline 25 & 6 & 0 & -2.873570 & 0.271345 & 1.469392 \\
\hline 26 & 1 & 0 & -2.885925 & 0.019172 & 2.539532 \\
\hline 27 & 1 & 0 & -2.138806 & 1.060532 & 1.304365 \\
\hline 28 & 1 & 0 & -3.865576 & 0.650741 & 1.194763 \\
\hline 29 & 6 & 0 & 0.152889 & -2.766788 & -1.619321 \\
\hline 30 & 1 & 0 & 0.964709 & -2.195955 & -2.088794 \\
\hline 31 & 1 & 0 & 0.566053 & -3.293563 & -0.755945 \\
\hline 32 & 1 & 0 & -0.222621 & -3.507737 & -2.336414 \\
\hline 33 & 6 & 0 & -1.688549 & -1.267635 & -2.288895 \\
\hline 34 & 1 & 0 & -2.502646 & -0.668204 & -1.880810 \\
\hline 35 & 1 & 0 & -1.034422 & -0.616929 & -2.886896 \\
\hline 36 & 1 & 0 & -2.109378 & -2.037289 & -2.948340 \\
\hline
\end{tabular}




$\begin{array}{rrrrrr}37 & 8 & 0 & 3.494463 & 0.564815 & 0.768662 \\ 38 & 8 & 0 & 3.059402 & -0.699771 & -1.056516 \\ 39 & 6 & 0 & 4.350460 & -1.305850 & -0.892296 \\ 40 & 1 & 0 & 4.458843 & -1.997723 & -1.728455 \\ 41 & 1 & 0 & 4.403901 & -1.841833 & 0.059515 \\ 42 & 1 & 0 & 5.137844 & -0.548002 & -0.915722 \\ -\end{array}$

cis-TSce-st-sc

\begin{tabular}{|c|c|c|c|c|c|}
\hline Center & Atomic & Atomic & \multicolumn{3}{|c|}{ Coordinates (Angstroms) } \\
\hline Number & Number & Type & $\mathrm{X}$ & $\mathrm{Y}$ & Z \\
\hline & & & 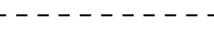 & -------- & ---------- \\
\hline 1 & 15 & 0 & -1.173944 & -1.202107 & 0.378365 \\
\hline 2 & 6 & 0 & 0.278779 & -0.617966 & 0.860657 \\
\hline 3 & 14 & 0 & 0.847501 & -0.430981 & 2.612683 \\
\hline 4 & 1 & 0 & -0.209803 & -0.039457 & 3.595943 \\
\hline 5 & 1 & 0 & 1.899641 & 0.613700 & 2.675052 \\
\hline 6 & 1 & 0 & 1.422237 & -1.714847 & 3.108819 \\
\hline 7 & 6 & 0 & 1.345582 & 0.455497 & -0.606524 \\
\hline 8 & 6 & 0 & 1.456883 & 1.732008 & -0.094748 \\
\hline 9 & 6 & 0 & 2.505364 & -0.474273 & -0.717358 \\
\hline 10 & 1 & 0 & 0.612410 & 0.276032 & -1.382273 \\
\hline 11 & 1 & 0 & 2.300938 & 2.015246 & 0.521386 \\
\hline 12 & 6 & 0 & 0.470966 & 2.780930 & -0.306291 \\
\hline 13 & 8 & 0 & 0.584852 & 3.935165 & 0.072634 \\
\hline 14 & 8 & 0 & -0.636156 & 2.352980 & -1.002592 \\
\hline 15 & 6 & 0 & -1.579822 & 3.384439 & -1.311066 \\
\hline 16 & 1 & 0 & -1.119326 & 4.161311 & -1.928506 \\
\hline 17 & 1 & 0 & -2.386096 & 2.893471 & -1.859151 \\
\hline 18 & 1 & 0 & -1.965396 & 3.849606 & -0.399310 \\
\hline 19 & 7 & 0 & -1.382047 & -1.816220 & -1.168025 \\
\hline 20 & 7 & 0 & -2.695107 & -0.509521 & 0.712591 \\
\hline 21 & 6 & 0 & -3.843021 & -1.404896 & 0.880159 \\
\hline 22 & 1 & 0 & -4.759731 & -0.872531 & 0.599229 \\
\hline 23 & 1 & 0 & -3.736125 & -2.273395 & 0.226342 \\
\hline 24 & 1 & 0 & -3.950591 & -1.753561 & 1.918596 \\
\hline 25 & 6 & 0 & -2.751114 & 0.698155 & 1.535725 \\
\hline 26 & 1 & 0 & -2.690157 & 0.483720 & 2.612416 \\
\hline 27 & 1 & 0 & -1.934601 & 1.367459 & 1.260953 \\
\hline 28 & 1 & 0 & -3.704013 & 1.205568 & 1.341203 \\
\hline 29 & 6 & 0 & -0.464255 & -2.861864 & -1.631284 \\
\hline 30 & 1 & 0 & 0.385534 & -2.444288 & -2.184273 \\
\hline 31 & 1 & 0 & -0.069078 & -3.414019 & -0.775598 \\
\hline 32 & 1 & 0 & -1.013446 & -3.559346 & -2.276268 \\
\hline 33 & 6 & 0 & -2.106552 & -1.116737 & -2.229776 \\
\hline 34 & 1 & 0 & -2.743448 & -0.345650 & -1.795538 \\
\hline 35 & 1 & 0 & -1.410486 & -0.646572 & -2.939232 \\
\hline 36 & 1 & 0 & -2.734080 & -1.826816 & -2.783731 \\
\hline 37 & 8 & 0 & 2.608276 & -1.323312 & -1.582436 \\
\hline 38 & 8 & 0 & 3.444645 & -0.262040 & 0.228417 \\
\hline 39 & 6 & 0 & 4.593193 & -1.121007 & 0.157187 \\
\hline 40 & 1 & 0 & 5.239179 & -0.811923 & 0.979253 \\
\hline 41 & 1 & 0 & 5.104399 & -0.999233 & -0.801487 \\
\hline 42 & 1 & 0 & 4.297307 & -2.167004 & 0.272927 \\
\hline
\end{tabular}

\section{cis-TSce-st-st}

\begin{tabular}{|c|c|c|c|c|c|}
\hline Center & Atomic & Atomic & \multicolumn{3}{|c|}{ Coordinates (Angstroms) } \\
\hline Number & Number & Type & $\mathrm{X}$ & $\mathrm{Y}$ & Z \\
\hline----- & 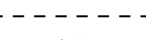 & & & --------- & ---------1 \\
\hline 1 & 15 & 0 & -1.173944 & -1.202107 & 0.378365 \\
\hline 2 & 6 & 0 & 0.278779 & -0.617966 & 0.860657 \\
\hline
\end{tabular}




\begin{tabular}{|c|c|c|c|c|c|}
\hline 3 & 14 & 0 & 0.847501 & -0.430981 & 2.612683 \\
\hline 4 & 1 & 0 & -0.209803 & -0.039457 & 3.595943 \\
\hline 5 & 1 & 0 & 1.899641 & 0.613700 & 2.675052 \\
\hline 6 & 1 & 0 & 1.422237 & -1.714847 & 3.108819 \\
\hline 7 & 6 & 0 & 1.345582 & 0.455497 & -0.606524 \\
\hline 8 & 6 & 0 & 1.456883 & 1.732008 & -0.094748 \\
\hline 9 & 6 & 0 & 2.505364 & -0.474273 & -0.717358 \\
\hline 10 & 1 & 0 & 0.612410 & 0.276032 & -1.382273 \\
\hline 11 & 1 & 0 & 2.300938 & 2.015246 & 0.521386 \\
\hline 12 & 6 & 0 & 0.470966 & 2.780930 & -0.306291 \\
\hline 13 & 8 & 0 & 0.584852 & 3.935165 & 0.072634 \\
\hline 14 & 8 & 0 & -0.636156 & 2.352980 & -1.002592 \\
\hline 15 & 6 & 0 & -1.579822 & 3.384439 & -1.311066 \\
\hline 16 & 1 & 0 & -1.119326 & 4.161311 & -1.928506 \\
\hline 17 & 1 & 0 & -2.386096 & 2.893471 & -1.859151 \\
\hline 18 & 1 & 0 & -1.965396 & 3.849606 & -0.399310 \\
\hline 19 & 7 & 0 & -1.382047 & -1.816220 & -1.168025 \\
\hline 20 & 7 & 0 & -2.695107 & -0.509521 & 0.712591 \\
\hline 21 & 6 & 0 & -3.843021 & -1.404896 & 0.880159 \\
\hline 22 & 1 & 0 & -4.759731 & -0.872531 & 0.599229 \\
\hline 23 & 1 & 0 & -3.736125 & -2.273395 & 0.226342 \\
\hline 24 & 1 & 0 & -3.950591 & -1.753561 & 1.918596 \\
\hline 25 & 6 & 0 & -2.751114 & 0.698155 & 1.535725 \\
\hline 26 & 1 & 0 & -2.690157 & 0.483720 & 2.612416 \\
\hline 27 & 1 & 0 & -1.934601 & 1.367459 & 1.260953 \\
\hline 28 & 1 & 0 & -3.704013 & 1.205568 & 1.341203 \\
\hline 29 & 6 & 0 & -0.464255 & -2.861864 & -1.631284 \\
\hline 30 & 1 & 0 & 0.385534 & -2.444288 & -2.184273 \\
\hline 31 & 1 & 0 & -0.069078 & -3.414019 & -0.775598 \\
\hline 32 & 1 & 0 & -1.013446 & -3.559346 & -2.276268 \\
\hline 33 & 6 & 0 & -2.106552 & -1.116737 & -2.229776 \\
\hline 34 & 1 & 0 & -2.743448 & -0.345650 & -1.795538 \\
\hline 35 & 1 & 0 & -1.410486 & -0.646572 & -2.939232 \\
\hline 36 & 1 & 0 & -2.734080 & -1.826816 & -2.783731 \\
\hline 37 & 8 & 0 & 2.608276 & -1.323312 & -1.582436 \\
\hline 38 & 8 & 0 & 3.444645 & -0.262040 & 0.228417 \\
\hline 39 & 6 & 0 & 4.593193 & -1.121007 & 0.157187 \\
\hline 40 & 1 & 0 & 5.239179 & -0.811923 & 0.979253 \\
\hline 41 & 1 & 0 & 5.104399 & -0.999233 & -0.801487 \\
\hline 42 & 1 & 0 & 4.297307 & -2.167004 & 0.272927 \\
\hline
\end{tabular}

\section{trans-TSce-sc-sc}

\begin{tabular}{|c|c|c|c|c|c|}
\hline \multirow{2}{*}{$\begin{array}{l}\text { Center } \\
\text { Number }\end{array}$} & Atomic & \multirow{2}{*}{$\begin{array}{c}\text { Atomic } \\
\text { Type }\end{array}$} & \multicolumn{3}{|c|}{ Coordinates (Angstroms) } \\
\hline & Number & & $\mathrm{X}$ & $\mathrm{Y}$ & $\mathrm{Z}$ \\
\hline------- & ----- & ---- & -------- & -------- & -------- \\
\hline 1 & 15 & 0 & 1.045278 & 1.318677 & 0.489276 \\
\hline 2 & 6 & 0 & 0.220689 & 0.044989 & 1.128743 \\
\hline 3 & 14 & 0 & -0.803478 & 0.168892 & 2.682019 \\
\hline 4 & 1 & 0 & -2.049034 & 0.982280 & 2.564338 \\
\hline 5 & 1 & 0 & -1.188772 & -1.194305 & 3.129342 \\
\hline 6 & 1 & 0 & 0.013673 & 0.779435 & 3.770534 \\
\hline 7 & 7 & 0 & 2.342020 & 1.155023 & -0.564274 \\
\hline 8 & 7 & 0 & 0.265225 & 2.702611 & -0.143678 \\
\hline 9 & 6 & 0 & 0.965882 & 3.985702 & -0.054640 \\
\hline 10 & 1 & 0 & 0.645756 & 4.631511 & -0.881413 \\
\hline 11 & 1 & 0 & 2.043164 & 3.827922 & -0.139831 \\
\hline 12 & 1 & 0 & 0.756649 & 4.508543 & 0.891561 \\
\hline 13 & 6 & 0 & -1.188528 & 2.817675 & -0.058449 \\
\hline 14 & 1 & 0 & -1.531003 & 3.190603 & 0.919131 \\
\hline 15 & 1 & 0 & -1.653716 & 1.849613 & -0.246862 \\
\hline 16 & 1 & 0 & -1.526384 & 3.522040 & -0.828618 \\
\hline 17 & 6 & 0 & 2.216578 & 1.265014 & -2.020460 \\
\hline
\end{tabular}




$\begin{array}{rrrrrr}18 & 1 & 0 & 3.096235 & 1.781227 & -2.426538 \\ 19 & 1 & 0 & 1.326767 & 1.845111 & -2.268465 \\ 20 & 1 & 0 & 2.139664 & 0.268748 & -2.472242 \\ 21 & 6 & 0 & 3.535217 & 0.441667 & -0.114996 \\ 22 & 1 & 0 & 3.551297 & 0.385599 & 0.976369 \\ 23 & 1 & 0 & 4.432794 & 0.978028 & -0.451554 \\ 24 & 1 & 0 & 3.559879 & -0.576080 & -0.522896 \\ 25 & 6 & 0 & -0.390071 & -1.671578 & 0.075269 \\ 26 & 6 & 0 & -1.276363 & -1.157747 & -0.845593 \\ 27 & 6 & 0 & -2.669583 & -0.940586 & -0.482616 \\ 28 & 8 & 0 & -3.188032 & -1.202650 & 0.593998 \\ 29 & 8 & 0 & -3.367948 & -0.382149 & -1.514692 \\ 30 & 6 & 0 & -4.760369 & -0.168656 & -1.255992 \\ 31 & 1 & 0 & -0.794035 & -2.136533 & 0.967451 \\ 32 & 6 & 0 & 0.927835 & -2.199376 & -0.372340 \\ 33 & 1 & 0 & -0.941523 & -0.863061 & -1.833933 \\ 34 & 1 & 0 & -5.266496 & -1.115988 & -1.047998 \\ 35 & 1 & 0 & -5.162178 & 0.286097 & -2.162784 \\ 36 & 1 & 0 & -4.900589 & 0.495888 & -0.398481 \\ 37 & 8 & 0 & 1.521118 & -1.881582 & -1.386759 \\ 38 & 8 & 0 & 1.376754 & -3.136871 & 0.488839 \\ 39 & 6 & 0 & 2.624277 & -3.754613 & 0.144953 \\ 40 & 1 & 0 & 2.766467 & -4.552546 & 0.874419 \\ 41 & 1 & 0 & 2.588966 & -4.159553 & -0.869714 \\ 42 & 1 & 0 & 3.442707 & -3.031599 & 0.212085 \\ ------------------1\end{array}$

\section{trans-TSce-sc-st}

\begin{tabular}{|c|c|c|c|c|c|}
\hline \multirow{2}{*}{$\begin{array}{l}\text { Center } \\
\text { Number }\end{array}$} & \multirow{2}{*}{$\begin{array}{l}\text { Atomic } \\
\text { Number }\end{array}$} & \multirow{2}{*}{$\begin{array}{c}\text { Atomic } \\
\text { Type }\end{array}$} & \multicolumn{3}{|c|}{ Coordinates (Angstroms) } \\
\hline & & & $\mathrm{X}$ & $\mathrm{Y}$ & Z \\
\hline & & & --------- & -------- & -------- \\
\hline 1 & 15 & 0 & -1.196849 & -1.129282 & 0.566337 \\
\hline 2 & 6 & 0 & -0.049917 & -0.034487 & 1.025694 \\
\hline 3 & 14 & 0 & 1.139617 & -0.358424 & 2.419744 \\
\hline 4 & 1 & 0 & 2.169869 & -1.412683 & 2.167714 \\
\hline 5 & 1 & 0 & 1.873641 & 0.895257 & 2.741447 \\
\hline 6 & 1 & 0 & 0.399168 & -0.766766 & 3.646910 \\
\hline 7 & 7 & 0 & -2.583611 & -0.705446 & -0.280060 \\
\hline 8 & 7 & 0 & -0.795631 & -2.624651 & -0.158744 \\
\hline 9 & 6 & 0 & -1.700296 & -3.754192 & 0.070275 \\
\hline 10 & 1 & 0 & -1.645327 & -4.440281 & -0.783509 \\
\hline 11 & 1 & 0 & -2.728495 & -3.396360 & 0.157437 \\
\hline 12 & 1 & 0 & -1.441478 & -4.316112 & 0.981100 \\
\hline 13 & 6 & 0 & 0.606680 & -3.013103 & -0.301855 \\
\hline 14 & 1 & 0 & 1.040493 & -3.397883 & 0.633969 \\
\hline 15 & 1 & 0 & 1.204538 & -2.170326 & -0.649309 \\
\hline 16 & 1 & 0 & 0.667049 & -3.806881 & -1.055813 \\
\hline 17 & 6 & 0 & -2.717630 & -0.832890 & -1.733817 \\
\hline 18 & 1 & 0 & -3.726344 & -1.189150 & -1.980853 \\
\hline 19 & 1 & 0 & -1.991322 & -1.556102 & -2.106791 \\
\hline 20 & 1 & 0 & -2.547931 & 0.136538 & -2.217757 \\
\hline 21 & 6 & 0 & -3.538343 & 0.213548 & 0.334446 \\
\hline 22 & 1 & 0 & -3.368940 & 0.267060 & 1.412913 \\
\hline 23 & 1 & 0 & -4.561371 & -0.145927 & 0.158505 \\
\hline 24 & 1 & 0 & -3.441443 & 1.220378 & -0.089918 \\
\hline 25 & 6 & 0 & 0.709739 & 1.511380 & -0.157653 \\
\hline 26 & 6 & 0 & 1.275966 & 0.810620 & -1.201170 \\
\hline 27 & 6 & 0 & 2.609928 & 0.226995 & -1.176179 \\
\hline 28 & 8 & 0 & 3.046837 & -0.532784 & -2.028753 \\
\hline 29 & 8 & 0 & 3.342958 & 0.603574 & -0.087304 \\
\hline 30 & 6 & 0 & 4.664119 & 0.052692 & -0.023357 \\
\hline 31 & 1 & 0 & 1.350134 & 1.889929 & 0.630052 \\
\hline 32 & 6 & 0 & -0.529488 & 2.306500 & -0.386079 \\
\hline
\end{tabular}




$\begin{array}{rrrrrr}33 & 1 & 0 & 0.686650 & 0.577930 & -2.081451 \\ 34 & 1 & 0 & 4.629563 & -1.039898 & 0.004149 \\ 35 & 1 & 0 & 5.099958 & 0.443645 & 0.896881 \\ 36 & 1 & 0 & 5.254272 & 0.364588 & -0.889763 \\ 37 & 8 & 0 & -1.341078 & 2.129896 & -1.275354 \\ 38 & 8 & 0 & -0.620528 & 3.304990 & 0.518084 \\ 39 & 6 & 0 & -1.760600 & 4.164996 & 0.385673 \\ 40 & 1 & 0 & -1.609965 & 4.964454 & 1.111803 \\ 41 & 1 & 0 & -1.824724 & 4.569571 & -0.627673 \\ 42 & 1 & 0 & -2.681656 & 3.619105 & 0.610846\end{array}$

\section{trans-TSce-st-sc}

\begin{tabular}{|c|c|c|c|c|c|}
\hline \multirow{2}{*}{$\begin{array}{l}\text { Center } \\
\text { Number }\end{array}$} & \multirow{2}{*}{$\begin{array}{l}\text { Atomic } \\
\text { Number }\end{array}$} & \multirow{2}{*}{$\begin{array}{c}\text { Atomic } \\
\text { Type }\end{array}$} & \multicolumn{3}{|c|}{ Coordinates (Angstroms) } \\
\hline & & & $\mathrm{X}$ & $\mathrm{Y}$ & $\mathrm{Z}$ \\
\hline 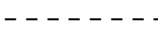 & 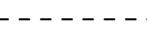 & & --------- & -------- & --------- \\
\hline 1 & 15 & 0 & 1.158577 & 1.256253 & 0.463831 \\
\hline 2 & 6 & 0 & 0.114272 & 0.237783 & 1.224975 \\
\hline 3 & 14 & 0 & -1.047170 & 0.828556 & 2.563031 \\
\hline 4 & 1 & 0 & -2.149112 & 1.721698 & 2.099154 \\
\hline 5 & 1 & 0 & -1.652719 & -0.340508 & 3.248485 \\
\hline 6 & 1 & 0 & -0.270375 & 1.591443 & 3.582368 \\
\hline 7 & 7 & 0 & 2.561755 & 0.734177 & -0.304304 \\
\hline 8 & 7 & 0 & 0.653186 & 2.522113 & -0.572254 \\
\hline 9 & 6 & 0 & 1.470633 & 3.738117 & -0.606763 \\
\hline 10 & 1 & 0 & 1.352484 & 4.226001 & -1.581963 \\
\hline 11 & 1 & 0 & 2.524632 & 3.482737 & -0.475958 \\
\hline 12 & 1 & 0 & 1.179977 & 4.456785 & 0.175044 \\
\hline 13 & 6 & 0 & -0.775550 & 2.772853 & -0.752506 \\
\hline 14 & 1 & 0 & -1.210888 & 3.365947 & 0.066290 \\
\hline 15 & 1 & 0 & -1.317496 & 1.829577 & -0.827698 \\
\hline 16 & 1 & 0 & -0.913899 & 3.329413 & -1.687612 \\
\hline 17 & 6 & 0 & 2.681326 & 0.563563 & -1.751249 \\
\hline 18 & 1 & 0 & 3.681880 & 0.874165 & -2.079069 \\
\hline 19 & 1 & 0 & 1.939507 & 1.184878 & -2.254559 \\
\hline 20 & 1 & 0 & 2.523223 & -0.484135 & -2.040327 \\
\hline 21 & 6 & 0 & 3.558919 & 0.020260 & 0.488390 \\
\hline 22 & 1 & 0 & 3.431605 & 0.252372 & 1.548299 \\
\hline 23 & 1 & 0 & 4.565558 & 0.330165 & 0.177856 \\
\hline 24 & 1 & 0 & 3.477440 & -1.067880 & 0.366918 \\
\hline 25 & 6 & 0 & -0.617996 & -1.616178 & 0.512464 \\
\hline 26 & 6 & 0 & -1.369425 & -1.214721 & -0.570873 \\
\hline 27 & 6 & 0 & -2.736671 & -0.742814 & -0.391556 \\
\hline 28 & 8 & 0 & -3.354807 & -0.687212 & 0.661692 \\
\hline 29 & 8 & 0 & -3.277796 & -0.345408 & -1.580944 \\
\hline 30 & 6 & 0 & -4.638078 & 0.098289 & -1.507826 \\
\hline 31 & 1 & 0 & -1.131975 & -1.798165 & 1.449523 \\
\hline 32 & 6 & 0 & 0.598850 & -2.465718 & 0.371899 \\
\hline 33 & 1 & 0 & -0.950075 & -1.197021 & -1.569805 \\
\hline 34 & 1 & 0 & -5.289372 & -0.704769 & -1.150594 \\
\hline 35 & 1 & 0 & -4.909595 & 0.384180 & -2.525182 \\
\hline 36 & 1 & 0 & -4.733844 & 0.951601 & -0.830270 \\
\hline 37 & 8 & 0 & 1.016713 & -3.197119 & 1.243625 \\
\hline 38 & 8 & 0 & 1.158401 & -2.399898 & -0.865660 \\
\hline 39 & 6 & 0 & 2.199992 & -3.359083 & -1.105539 \\
\hline 40 & 1 & 0 & 2.516712 & -3.197369 & -2.136741 \\
\hline 41 & 1 & 0 & 3.037469 & -3.210121 & -0.419093 \\
\hline 42 & 1 & 0 & 1.818297 & -4.375607 & -0.977255 \\
\hline
\end{tabular}

\section{trans-TSce-st-st}

Center Atomic Atomic Coordinates (Angstroms)




\begin{tabular}{|c|c|c|c|c|c|}
\hline Number & Number & Type & $\mathrm{X}$ & $\mathrm{Y}$ & $\mathrm{Z}$ \\
\hline & & & & & \\
\hline 1 & 15 & 0 & -1.124536 & -1.225432 & 0.549210 \\
\hline 2 & 6 & 0 & 0.064926 & -0.227686 & 1.108953 \\
\hline 3 & 14 & 0 & 1.373226 & -0.825041 & 2.292997 \\
\hline 4 & 1 & 0 & 2.381173 & -1.773438 & 1.726738 \\
\hline 5 & 1 & 0 & 2.125847 & 0.340072 & 2.830021 \\
\hline 6 & 1 & 0 & 0.737048 & -1.516038 & 3.449398 \\
\hline 7 & 7 & 0 & -2.602993 & -0.646119 & -0.010879 \\
\hline 8 & 7 & 0 & -0.825411 & -2.504977 & -0.547955 \\
\hline 9 & 6 & 0 & -1.700908 & -3.677917 & -0.468170 \\
\hline 10 & 1 & 0 & -1.749151 & -4.159989 & -1.452027 \\
\hline 11 & 1 & 0 & -2.710511 & -3.371046 & -0.185965 \\
\hline 12 & 1 & 0 & -1.338411 & -4.419025 & 0.260928 \\
\hline 13 & 6 & 0 & 0.549640 & -2.826610 & -0.928932 \\
\hline 14 & 1 & 0 & 1.078722 & -3.412398 & -0.161417 \\
\hline 15 & 1 & 0 & 1.115943 & -1.917765 & -1.133276 \\
\hline 16 & 1 & 0 & 0.519711 & -3.422573 & -1.848910 \\
\hline 17 & 6 & 0 & -2.924197 & -0.465918 & -1.425891 \\
\hline 18 & 1 & 0 & -3.970114 & -0.746052 & -1.606851 \\
\hline 19 & 1 & 0 & -2.280459 & -1.105471 & -2.031048 \\
\hline 20 & 1 & 0 & -2.777935 & 0.577964 & -1.733177 \\
\hline 21 & 6 & 0 & -3.448114 & 0.100572 & 0.915997 \\
\hline 22 & 1 & 0 & -3.189627 & -0.152484 & 1.947033 \\
\hline 23 & 1 & 0 & -4.500886 & -0.159540 & 0.743626 \\
\hline 24 & 1 & 0 & -3.334324 & 1.185746 & 0.793198 \\
\hline 25 & 6 & 0 & 0.740498 & 1.563010 & 0.240748 \\
\hline 26 & 6 & 0 & 1.238446 & 1.090760 & -0.955829 \\
\hline 27 & 6 & 0 & 2.559983 & 0.498886 & -1.128773 \\
\hline 28 & 8 & 0 & 2.924049 & -0.080252 & -2.142349 \\
\hline 29 & 8 & 0 & 3.365569 & 0.646653 & -0.039302 \\
\hline 30 & 6 & 0 & 4.675800 & 0.079494 & -0.167400 \\
\hline 31 & 1 & 0 & 1.428745 & 1.744590 & 1.057681 \\
\hline 32 & 6 & 0 & -0.433957 & 2.481094 & 0.311656 \\
\hline 33 & 1 & 0 & 0.610583 & 1.054517 & -1.838480 \\
\hline 34 & 1 & 0 & 4.616844 & -0.996622 & -0.351168 \\
\hline 35 & 1 & 0 & 5.174255 & 0.277959 & 0.782047 \\
\hline 36 & 1 & 0 & 5.219290 & 0.549660 & -0.991765 \\
\hline 37 & 8 & 0 & -0.633043 & 3.247584 & 1.229622 \\
\hline 38 & 8 & 0 & -1.222512 & 2.432305 & -0.793263 \\
\hline 39 & 6 & 0 & -2.239921 & 3.444819 & -0.850732 \\
\hline 40 & 1 & 0 & -2.758008 & 3.287305 & -1.797471 \\
\hline 41 & 1 & 0 & -2.934852 & 3.351110 & -0.012264 \\
\hline 42 & 1 & 0 & -1.787858 & 4.439887 & -0.822541 \\
\hline
\end{tabular}

cis-TSCf-sc-sc

\begin{tabular}{|c|c|c|c|c|c|}
\hline \multirow{2}{*}{$\begin{array}{l}\text { Center } \\
\text { Number }\end{array}$} & \multirow{2}{*}{$\begin{array}{l}\text { Atomic } \\
\text { Number }\end{array}$} & \multirow{2}{*}{$\begin{array}{c}\text { Atomic } \\
\text { Type }\end{array}$} & \multicolumn{3}{|c|}{ Coordinates (Angstroms) } \\
\hline & & & $\mathrm{X}$ & $\mathrm{Y}$ & $\mathrm{Z}$ \\
\hline 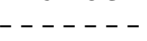 & & & -------- & ------- & -------- \\
\hline 1 & 15 & 0 & 0.609600 & -1.078157 & -0.009361 \\
\hline 2 & 6 & 0 & -0.431306 & -0.893433 & 1.203193 \\
\hline 3 & 14 & 0 & -0.186615 & -1.288163 & 2.969160 \\
\hline 4 & 1 & 0 & 1.161092 & -1.837056 & 3.328897 \\
\hline 5 & 1 & 0 & -0.387926 & -0.077460 & 3.821161 \\
\hline 6 & 1 & 0 & -1.178796 & -2.304424 & 3.429901 \\
\hline 7 & 7 & 0 & 0.187719 & -1.522485 & -1.552767 \\
\hline 8 & 7 & 0 & 2.274552 & -1.261491 & 0.041164 \\
\hline 9 & 6 & 0 & 2.988419 & -2.355252 & -0.609957 \\
\hline 10 & 1 & 0 & 3.817027 & -1.964208 & -1.216110 \\
\hline 11 & 1 & 0 & 2.310192 & -2.907103 & -1.261546 \\
\hline 12 & 1 & 0 & 3.404895 & -3.046173 & 0.137456 \\
\hline 13 & 6 & 0 & 3.077477 & -0.448138 & 0.946477 \\
\hline
\end{tabular}




\begin{tabular}{|c|c|c|c|c|c|}
\hline 14 & 1 & 0 & 3.511076 & -1.060985 & 1.748190 \\
\hline 15 & 1 & 0 & 2.461438 & 0.329410 & 1.400653 \\
\hline 16 & 1 & 0 & 3.895453 & 0.033082 & 0.392844 \\
\hline 17 & 6 & 0 & 0.748257 & -0.854317 & -2.732022 \\
\hline 18 & 1 & 0 & 0.918923 & -1.599539 & -3.519349 \\
\hline 19 & 1 & 0 & 1.692910 & -0.373465 & -2.480515 \\
\hline 20 & 1 & 0 & 0.057478 & -0.085707 & -3.095898 \\
\hline 21 & 6 & 0 & -1.089360 & -2.194719 & -1.798554 \\
\hline 22 & 1 & 0 & -1.458530 & -2.638184 & -0.872284 \\
\hline 23 & 1 & 0 & -0.937458 & -2.987274 & -2.543208 \\
\hline 24 & 1 & 0 & -1.833994 & -1.484554 & -2.173755 \\
\hline 25 & 6 & 0 & -1.478604 & 1.082210 & 1.078851 \\
\hline 26 & 6 & 0 & -0.505133 & 2.063813 & 1.072814 \\
\hline 27 & 6 & 0 & 0.494551 & 2.216568 & 0.053368 \\
\hline 28 & 8 & 0 & 0.816816 & 1.371394 & -0.794926 \\
\hline 29 & 8 & 0 & 1.181417 & 3.387058 & 0.166068 \\
\hline 30 & 6 & 0 & 2.253411 & 3.573736 & -0.761138 \\
\hline 31 & 1 & 0 & -2.038435 & 0.979216 & 2.003100 \\
\hline 32 & 6 & 0 & -2.304334 & 0.677425 & -0.097182 \\
\hline 33 & 1 & 0 & -0.335933 & 2.625540 & 1.985983 \\
\hline 34 & 1 & 0 & 1.890448 & 3.534503 & -1.792420 \\
\hline 35 & 1 & 0 & 3.025200 & 2.808145 & -0.632331 \\
\hline 36 & 1 & 0 & 2.662658 & 4.560979 & -0.540762 \\
\hline 37 & 8 & 0 & -2.056765 & 0.823878 & -1.277083 \\
\hline 38 & 8 & 0 & -3.484332 & 0.172424 & 0.351108 \\
\hline 39 & 6 & 0 & -4.427511 & -0.195921 & -0.659703 \\
\hline 40 & 1 & 0 & -5.352716 & -0.425650 & -0.129185 \\
\hline 41 & 1 & 0 & -4.581329 & 0.623516 & -1.366926 \\
\hline 42 & 1 & 0 & -4.083242 & -1.078014 & -1.208717 \\
\hline
\end{tabular}

cis-TScf-sc-st

\begin{tabular}{|c|c|c|c|c|c|}
\hline Center & Atomic & Atomic & \multicolumn{3}{|c|}{ Coordinates (Angstroms) } \\
\hline Number & Number & Type & $\mathrm{X}$ & $\mathrm{Y}$ & Z \\
\hline \multicolumn{6}{|c|}{ - - - - - - - - - - - - - - - - - - - - - - - - - - - - - - - - - - - - - - - - - - - - - - - - - - - - - - - - - - - } \\
\hline 1 & 15 & 0 & 1.438716 & -0.952587 & -0.139592 \\
\hline 2 & 6 & 0 & 0.414038 & -0.953029 & 1.152339 \\
\hline 3 & 14 & 0 & 1.092476 & -1.238351 & 2.854324 \\
\hline 4 & 1 & 0 & 2.447742 & -0.679781 & 3.147737 \\
\hline 5 & 1 & 0 & 0.154802 & -0.622939 & 3.837833 \\
\hline 6 & 1 & 0 & 1.164268 & -2.695840 & 3.161632 \\
\hline 7 & 7 & 0 & 0.985153 & -1.280692 & -1.719687 \\
\hline 8 & 7 & 0 & 2.671401 & 0.204341 & -0.359401 \\
\hline 9 & 6 & 0 & 3.909013 & -0.227829 & -1.013232 \\
\hline 10 & 1 & 0 & 4.346758 & 0.618697 & -1.556379 \\
\hline 11 & 1 & 0 & 3.694498 & -1.022711 & -1.730737 \\
\hline 12 & 1 & 0 & 4.652774 & -0.592967 & -0.288215 \\
\hline 13 & 6 & 0 & 2.861653 & 1.288071 & 0.604393 \\
\hline 14 & 1 & 0 & 3.472315 & 0.981604 & 1.466182 \\
\hline 15 & 1 & 0 & 1.901583 & 1.661324 & 0.961570 \\
\hline 16 & 1 & 0 & 3.376836 & 2.112928 & 0.096822 \\
\hline 17 & 6 & 0 & 0.755060 & -0.232661 & -2.718170 \\
\hline 18 & 1 & 0 & 1.055278 & -0.601963 & -3.706984 \\
\hline 19 & 1 & 0 & 1.359191 & 0.642603 & -2.474824 \\
\hline 20 & 1 & 0 & -0.303823 & 0.049873 & -2.737093 \\
\hline 21 & 6 & 0 & 0.306586 & -2.544690 & -2.002931 \\
\hline 22 & 1 & 0 & 0.524456 & -3.270959 & -1.215717 \\
\hline 23 & 1 & 0 & 0.669275 & -2.945029 & -2.959012 \\
\hline 24 & 1 & 0 & -0.778831 & -2.405137 & -2.062908 \\
\hline 25 & 6 & 0 & -1.461476 & -0.021148 & 1.329395 \\
\hline 26 & 6 & 0 & -1.052525 & 1.285596 & 1.521119 \\
\hline 27 & 6 & 0 & -0.822312 & 2.377006 & 0.576306 \\
\hline 28 & 8 & 0 & -0.147915 & 3.360629 & 0.855592 \\
\hline
\end{tabular}




$\begin{array}{rrrrrr}29 & 8 & 0 & -1.435875 & 2.233474 & -0.622949 \\ 30 & 6 & 0 & -1.233204 & 3.310937 & -1.543698 \\ 31 & 1 & 0 & -1.709247 & -0.556259 & 2.238993 \\ 32 & 6 & 0 & -2.221956 & -0.575701 & 0.161735 \\ 33 & 1 & 0 & -0.732397 & 1.550691 & 2.523908 \\ 34 & 1 & 0 & -0.169772 & 3.442003 & -1.764311 \\ 35 & 1 & 0 & -1.622075 & 4.248605 & -1.135894 \\ 36 & 1 & 0 & -1.779349 & 3.030116 & -2.445232 \\ 37 & 8 & 0 & -1.972277 & -0.496298 & -1.020988 \\ 38 & 8 & 0 & -3.303926 & -1.238817 & 0.640321 \\ 39 & 6 & 0 & -4.154621 & -1.827119 & -0.353532 \\ 40 & 1 & 0 & -4.985840 & -2.268684 & 0.197094 \\ 41 & 1 & 0 & -4.515162 & -1.067308 & -1.052003 \\ 42 & 1 & 0 & -3.616166 & -2.597201 & -0.913660 \\ ---------------------------------------------130\end{array}$

\section{cis-TScf-st-sc}

\begin{tabular}{|c|c|c|c|c|c|}
\hline Center & Atomic & Atomic & \multicolumn{3}{|c|}{ Coordinates (Angstroms) } \\
\hline Number & Number & Type & $\mathrm{X}$ & $\mathrm{Y}$ & Z \\
\hline & & & & & \\
\hline 1 & 15 & 0 & 1.661875 & -0.599187 & 0.112715 \\
\hline 2 & 6 & 0 & 0.775990 & -0.393681 & 1.464445 \\
\hline 3 & 14 & 0 & 1.431279 & 0.367436 & 3.009731 \\
\hline 4 & 1 & 0 & 2.354806 & 1.538820 & 2.873767 \\
\hline 5 & 1 & 0 & 0.276347 & 0.844972 & 3.826183 \\
\hline 6 & 1 & 0 & 2.163161 & -0.641479 & 3.828842 \\
\hline 7 & 7 & 0 & 1.428148 & -1.749947 & -1.072087 \\
\hline 8 & 7 & 0 & 2.456668 & 0.639890 & -0.739640 \\
\hline 9 & 6 & 0 & 3.718962 & 0.351668 & -1.421446 \\
\hline 10 & 1 & 0 & 3.797716 & 0.974209 & -2.321507 \\
\hline 11 & 1 & 0 & 3.746896 & -0.696710 & -1.723050 \\
\hline 12 & 1 & 0 & 4.588502 & 0.563557 & -0.779881 \\
\hline 13 & 6 & 0 & 2.317134 & 2.030103 & -0.316993 \\
\hline 14 & 1 & 0 & 3.080487 & 2.323028 & 0.419012 \\
\hline 15 & 1 & 0 & 1.331595 & 2.196499 & 0.119698 \\
\hline 16 & 1 & 0 & 2.423958 & 2.677773 & -1.196660 \\
\hline 17 & 6 & 0 & 0.751641 & -1.437038 & -2.335663 \\
\hline 18 & 1 & 0 & 1.208538 & -2.018541 & -3.146637 \\
\hline 19 & 1 & 0 & 0.855093 & -0.374716 & -2.558067 \\
\hline 20 & 1 & 0 & -0.314943 & -1.680270 & -2.267944 \\
\hline 21 & 6 & 0 & 1.375515 & -3.162478 & -0.686551 \\
\hline 22 & 1 & 0 & 1.907231 & -3.310694 & 0.256365 \\
\hline 23 & 1 & 0 & 1.867062 & -3.760856 & -1.465074 \\
\hline 24 & 1 & 0 & 0.342571 & -3.497978 & -0.560113 \\
\hline 25 & 6 & 0 & -1.413734 & -0.116464 & 1.471711 \\
\hline 26 & 6 & 0 & -1.469164 & 1.217223 & 1.151400 \\
\hline 27 & 6 & 0 & -1.531933 & 1.649230 & -0.234779 \\
\hline 28 & 8 & 0 & -1.547370 & 0.917403 & -1.218810 \\
\hline 29 & 8 & 0 & -1.548987 & 3.008859 & -0.338063 \\
\hline 30 & 6 & 0 & -1.636930 & 3.517875 & -1.672838 \\
\hline 31 & 1 & 0 & -1.459915 & -0.391498 & 2.520869 \\
\hline 32 & 6 & 0 & -1.988923 & -1.180751 & 0.561864 \\
\hline 33 & 1 & 0 & -1.349194 & 1.976193 & 1.916037 \\
\hline 34 & 1 & 0 & -2.549962 & 3.167967 & -2.163400 \\
\hline 35 & 1 & 0 & -0.776846 & 3.200222 & -2.270090 \\
\hline 36 & 1 & 0 & -1.650882 & 4.604328 & -1.573507 \\
\hline 37 & 8 & 0 & -1.440513 & -2.158707 & 0.107217 \\
\hline 38 & 8 & 0 & -3.311067 & -0.941956 & 0.411906 \\
\hline 39 & 6 & 0 & -4.007067 & -1.875414 & -0.427369 \\
\hline 40 & 1 & 0 & -5.051206 & -1.561054 & -0.408940 \\
\hline 41 & 1 & 0 & -3.612012 & -1.831435 & -1.446047 \\
\hline 42 & 1 & 0 & -3.904199 & -2.894680 & -0.045406 \\
\hline
\end{tabular}




\begin{tabular}{|c|c|c|c|c|c|}
\hline \multirow{2}{*}{$\begin{array}{l}\text { Center } \\
\text { Number }\end{array}$} & \multirow{3}{*}{$\begin{array}{l}\text { Atomic } \\
\text { Number }\end{array}$} & \multirow{2}{*}{$\begin{array}{l}\text { Atomic } \\
\text { Type }\end{array}$} & \multicolumn{3}{|c|}{ Coordinates (Angstroms) } \\
\hline & & & $\mathrm{X}$ & $\mathrm{Y}$ & $\mathrm{Z}$ \\
\hline & & & -------- & ------- & --------- \\
\hline 1 & 15 & 0 & -1.644236 & 0.646681 & 0.171224 \\
\hline 2 & 6 & 0 & -0.762586 & 0.060090 & 1.438868 \\
\hline 3 & 14 & 0 & -1.683162 & -0.811337 & 2.798354 \\
\hline 4 & 1 & 0 & -2.893386 & -1.596565 & 2.406416 \\
\hline 5 & 1 & 0 & -0.749197 & -1.769362 & 3.457515 \\
\hline 6 & 1 & 0 & -2.130051 & 0.159946 & 3.837193 \\
\hline 7 & 7 & 0 & -1.140038 & 1.905326 & -0.824945 \\
\hline 8 & 7 & 0 & -2.487198 & -0.343244 & -0.936011 \\
\hline 9 & 6 & 0 & -3.744739 & 0.172505 & -1.483539 \\
\hline 10 & 1 & 0 & -3.903844 & -0.249036 & -2.483609 \\
\hline 11 & 1 & 0 & -3.695293 & 1.259987 & -1.573033 \\
\hline 12 & 1 & 0 & -4.609451 & -0.094300 & -0.856462 \\
\hline 13 & 6 & 0 & -2.454541 & -1.800827 & -0.815765 \\
\hline 14 & 1 & 0 & -3.191029 & -2.180871 & -0.092777 \\
\hline 15 & 1 & 0 & -1.460385 & -2.143653 & -0.528681 \\
\hline 16 & 1 & 0 & -2.688201 & -2.230837 & -1.797593 \\
\hline 17 & 6 & 0 & -0.532120 & 1.685353 & -2.136739 \\
\hline 18 & 1 & 0 & -0.793100 & 2.515258 & -2.805537 \\
\hline 19 & 1 & 0 & -0.916500 & 0.758204 & -2.564580 \\
\hline 20 & 1 & 0 & 0.560843 & 1.614314 & -2.061584 \\
\hline 21 & 6 & 0 & -0.832319 & 3.191222 & -0.204901 \\
\hline 22 & 1 & 0 & -1.385462 & 3.294668 & 0.731896 \\
\hline 23 & 1 & 0 & -1.133893 & 4.002187 & -0.880682 \\
\hline 24 & 1 & 0 & 0.236857 & 3.295185 & 0.016577 \\
\hline 25 & 6 & 0 & 1.263971 & -0.561421 & 1.559625 \\
\hline 26 & 6 & 0 & 1.134691 & -1.747814 & 0.863500 \\
\hline 27 & 6 & 0 & 1.384314 & -2.063004 & -0.541116 \\
\hline 28 & 8 & 0 & 0.928054 & -3.056676 & -1.091037 \\
\hline 29 & 8 & 0 & 2.192829 & -1.183287 & -1.187393 \\
\hline 30 & 6 & 0 & 2.468911 & -1.509596 & -2.555046 \\
\hline 31 & 1 & 0 & 1.202507 & -0.657367 & 2.637719 \\
\hline 32 & 6 & 0 & 2.183747 & 0.579425 & 1.235895 \\
\hline 33 & 1 & 0 & 0.695401 & -2.585659 & 1.395445 \\
\hline 34 & 1 & 0 & 1.545976 & -1.553304 & -3.140386 \\
\hline 35 & 1 & 0 & 2.974335 & -2.476712 & -2.628069 \\
\hline 36 & 1 & 0 & 3.117356 & -0.712427 & -2.921586 \\
\hline 37 & 8 & 0 & 3.122364 & 0.829354 & 1.964534 \\
\hline 38 & 8 & 0 & 1.913746 & 1.280378 & 0.120545 \\
\hline 39 & 6 & 0 & 2.911556 & 2.261343 & -0.211748 \\
\hline 40 & 1 & 0 & 2.569046 & 2.727577 & -1.136063 \\
\hline 41 & 1 & 0 & 3.003689 & 3.005477 & 0.583509 \\
\hline 42 & 1 & 0 & 3.880992 & 1.779414 & -0.361635 \\
\hline
\end{tabular}

\section{trans-TScf-sc-sc}

\begin{tabular}{cccccc} 
Center & Atomic & Atomic & \multicolumn{2}{c}{ Coordinates } & (Angstroms) \\
Number & Number & Type & $\mathrm{X}$ & $\mathrm{Y}$ & $\mathrm{Z}$ \\
-1 & 15 & 0 & -2.173521 & -0.203770 & 0.343951 \\
1 & 6 & 0 & -0.636329 & -0.245120 & 0.875442 \\
3 & 14 & 0 & 0.008158 & 0.530310 & 2.434661 \\
4 & 1 & 0 & -0.889828 & 1.606247 & 2.971697 \\
5 & 1 & 0 & 1.316449 & 1.173424 & 2.169853 \\
6 & 1 & 0 & 0.131915 & -0.471901 & 3.524744 \\
7 & 6 & 0 & 0.944678 & -0.709602 & -0.613440 \\
8 & 6 & 0 & 1.269982 & 0.524598 & -1.112677 \\
9 & 6 & 0 & 1.907610 & -1.522695 & 0.224505
\end{tabular}




\begin{tabular}{|c|c|c|c|c|c|}
\hline 10 & 1 & 0 & 0.231693 & -1.311668 & -1.164826 \\
\hline 11 & 1 & 0 & 0.631758 & 0.993847 & -1.854007 \\
\hline 12 & 6 & 0 & 2.447868 & 1.271928 & -0.684220 \\
\hline 13 & 8 & 0 & 3.338159 & 0.867413 & 0.047456 \\
\hline 14 & 8 & 0 & 2.466730 & 2.515488 & -1.245704 \\
\hline 15 & 6 & 0 & 3.614920 & 3.306121 & -0.920199 \\
\hline 16 & 1 & 0 & 4.534383 & 2.808676 & -1.242866 \\
\hline 17 & 1 & 0 & 3.484617 & 4.249204 & -1.453527 \\
\hline 18 & 1 & 0 & 3.673436 & 3.481626 & 0.158008 \\
\hline 19 & 7 & 0 & -2.737451 & -1.302126 & -0.804719 \\
\hline 20 & 7 & 0 & -3.116205 & 1.178812 & -0.009547 \\
\hline 21 & 6 & 0 & -4.561912 & 1.114532 & 0.220546 \\
\hline 22 & 1 & 0 & -5.067512 & 1.815636 & -0.454757 \\
\hline 23 & 1 & 0 & -4.928748 & 0.107815 & 0.008345 \\
\hline 24 & 1 & 0 & -4.829429 & 1.379071 & 1.255051 \\
\hline 25 & 6 & 0 & -2.538431 & 2.499396 & 0.241374 \\
\hline 26 & 1 & 0 & -2.658351 & 2.822065 & 1.285539 \\
\hline 27 & 1 & 0 & -1.473429 & 2.491047 & 0.002081 \\
\hline 28 & 1 & 0 & -3.040338 & 3.229735 & -0.405538 \\
\hline 29 & 6 & 0 & -2.554369 & -2.729983 & -0.557631 \\
\hline 30 & 1 & 0 & -1.706301 & -3.137264 & -1.127273 \\
\hline 31 & 1 & 0 & -2.366768 & -2.906752 & 0.503442 \\
\hline 32 & 1 & 0 & -3.459539 & -3.276277 & -0.854901 \\
\hline 33 & 6 & 0 & -3.018956 & -0.951672 & -2.195148 \\
\hline 34 & 1 & 0 & -3.158944 & 0.126309 & -2.282727 \\
\hline 35 & 1 & 0 & -2.197220 & -1.260713 & -2.859622 \\
\hline 36 & 1 & 0 & -3.935038 & -1.454744 & -2.531648 \\
\hline 37 & 8 & 0 & 1.961516 & -1.594638 & 1.429593 \\
\hline 38 & 8 & 0 & 2.697278 & -2.241043 & -0.603876 \\
\hline 39 & 6 & 0 & 3.716558 & -3.013336 & 0.052380 \\
\hline 40 & 1 & 0 & 4.258792 & -3.519607 & -0.747158 \\
\hline 41 & 1 & 0 & 3.270935 & -3.740309 & 0.736870 \\
\hline 42 & 1 & 0 & 4.382485 & -2.352829 & 0.613234 \\
\hline
\end{tabular}

\section{trans-TScf-sc-st}

\begin{tabular}{|c|c|c|c|c|c|}
\hline Center & Atomic & Atomic & \multicolumn{3}{|c|}{ Coordinates (Angstroms) } \\
\hline Number & Number & Type & $\mathrm{X}$ & $\mathrm{Y}$ & Z \\
\hline-- & & & --------- & - & --------- \\
\hline 1 & 15 & 0 & 2.071542 & -0.041266 & 0.458069 \\
\hline 2 & 6 & 0 & 0.468304 & -0.093086 & 0.804249 \\
\hline 3 & 14 & 0 & -0.327046 & -1.063835 & 2.173194 \\
\hline 4 & 1 & 0 & 0.471894 & -2.246384 & 2.623775 \\
\hline 5 & 1 & 0 & -1.641175 & -1.576963 & 1.722455 \\
\hline 6 & 1 & 0 & -0.519610 & -0.206807 & 3.375524 \\
\hline 7 & 6 & 0 & -0.866740 & 0.559280 & -0.758082 \\
\hline 8 & 6 & 0 & -1.273315 & -0.643389 & -1.291039 \\
\hline 9 & 6 & 0 & -1.658498 & 1.522914 & 0.073389 \\
\hline 10 & 1 & 0 & -0.063374 & 1.055735 & -1.289049 \\
\hline 11 & 1 & 0 & -0.593409 & -1.117757 & -1.992565 \\
\hline 12 & 6 & 0 & -2.454662 & -1.480070 & -1.045866 \\
\hline 13 & 8 & 0 & -2.515899 & -2.645859 & -1.407330 \\
\hline 14 & 8 & 0 & -3.480185 & -0.839457 & -0.444440 \\
\hline 15 & 6 & 0 & -4.632052 & -1.645781 & -0.174704 \\
\hline 16 & 1 & 0 & -4.371297 & -2.489964 & 0.469848 \\
\hline 17 & 1 & 0 & -5.337362 & -0.984603 & 0.330005 \\
\hline 18 & 1 & 0 & -5.061914 & -2.031783 & -1.103836 \\
\hline 19 & 7 & 0 & 2.697828 & 1.234229 & -0.455320 \\
\hline 20 & 7 & 0 & 2.995314 & -1.364470 & -0.119043 \\
\hline 21 & 6 & 0 & 4.425096 & -1.388221 & 0.202739 \\
\hline 22 & 1 & 0 & 4.955219 & -1.976136 & -0.556319 \\
\hline 23 & 1 & 0 & 4.826286 & -0.372280 & 0.192716 \\
\hline 24 & 1 & 0 & 4.623031 & -1.838666 & 1.187692 \\
\hline
\end{tabular}




\begin{tabular}{|c|c|c|c|c|c|}
\hline 25 & 6 & 0 & 2.379090 & -2.691406 & -0.144184 \\
\hline 26 & 1 & 0 & 2.427414 & -3.199706 & 0.830050 \\
\hline 27 & 1 & 0 & 1.332411 & -2.613296 & -0.443326 \\
\hline 28 & 1 & 0 & 2.910244 & -3.308278 & -0.879440 \\
\hline 29 & 6 & 0 & 2.447249 & 2.593578 & 0.023552 \\
\hline 30 & 1 & 0 & 1.524836 & 3.018693 & -0.397074 \\
\hline 31 & 1 & 0 & 2.355363 & 2.594058 & 1.112399 \\
\hline 32 & 1 & 0 & 3.290928 & 3.237043 & -0.256595 \\
\hline 33 & 6 & 0 & 2.996463 & 1.134196 & -1.883653 \\
\hline 34 & 1 & 0 & 3.171006 & 0.091163 & -2.150344 \\
\hline 35 & 1 & 0 & 2.172390 & 1.527805 & -2.498785 \\
\hline 36 & 1 & 0 & 3.899310 & 1.713438 & -2.116373 \\
\hline 37 & 8 & 0 & -2.370796 & 1.294437 & 1.023016 \\
\hline 38 & 8 & 0 & -1.425176 & 2.781547 & -0.391078 \\
\hline 39 & 6 & 0 & -2.098225 & 3.824388 & 0.330297 \\
\hline 40 & 1 & 0 & -1.817861 & 4.753732 & -0.167128 \\
\hline 41 & 1 & 0 & -1.779330 & 3.834903 & 1.376310 \\
\hline 42 & 1 & 0 & -3.181292 & 3.680659 & 0.293317 \\
\hline
\end{tabular}

\section{trans-TScf-st-sc}

\begin{tabular}{|c|c|c|c|c|c|}
\hline \multirow{3}{*}{$\begin{array}{l}\text { Center } \\
\text { Number }\end{array}$} & \multirow{2}{*}{$\begin{array}{l}\text { Atomic } \\
\text { Number }\end{array}$} & \multirow{2}{*}{$\begin{array}{c}\text { Atomic } \\
\text { Type }\end{array}$} & \multicolumn{3}{|c|}{ Coordinates (Angstroms) } \\
\hline & & & $\mathrm{X}$ & $\mathrm{Y}$ & $\mathrm{Z}$ \\
\hline & & & -------- & --------- & -------- \\
\hline 1 & 15 & 0 & 2.116626 & -0.076258 & 0.390550 \\
\hline 2 & 6 & 0 & 0.519714 & -0.041124 & 0.749477 \\
\hline 3 & 14 & 0 & -0.311795 & -0.785052 & 2.227307 \\
\hline 4 & 1 & 0 & 0.322974 & -2.040327 & 2.740720 \\
\hline 5 & 1 & 0 & -1.713039 & -1.126635 & 1.886740 \\
\hline 6 & 1 & 0 & -0.306072 & 0.176738 & 3.365920 \\
\hline 7 & 6 & 0 & -0.759905 & 0.551704 & -0.854206 \\
\hline 8 & 6 & 0 & -1.485368 & -0.581840 & -1.154780 \\
\hline 9 & 6 & 0 & -1.290888 & 1.841425 & -0.303605 \\
\hline 10 & 1 & 0 & 0.096133 & 0.747619 & -1.489358 \\
\hline 11 & 1 & 0 & -0.997777 & -1.346005 & -1.752298 \\
\hline 12 & 6 & 0 & -2.866461 & -0.881262 & -0.774983 \\
\hline 13 & 8 & 0 & -3.704077 & -0.120305 & -0.323457 \\
\hline 14 & 8 & 0 & -3.148058 & -2.189732 & -1.063434 \\
\hline 15 & 6 & 0 & -4.495612 & -2.587440 & -0.795365 \\
\hline 16 & 1 & 0 & -5.203083 & -1.984092 & -1.372291 \\
\hline 17 & 1 & 0 & -4.558857 & -3.635912 & -1.091593 \\
\hline 18 & 1 & 0 & -4.730443 & -2.476983 & 0.267578 \\
\hline 19 & 7 & 0 & 2.763537 & 1.066249 & -0.661461 \\
\hline 20 & 7 & 0 & 3.033243 & -1.471590 & 0.008456 \\
\hline 21 & 6 & 0 & 4.464136 & -1.464560 & 0.327824 \\
\hline 22 & 1 & 0 & 4.987362 & -2.153221 & -0.346498 \\
\hline 23 & 1 & 0 & 4.874435 & -0.462820 & 0.182439 \\
\hline 24 & 1 & 0 & 4.659715 & -1.781108 & 1.363895 \\
\hline 25 & 6 & 0 & 2.401776 & -2.780040 & 0.176540 \\
\hline 26 & 1 & 0 & 2.440695 & -3.140066 & 1.215299 \\
\hline 27 & 1 & 0 & 1.356175 & -2.730988 & -0.132786 \\
\hline 28 & 1 & 0 & 2.925769 & -3.505206 & -0.458091 \\
\hline 29 & 6 & 0 & 2.535056 & 2.483332 & -0.366250 \\
\hline 30 & 1 & 0 & 1.659407 & 2.878001 & -0.898124 \\
\hline 31 & 1 & 0 & 2.370846 & 2.618400 & 0.705320 \\
\hline 32 & 1 & 0 & 3.420957 & 3.060308 & -0.660463 \\
\hline 33 & 6 & 0 & 3.156480 & 0.782537 & -2.041737 \\
\hline 34 & 1 & 0 & 3.275476 & -0.292821 & -2.178594 \\
\hline 35 & 1 & 0 & 2.403250 & 1.153695 & -2.752489 \\
\hline 36 & 1 & 0 & 4.110335 & 1.275867 & -2.269777 \\
\hline 37 & 8 & 0 & -1.108647 & 2.898073 & -0.880722 \\
\hline 38 & 8 & 0 & -1.933493 & 1.729524 & 0.864667 \\
\hline 39 & 6 & 0 & -2.534819 & 2.933322 & 1.361730 \\
\hline
\end{tabular}




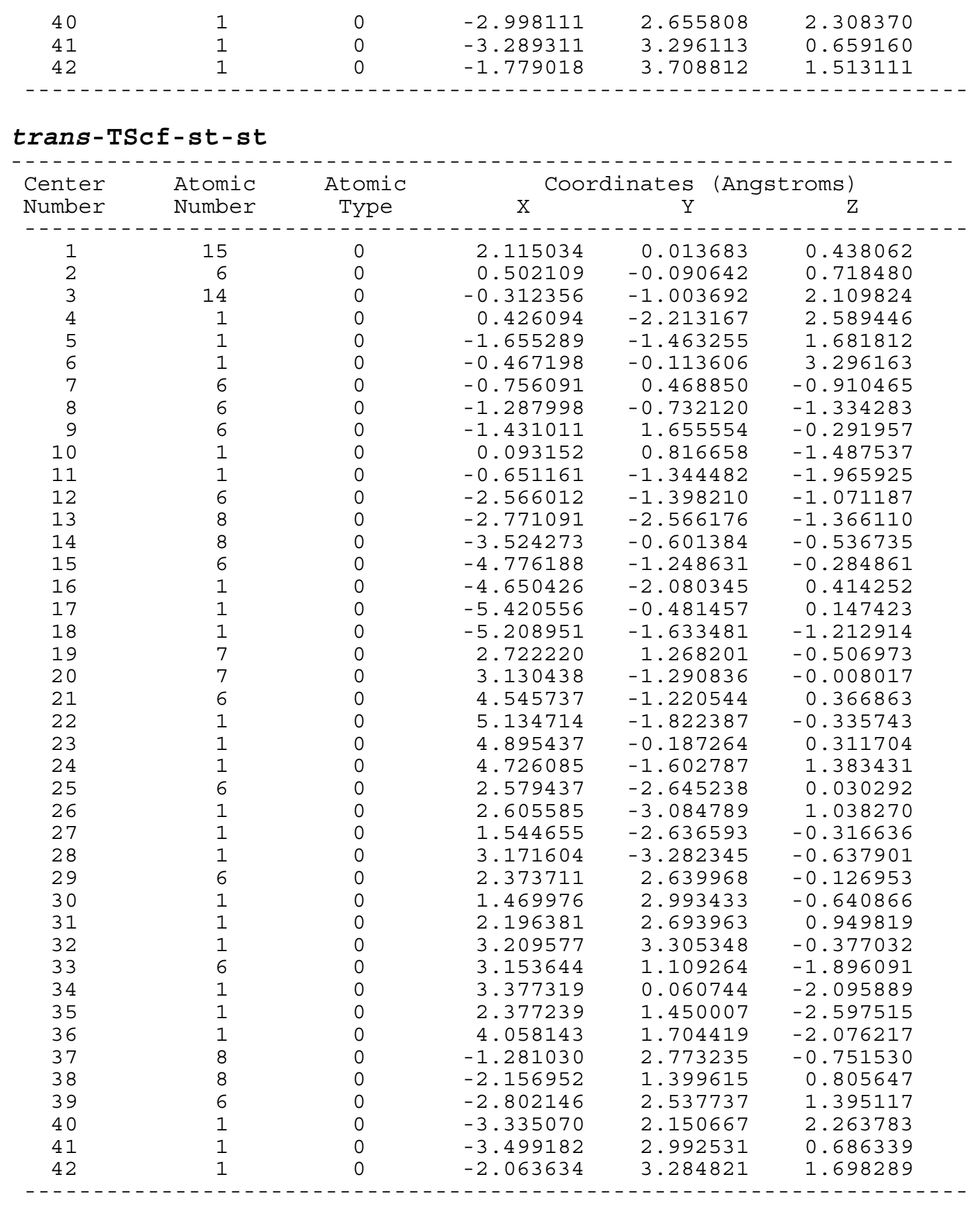

\section{cis-TSdb}

\begin{tabular}{|c|c|c|c|c|c|}
\hline \multirow{2}{*}{$\begin{array}{l}\text { Center } \\
\text { Number }\end{array}$} & \multirow{2}{*}{$\begin{array}{l}\text { Atomic } \\
\text { Number }\end{array}$} & \multirow{2}{*}{$\begin{array}{l}\text { Atomic } \\
\text { Type }\end{array}$} & \multicolumn{3}{|c|}{ Coordinates (Angstroms) } \\
\hline & & & $\mathrm{X}$ & $Y$ & $\mathrm{Z}$ \\
\hline------ & 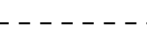 & $1+$ & -------- & ---------- & -------- \\
\hline 1 & 6 & 0 & -0.561698 & -0.845598 & -1.999123 \\
\hline 2 & 6 & 0 & -1.462710 & -1.586868 & -1.273939 \\
\hline 3 & 6 & 0 & 0.970152 & -0.282598 & -0.557148 \\
\hline 4 & 6 & 0 & -2.483026 & -0.929721 & -0.481662 \\
\hline 5 & 8 & 0 & -2.606608 & 0.280719 & -0.306599 \\
\hline
\end{tabular}




\begin{tabular}{|c|c|c|c|c|c|}
\hline 6 & 15 & 0 & 0.792813 & 1.231691 & -0.011186 \\
\hline 7 & 14 & 0 & 1.906824 & -1.748264 & 0.098624 \\
\hline 8 & 7 & 0 & 0.374670 & 2.507130 & -1.024891 \\
\hline 9 & 8 & 0 & -3.334650 & -1.831047 & 0.094588 \\
\hline 10 & 6 & 0 & -4.385268 & -1.263458 & 0.878940 \\
\hline 11 & 7 & 0 & 0.237689 & 1.793208 & 1.497122 \\
\hline 12 & 6 & 0 & -0.927487 & 3.175707 & -0.971254 \\
\hline 13 & 6 & 0 & 1.099231 & 2.677528 & -2.279797 \\
\hline 14 & 6 & 0 & 0.902989 & 2.943145 & 2.108965 \\
\hline 15 & 6 & 0 & -0.329440 & 0.841399 & 2.445767 \\
\hline 16 & 6 & 0 & 1.031235 & -2.635595 & 1.531312 \\
\hline 17 & 6 & 0 & 3.629219 & -1.199240 & 0.681981 \\
\hline 18 & 6 & 0 & 2.143738 & -3.012715 & -1.297385 \\
\hline 19 & 1 & 0 & -0.791475 & 0.189109 & -2.221195 \\
\hline 20 & 1 & 0 & 0.120304 & -1.334862 & -2.684044 \\
\hline 21 & 1 & 0 & -1.392469 & -2.667633 & -1.209953 \\
\hline 22 & 1 & 0 & -4.975833 & -2.108243 & 1.237931 \\
\hline 23 & 1 & 0 & -5.006745 & -0.593552 & 0.276898 \\
\hline 24 & 1 & 0 & -3.983863 & -0.696112 & 1.724783 \\
\hline 25 & 1 & 0 & -0.791996 & 4.262118 & -1.058430 \\
\hline 26 & 1 & 0 & -1.421153 & 2.947039 & -0.027966 \\
\hline 27 & 1 & 0 & -1.577367 & 2.835446 & -1.788832 \\
\hline 28 & 1 & 0 & 1.259945 & 3.747415 & -2.469552 \\
\hline 29 & 1 & 0 & 0.546066 & 2.258383 & -3.133009 \\
\hline 30 & 1 & 0 & 2.069586 & 2.179319 & -2.224699 \\
\hline 31 & 1 & 0 & 1.760958 & 2.646658 & 2.732772 \\
\hline 32 & 1 & 0 & 0.187784 & 3.481499 & 2.743186 \\
\hline 33 & 1 & 0 & 1.253001 & 3.625946 & 1.331476 \\
\hline 34 & 1 & 0 & 0.436314 & 0.323039 & 3.043287 \\
\hline 35 & 1 & 0 & -0.927363 & 0.103219 & 1.910308 \\
\hline 36 & 1 & 0 & -0.985709 & 1.387506 & 3.135129 \\
\hline 37 & 1 & 0 & 1.571201 & -3.548769 & 1.814108 \\
\hline 38 & 1 & 0 & 0.010595 & -2.922720 & 1.252947 \\
\hline 39 & 1 & 0 & 0.964550 & -2.000557 & 2.422376 \\
\hline 40 & 1 & 0 & 4.233995 & -2.065300 & 0.980331 \\
\hline 41 & 1 & 0 & 3.566701 & -0.523500 & 1.543211 \\
\hline 42 & 1 & 0 & 4.168339 & -0.673098 & -0.114090 \\
\hline 43 & 1 & 0 & 2.756082 & -3.855436 & -0.952079 \\
\hline 44 & 1 & 0 & 2.652195 & -2.563724 & -2.158884 \\
\hline 45 & 1 & 0 & 1.190556 & -3.424510 & -1.648408 \\
\hline
\end{tabular}

\section{trans-TSdb}

\begin{tabular}{cccccc}
$\begin{array}{c}\text { Center } \\
\text { Number }\end{array}$ & $\begin{array}{c}\text { Atomic } \\
\text { Number }\end{array}$ & $\begin{array}{c}\text { Atomic } \\
\text { Type }\end{array}$ & \multicolumn{2}{c}{ Coordinates } & (Angstroms) \\
- & 6 & 0 & -0.692089 & -0.634404 & -1.893863 \\
1 & 6 & 0 & -1.302091 & -1.520753 & -1.047331 \\
2 & 6 & 0 & -2.639186 & -1.281229 & -0.516601 \\
3 & 8 & 0 & -3.356433 & -0.315186 & -0.739801 \\
4 & 8 & 0 & -3.033843 & -2.307848 & 0.293141 \\
5 & 6 & 0 & -4.349349 & -2.178777 & 0.841287 \\
6 & 6 & 0 & 0.382735 & 0.707860 & -0.498981 \\
7 & 15 & 0 & 1.792947 & 0.095212 & 0.046806 \\
8 & 14 & 0 & -0.614472 & 2.195936 & 0.045619 \\
9 & 7 & 0 & 2.809042 & -0.820364 & -0.959253 \\
10 & 7 & 0 & 1.990679 & -0.758123 & 1.526383 \\
11 & 6 & 0 & 3.281429 & -0.196072 & -2.190811 \\
12 & 6 & 0 & 2.782239 & -2.281437 & -0.996619 \\
13 & 6 & 0 & 0.885860 & -0.762978 & 2.480665 \\
14 & 6 & 0 & 3.309837 & -0.735948 & 2.161766 \\
15 & 6 & 0 & -1.746472 & 2.758444 & -1.361809 \\
16 & 6 & 0 & 0.613482 & 3.595342 & 0.431514 \\
17 & & & & &
\end{tabular}




\begin{tabular}{|c|c|c|c|c|c|}
\hline 18 & 6 & 0 & -1.702639 & 1.906718 & 1.573366 \\
\hline 19 & 1 & 0 & -1.265055 & 0.175521 & -2.328997 \\
\hline 20 & 1 & 0 & 0.211024 & -0.914513 & -2.421190 \\
\hline 21 & 1 & 0 & -0.797336 & -2.422091 & -0.713749 \\
\hline 22 & 1 & 0 & -4.513518 & -3.082779 & 1.430132 \\
\hline 23 & 1 & 0 & -5.097365 & -2.099144 & 0.047083 \\
\hline 24 & 1 & 0 & -4.422319 & -1.291711 & 1.478029 \\
\hline 25 & 1 & 0 & 4.294172 & -0.553991 & -2.418957 \\
\hline 26 & 1 & 0 & 3.312681 & 0.889718 & -2.073313 \\
\hline 27 & 1 & 0 & 2.635522 & -0.427177 & -3.051646 \\
\hline 28 & 1 & 0 & 3.792268 & -2.668009 & -1.186597 \\
\hline 29 & 1 & 0 & 2.119861 & -2.657668 & -1.792930 \\
\hline 30 & 1 & 0 & 2.435255 & -2.666483 & -0.036904 \\
\hline 31 & 1 & 0 & 0.869474 & 0.136537 & 3.116596 \\
\hline 32 & 1 & 0 & 0.990230 & -1.637622 & 3.135193 \\
\hline 33 & 1 & 0 & -0.065251 & -0.830664 & 1.950654 \\
\hline 34 & 1 & 0 & 3.447545 & 0.145748 & 2.807900 \\
\hline 35 & 1 & 0 & 4.090205 & -0.738041 & 1.397773 \\
\hline 36 & 1 & 0 & 3.429521 & -1.633722 & 2.781004 \\
\hline 37 & 1 & 0 & -2.232327 & 3.708027 & -1.102654 \\
\hline 38 & 1 & 0 & -2.534685 & 2.020004 & -1.542800 \\
\hline 39 & 1 & 0 & -1.189518 & 2.913338 & -2.293724 \\
\hline 40 & 1 & 0 & 0.076078 & 4.523385 & 0.665942 \\
\hline 41 & 1 & 0 & 1.270598 & 3.794753 & -0.422345 \\
\hline 42 & 1 & 0 & 1.248507 & 3.347190 & 1.289972 \\
\hline 43 & 1 & 0 & -2.258768 & 2.822201 & 1.814104 \\
\hline 44 & 1 & 0 & -1.118626 & 1.630275 & 2.458463 \\
\hline 45 & 1 & 0 & -2.438125 & 1.118709 & 1.380080 \\
\hline
\end{tabular}

\section{cis-TS' db}

\begin{tabular}{|c|c|c|c|c|c|}
\hline \multirow{2}{*}{$\begin{array}{l}\text { Center } \\
\text { Number }\end{array}$} & \multirow{2}{*}{$\begin{array}{l}\text { Atomic } \\
\text { Number }\end{array}$} & \multirow{2}{*}{$\begin{array}{c}\text { Atomic } \\
\text { Type }\end{array}$} & \multicolumn{3}{|c|}{ Coordinates (Angstroms) } \\
\hline & & & $\mathrm{X}$ & $\mathrm{Y}$ & Z \\
\hline------ & & & -------- & --------1 & -------- \\
\hline 1 & 6 & 0 & -0.932136 & -0.267283 & -0.555058 \\
\hline 2 & 15 & 0 & 0.155014 & -1.374579 & -0.059992 \\
\hline 3 & 7 & 0 & 0.966229 & -1.428241 & 1.450153 \\
\hline 4 & 14 & 0 & -2.660333 & 0.096721 & 0.039132 \\
\hline 5 & 7 & 0 & 1.361051 & -1.966869 & -1.088610 \\
\hline 6 & 6 & 0 & -0.168385 & 1.354571 & -1.805653 \\
\hline 7 & 6 & 0 & -0.041906 & 2.364757 & -0.887529 \\
\hline 8 & 6 & 0 & 1.141936 & 2.611618 & -0.075487 \\
\hline 9 & 8 & 0 & 1.224508 & 3.456607 & 0.802658 \\
\hline 10 & 1 & 0 & 0.705779 & 0.812493 & -2.143630 \\
\hline 11 & 1 & 0 & -1.021660 & 1.331642 & -2.472250 \\
\hline 12 & 1 & 0 & -0.877822 & 3.015734 & -0.652690 \\
\hline 13 & 8 & 0 & 2.197883 & 1.792977 & -0.391605 \\
\hline 14 & 6 & 0 & -3.592717 & 1.084599 & -1.287745 \\
\hline 15 & 6 & 0 & -3.580470 & -1.545041 & 0.291709 \\
\hline 16 & 6 & 0 & -2.749321 & 1.095753 & 1.651791 \\
\hline 17 & 6 & 0 & 0.614717 & -0.446211 & 2.471492 \\
\hline 18 & 6 & 0 & 1.315145 & -2.742312 & 1.994090 \\
\hline 19 & 6 & 0 & 2.757558 & -1.538913 & -1.015220 \\
\hline 20 & 6 & 0 & 0.950343 & -2.458735 & -2.400150 \\
\hline 21 & 6 & 0 & 3.390673 & 2.044647 & 0.357306 \\
\hline 22 & 1 & 0 & 4.133826 & 1.342023 & -0.023840 \\
\hline 23 & 1 & 0 & 3.731270 & 3.073941 & 0.212601 \\
\hline 24 & 1 & 0 & 3.225794 & 1.881685 & 1.426639 \\
\hline 25 & 1 & 0 & -4.641477 & 1.213592 & -0.991443 \\
\hline 26 & 1 & 0 & -3.174685 & 2.086471 & -1.438895 \\
\hline 27 & 1 & 0 & -3.583153 & 0.566863 & -2.254273 \\
\hline 28 & 1 & 0 & -3.784941 & 1.389651 & 1.867506 \\
\hline 29 & 1 & 0 & -2.385051 & 0.518786 & 2.509757 \\
\hline
\end{tabular}




\begin{tabular}{|c|c|c|c|c|c|}
\hline 30 & 1 & 0 & -2.148691 & 2.010634 & 1.590010 \\
\hline 31 & 1 & 0 & -4.630066 & -1.364826 & 0.557405 \\
\hline 32 & 1 & 0 & -3.563706 & -2.154600 & -0.618742 \\
\hline 33 & 1 & 0 & -3.127257 & -2.136283 & 1.095847 \\
\hline 34 & 1 & 0 & 1.458897 & -0.344063 & 3.165170 \\
\hline 35 & 1 & 0 & 0.419517 & 0.523995 & 2.012659 \\
\hline 36 & 1 & 0 & -0.270100 & -0.740412 & 3.057622 \\
\hline 37 & 1 & 0 & 2.181634 & -2.640883 & 2.659404 \\
\hline 38 & 1 & 0 & 0.489859 & -3.187871 & 2.572159 \\
\hline 39 & 1 & 0 & 1.582523 & -3.421914 & 1.181993 \\
\hline 40 & 1 & 0 & 2.963070 & -0.707302 & -1.705292 \\
\hline 41 & 1 & 0 & 2.987159 & -1.214583 & -0.000241 \\
\hline 42 & 1 & 0 & 3.413530 & -2.379326 & -1.277284 \\
\hline 43 & 1 & 0 & 1.021941 & -1.681795 & -3.176003 \\
\hline 44 & 1 & 0 & 1.594917 & -3.297420 & -2.694618 \\
\hline 45 & 1 & 0 & -0.084367 & -2.807242 & -2.364030 \\
\hline
\end{tabular}

\section{trans-TS' db}

\begin{tabular}{|c|c|c|c|c|c|}
\hline \multirow{2}{*}{$\begin{array}{l}\text { Center } \\
\text { Number }\end{array}$} & \multirow{2}{*}{$\begin{array}{l}\text { Atomic } \\
\text { Number }\end{array}$} & \multirow{2}{*}{$\begin{array}{c}\text { Atomic } \\
\text { Type }\end{array}$} & \multicolumn{3}{|c|}{ Coordinates (Angstroms) } \\
\hline & & & $\mathrm{X}$ & $\mathrm{Y}$ & Z \\
\hline & & & --------- & & \\
\hline 1 & 6 & 0 & -0.270185 & 0.598350 & -0.541943 \\
\hline 2 & 14 & 0 & 0.907175 & 1.978212 & -0.095665 \\
\hline 3 & 6 & 0 & 1.840580 & 1.724296 & 1.538769 \\
\hline 4 & 15 & 0 & -1.795451 & 0.284165 & -0.040408 \\
\hline 5 & 7 & 0 & -2.868359 & -0.601112 & -1.015456 \\
\hline 6 & 6 & 0 & -3.185966 & -0.062126 & -2.333644 \\
\hline 7 & 7 & 0 & -2.187513 & -0.369686 & 1.502003 \\
\hline 8 & 6 & 0 & -3.522609 & -0.097639 & 2.038674 \\
\hline 9 & 6 & 0 & -1.148571 & -0.415605 & 2.527125 \\
\hline 10 & 6 & 0 & 2.191827 & 2.196098 & -1.472024 \\
\hline 11 & 6 & 0 & -0.091785 & 3.589570 & 0.032502 \\
\hline 12 & 6 & 0 & -3.048963 & -2.046487 & -0.895087 \\
\hline 13 & 6 & 0 & 0.664693 & -1.075453 & -1.639115 \\
\hline 14 & 6 & 0 & 1.027053 & -1.907958 & -0.613763 \\
\hline 15 & 1 & 0 & 0.318866 & -2.620022 & -0.200925 \\
\hline 16 & 1 & 0 & -0.238433 & -1.272789 & -2.203081 \\
\hline 17 & 1 & 0 & 1.402667 & -0.458421 & -2.136619 \\
\hline 18 & 6 & 0 & 2.319755 & -1.923687 & 0.065287 \\
\hline 19 & 1 & 0 & 2.819877 & 3.074052 & -1.272830 \\
\hline 20 & 1 & 0 & 2.852106 & 1.325782 & -1.546929 \\
\hline 21 & 1 & 0 & 1.710534 & 2.345759 & -2.445823 \\
\hline 22 & 1 & 0 & 2.582086 & 2.521059 & 1.682459 \\
\hline 23 & 1 & 0 & 1.165281 & 1.740823 & 2.401794 \\
\hline 24 & 1 & 0 & 2.375267 & 0.768532 & 1.550997 \\
\hline 25 & 1 & 0 & 0.577463 & 4.443063 & 0.201874 \\
\hline 26 & 1 & 0 & -0.656162 & 3.782243 & -0.886618 \\
\hline 27 & 1 & 0 & -0.807735 & 3.552430 & 0.861552 \\
\hline 28 & 1 & 0 & -1.420319 & -1.178481 & 3.267243 \\
\hline 29 & 1 & 0 & -0.191335 & -0.687139 & 2.080399 \\
\hline 30 & 1 & 0 & -1.031310 & 0.543619 & 3.056428 \\
\hline 31 & 1 & 0 & -3.798882 & -0.892977 & 2.741998 \\
\hline 32 & 1 & 0 & -3.570356 & 0.864053 & 2.574234 \\
\hline 33 & 1 & 0 & -4.254019 & -0.085702 & 1.227740 \\
\hline 34 & 1 & 0 & -2.406340 & -2.599562 & -1.599052 \\
\hline 35 & 1 & 0 & -2.812717 & -2.362099 & 0.121930 \\
\hline 36 & 1 & 0 & -4.092443 & -2.311131 & -1.111690 \\
\hline 37 & 1 & 0 & -2.529561 & -0.464537 & -3.120594 \\
\hline 38 & 1 & 0 & -4.222795 & -0.313819 & -2.593563 \\
\hline 39 & 1 & 0 & -3.081995 & 1.025611 & -2.329471 \\
\hline 40 & 8 & 0 & 2.590884 & -2.644078 & 1.013227 \\
\hline 41 & 8 & 0 & 3.230971 & -1.059787 & -0.476016 \\
\hline
\end{tabular}




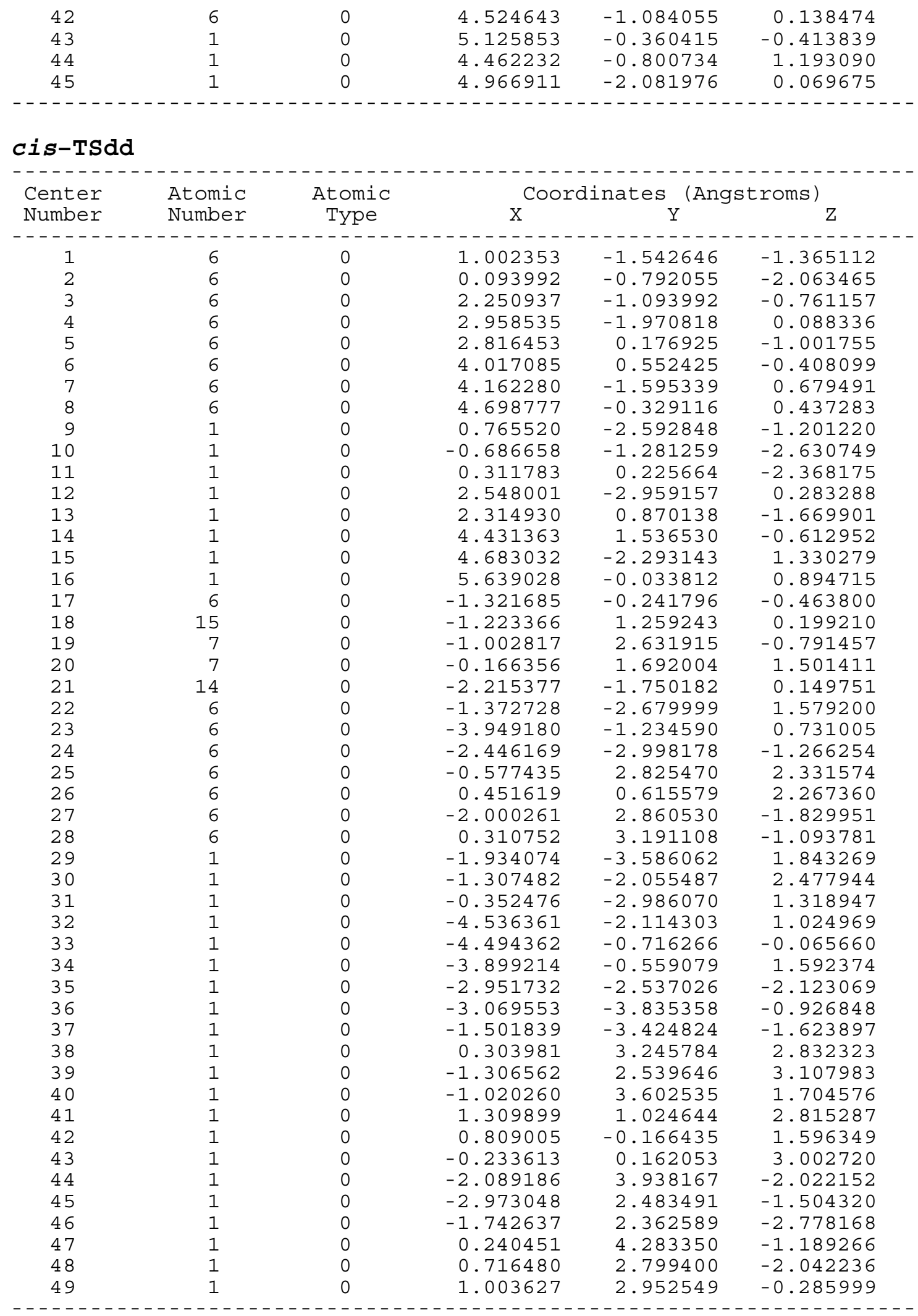

trans-TSdd 


\begin{tabular}{|c|c|c|c|c|c|}
\hline \multirow{2}{*}{$\begin{array}{l}\text { Center } \\
\text { Number }\end{array}$} & \multirow{2}{*}{$\begin{array}{l}\text { Atomic } \\
\text { Number }\end{array}$} & \multirow{2}{*}{$\begin{array}{c}\text { Atomic } \\
\text { Type }\end{array}$} & \multicolumn{3}{|c|}{ Coordinates (Angstroms) } \\
\hline & & & $\mathrm{X}$ & $\mathrm{Y}$ & $\mathrm{Z}$ \\
\hline & & & -------- & $-6-2-6$ & ----------1 \\
\hline 1 & 6 & 0 & -0.993195 & -1.144938 & -1.273741 \\
\hline 2 & 6 & 0 & -0.301794 & -0.197216 & -1.978425 \\
\hline 3 & 6 & 0 & -2.358068 & -1.072204 & -0.763341 \\
\hline 4 & 6 & 0 & -3.260650 & -0.042534 & -1.105599 \\
\hline 5 & 6 & 0 & -4.549867 & -0.016355 & -0.584204 \\
\hline 6 & 6 & 0 & -4.985147 & -1.023267 & 0.283939 \\
\hline 7 & 6 & 0 & -4.115488 & -2.063449 & 0.616868 \\
\hline 8 & 6 & 0 & -2.822673 & -2.087517 & 0.098784 \\
\hline 9 & 1 & 0 & -0.465529 & -2.066516 & -1.029983 \\
\hline 10 & 1 & 0 & -0.776524 & 0.705324 & -2.346499 \\
\hline 11 & 1 & 0 & 0.626361 & -0.460703 & -2.467144 \\
\hline 12 & 1 & 0 & -2.948092 & 0.739685 & -1.790285 \\
\hline 13 & 1 & 0 & -5.222331 & 0.792657 & -0.857494 \\
\hline 14 & 1 & 0 & -5.992872 & -0.998677 & 0.689433 \\
\hline 15 & 1 & 0 & -4.444618 & -2.856899 & 1.282936 \\
\hline 16 & 1 & 0 & -2.151381 & -2.901160 & 0.364364 \\
\hline 17 & 6 & 0 & 0.826727 & 0.796567 & -0.318077 \\
\hline 18 & 15 & 0 & 2.156536 & -0.012967 & 0.217182 \\
\hline 19 & 7 & 0 & 3.274478 & -0.681578 & -0.895852 \\
\hline 20 & 7 & 0 & 1.978720 & -1.394750 & 1.259947 \\
\hline 21 & 14 & 0 & -0.094899 & 2.246208 & 0.386683 \\
\hline 22 & 6 & 0 & -1.433824 & 1.834604 & 1.670458 \\
\hline 23 & 6 & 0 & 1.170543 & 3.388537 & 1.227664 \\
\hline 24 & 6 & 0 & -0.912975 & 3.250279 & -1.005485 \\
\hline 25 & 6 & 0 & 3.171890 & -1.840939 & 1.981543 \\
\hline 26 & 6 & 0 & 0.774622 & -1.466046 & 2.080767 \\
\hline 27 & 6 & 0 & 4.034485 & 0.266676 & -1.701569 \\
\hline 28 & 6 & 0 & 3.086660 & -1.982383 & -1.530856 \\
\hline 29 & 1 & 0 & -1.948847 & 2.749797 & 1.991573 \\
\hline 30 & 1 & 0 & -0.994935 & 1.371339 & 2.562107 \\
\hline 31 & 1 & 0 & -2.192671 & 1.150220 & 1.277682 \\
\hline 32 & 1 & 0 & 0.680086 & 4.303601 & 1.584662 \\
\hline 33 & 1 & 0 & 1.969720 & 3.679082 & 0.537340 \\
\hline 34 & 1 & 0 & 1.635925 & 2.895310 & 2.088231 \\
\hline 35 & 1 & 0 & -0.182649 & 3.507741 & -1.781767 \\
\hline 36 & 1 & 0 & -1.321675 & 4.188158 & -0.607825 \\
\hline 37 & 1 & 0 & -1.741447 & 2.718922 & -1.487835 \\
\hline 38 & 1 & 0 & 3.052004 & -2.894847 & 2.264019 \\
\hline 39 & 1 & 0 & 3. 348209 & -1.260983 & 2.902942 \\
\hline 40 & 1 & 0 & 4.049280 & -1.752867 & 1.338152 \\
\hline 41 & 1 & 0 & 0.623603 & -2.506445 & 2.397893 \\
\hline 42 & 1 & 0 & -0.093004 & -1.142278 & 1.505122 \\
\hline 43 & 1 & 0 & 0.838992 & -0.844529 & 2.990170 \\
\hline 44 & 1 & 0 & 5.014786 & -0.162828 & -1.949258 \\
\hline 45 & 1 & 0 & 4.194662 & 1.190369 & -1.138927 \\
\hline 46 & 1 & 0 & 3.526277 & 0.526167 & -2.644117 \\
\hline 47 & 1 & 0 & 4.062410 & -2.453951 & -1.713285 \\
\hline 48 & 1 & 0 & 2.569550 & -1.902483 & -2.501945 \\
\hline 49 & 1 & 0 & 2.505395 & -2.630204 & -0.872564 \\
\hline
\end{tabular}

\section{cis-TSec}

\begin{tabular}{|c|c|c|c|c|c|}
\hline \multirow{2}{*}{$\begin{array}{l}\text { Center } \\
\text { Number }\end{array}$} & \multirow{2}{*}{$\begin{array}{l}\text { Atomic } \\
\text { Number }\end{array}$} & \multirow{2}{*}{$\begin{array}{c}\text { Atomic } \\
\text { Type }\end{array}$} & \multicolumn{3}{|c|}{ Coordinates (Angstroms) } \\
\hline & & & $\mathrm{X}$ & $\mathrm{Y}$ & $\mathrm{Z}$ \\
\hline 年 & - & & -------- & -------- & --------- \\
\hline 1 & 6 & 0 & -2.628634 & -0.030853 & -1.566053 \\
\hline 2 & 6 & 0 & -1.485345 & 0.510691 & -2.104600 \\
\hline 3 & 6 & 0 & -2.614031 & -1.386099 & -1.018135 \\
\hline 4 & 7 & 0 & -3.818857 & -1.940756 & -0.616166 \\
\hline
\end{tabular}




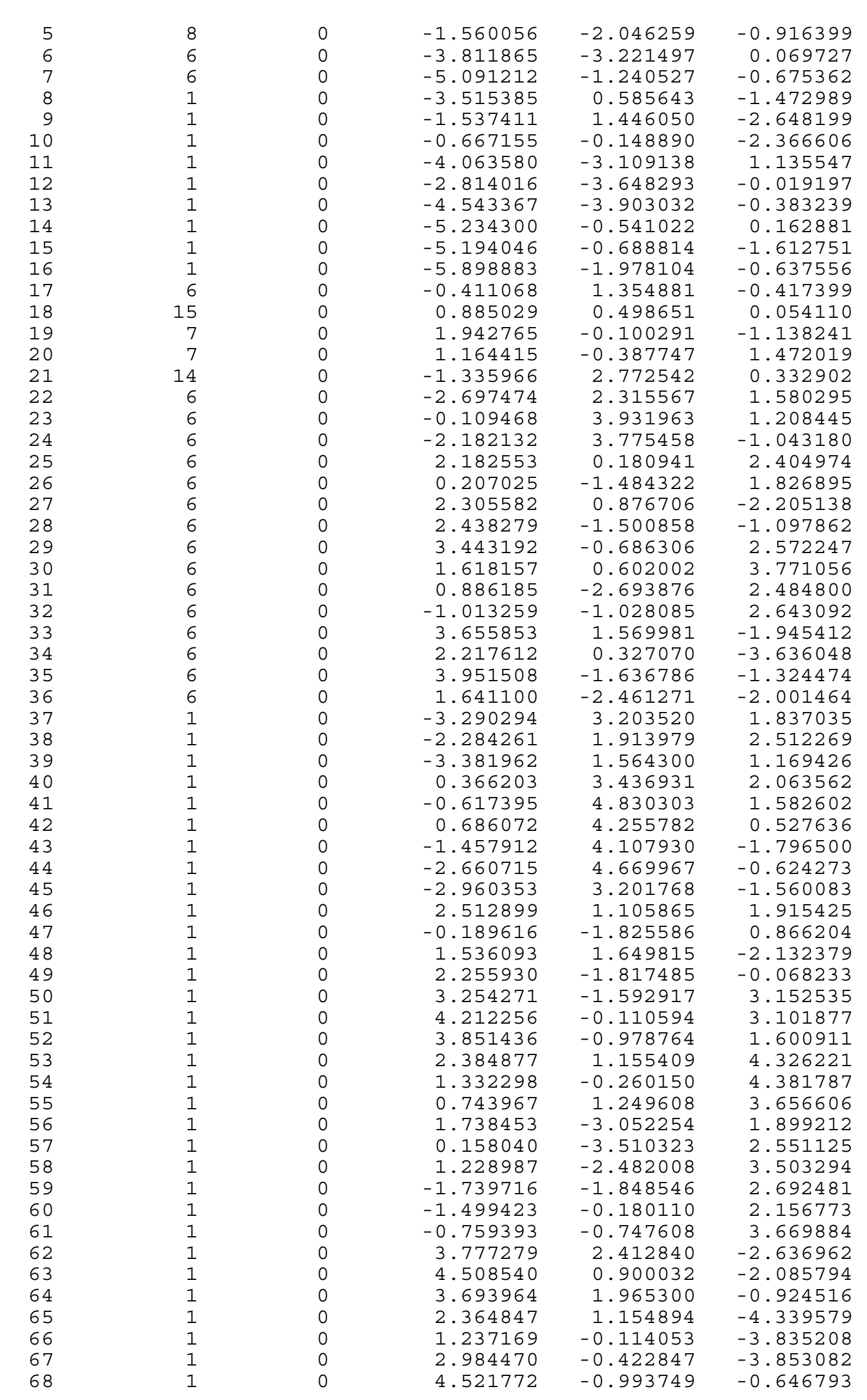




$\begin{array}{llllll}69 & 1 & 0 & 4.247921 & -1.401376 & -2.351236 \\ 70 & 1 & 0 & 4.246059 & -2.675421 & -1.134131 \\ 71 & 1 & 0 & 1.848235 & -2.299195 & -3.063435 \\ 72 & 1 & 0 & 0.566206 & -2.356786 & -1.828335 \\ 73 & 1 & 0 & 1.925869 & -3.495276 & -1.767027 \\ -\end{array}$

\section{trans-TSec}

\begin{tabular}{|c|c|c|c|c|c|}
\hline \multirow{2}{*}{$\begin{array}{l}\text { Center } \\
\text { Number }\end{array}$} & \multirow{2}{*}{$\begin{array}{l}\text { Atomic } \\
\text { Number }\end{array}$} & \multirow{2}{*}{$\begin{array}{c}\text { Atomic } \\
\text { Type }\end{array}$} & \multicolumn{3}{|c|}{ Coordinates (Angstroms) } \\
\hline & & & $\mathrm{X}$ & $\mathrm{Y}$ & $\mathrm{Z}$ \\
\hline & & & -------- & -------1 & ------- \\
\hline 1 & 6 & 0 & 2.202924 & -1.281892 & 2.377234 \\
\hline 2 & 14 & 0 & 0.917967 & -1.909036 & 1.129081 \\
\hline 3 & 6 & 0 & 1.390467 & -3.651003 & 0.546081 \\
\hline 4 & 6 & 0 & 0.679721 & -0.732160 & -0.406608 \\
\hline 5 & 15 & 0 & -0.775725 & 0.443370 & -0.782364 \\
\hline 6 & 7 & 0 & -0.745405 & 1.720043 & 0.370149 \\
\hline 7 & 6 & 0 & -0.843759 & 3.115005 & -0.149360 \\
\hline 8 & 6 & 0 & 1.911638 & -0.154804 & -1.173195 \\
\hline 9 & 6 & 0 & 3.324761 & -0.441375 & -0.748888 \\
\hline 10 & 7 & 0 & 4. 246517 & 0.581066 & -0.758024 \\
\hline 11 & 6 & 0 & 4.014877 & 1.955208 & -1.171645 \\
\hline 12 & 6 & 0 & 1.140867 & -1.301673 & -1.749696 \\
\hline 13 & 7 & 0 & -2.181279 & -0.583842 & -0.331550 \\
\hline 14 & 6 & 0 & -3.413422 & 0.140390 & 0.061455 \\
\hline 15 & 6 & 0 & -2.260186 & -1.797680 & -1.190796 \\
\hline 16 & 6 & 0 & -0.440836 & 1.583064 & 1.813484 \\
\hline 17 & 6 & 0 & -0.667596 & -2.180288 & 2.142694 \\
\hline 18 & 8 & 0 & 3.656872 & -1.583939 & -0.420478 \\
\hline 19 & 6 & 0 & 5.640432 & 0.262560 & -0.473648 \\
\hline 20 & 1 & 0 & 1.728392 & 0.799446 & -1.655181 \\
\hline 21 & 1 & 0 & 1.639339 & -2.264926 & -1.745000 \\
\hline 22 & 1 & 0 & 0.549209 & -1.095719 & -2.639217 \\
\hline 23 & 1 & 0 & 2.206990 & -1.962500 & 3.238848 \\
\hline 24 & 1 & 0 & 1.973702 & -0.281373 & 2.758274 \\
\hline 25 & 1 & 0 & 3.209233 & -1.271797 & 1.952963 \\
\hline 26 & 1 & 0 & -0.401339 & -2.858247 & 2.965497 \\
\hline 27 & 1 & 0 & -1.454172 & -2.660522 & 1.558186 \\
\hline 28 & 1 & 0 & -1.097008 & -1.279617 & 2.588919 \\
\hline 29 & 1 & 0 & 0.643585 & -4.066527 & -0.142111 \\
\hline 30 & 1 & 0 & 1.438463 & -4.320731 & 1.414497 \\
\hline 31 & 1 & 0 & 2.365162 & -3.659489 & 0.053256 \\
\hline 32 & 6 & 0 & -1.566641 & 2.099904 & 2.727910 \\
\hline 33 & 6 & 0 & 0.904075 & 2.226106 & 2.198900 \\
\hline 34 & 1 & 0 & -0.353535 & 0.511621 & 1.992590 \\
\hline 35 & 6 & 0 & -2.152110 & 3.401980 & -0.901191 \\
\hline 36 & 6 & 0 & 0.365392 & 3.545942 & -0.995684 \\
\hline 37 & 1 & 0 & -0.851251 & 3.752170 & 0.741005 \\
\hline 38 & 6 & 0 & -3.253171 & -2.860612 & -0.692538 \\
\hline 39 & 1 & 0 & -1.269577 & -2.256976 & -1.105110 \\
\hline 40 & 6 & 0 & -2.493732 & -1.561607 & -2.704934 \\
\hline 41 & 6 & 0 & -4.132033 & -0.494116 & 1.269858 \\
\hline 42 & 6 & 0 & -4.424087 & 0.457620 & -1.059691 \\
\hline 43 & 1 & 0 & -3.047420 & 1.104477 & 0.419763 \\
\hline 44 & 1 & 0 & 6.267544 & 0.461633 & -1.353674 \\
\hline 45 & 1 & 0 & 5.709768 & -0.790533 & -0.208564 \\
\hline 46 & 1 & 0 & 6.008159 & 0.878359 & 0.357081 \\
\hline 47 & 1 & 0 & 4.393557 & 2.141412 & -2.187685 \\
\hline 48 & 1 & 0 & 4.543920 & 2.630591 & -0.488227 \\
\hline 49 & 1 & 0 & 2.959644 & 2. 212849 & -1.138349 \\
\hline 50 & 1 & 0 & -1.306404 & 1.922766 & 3.778446 \\
\hline 51 & 1 & 0 & -1.727777 & 3.178186 & 2.612153 \\
\hline 52 & 1 & 0 & -2.511747 & 1.590299 & 2.523378 \\
\hline
\end{tabular}




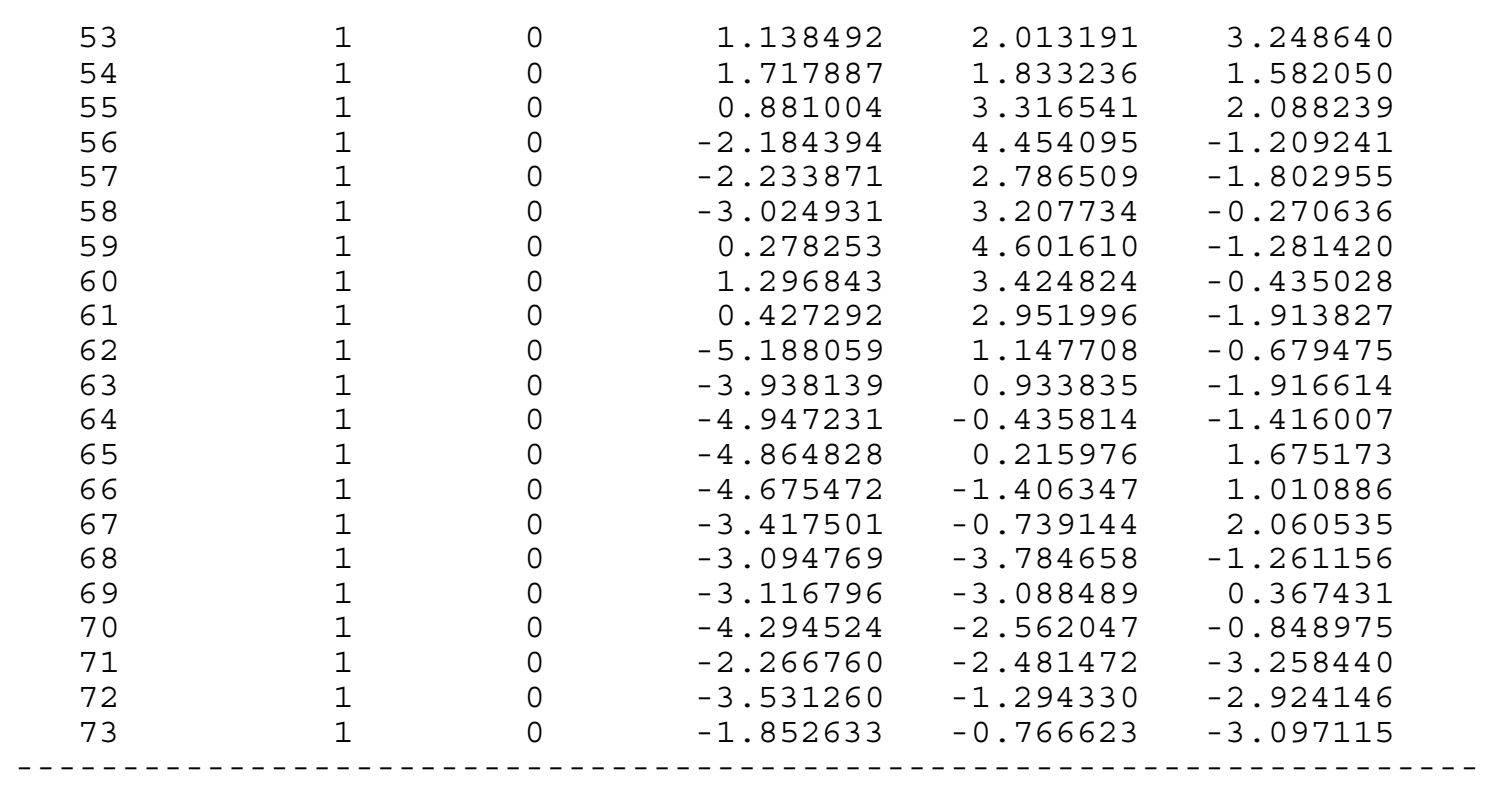

$3 a a$

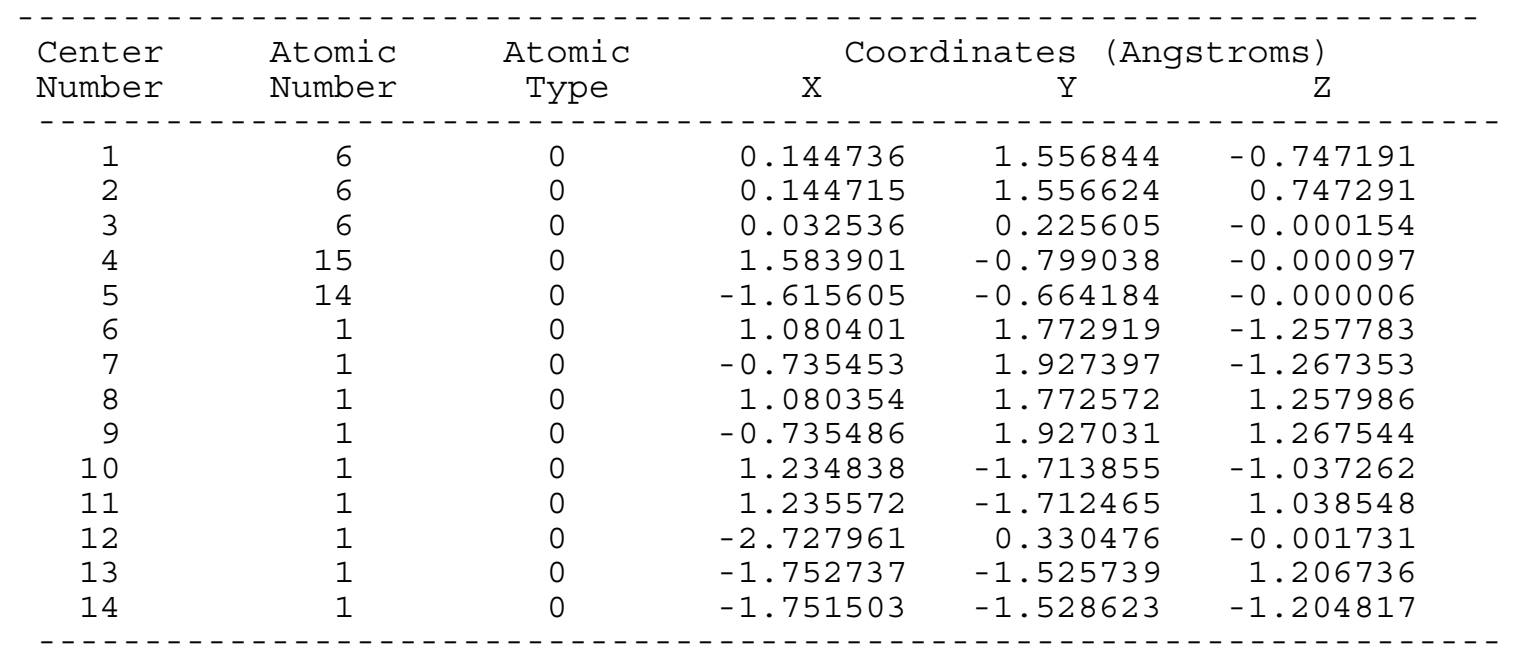

$3 \mathrm{ba}$

\begin{tabular}{|c|c|c|c|c|c|}
\hline Center & Atomic & Atomic & \multicolumn{3}{|c|}{ Coordinates (Angstroms) } \\
\hline Number & Number & Type & $\mathrm{X}$ & $\mathrm{Y}$ & Z \\
\hline------ & & & --------- & --------- & --------- \\
\hline 1 & 6 & 0 & 0.600126 & -0.301812 & -0.015604 \\
\hline 2 & 14 & 0 & 1.335084 & 1.421225 & 0.042789 \\
\hline 3 & 15 & 0 & -1.239377 & -0.557744 & -0.034934 \\
\hline 4 & 6 & 0 & 1.368676 & -1.404929 & 0.717713 \\
\hline 5 & 6 & 0 & 1.389558 & -1.365871 & -0.777337 \\
\hline 6 & 7 & 0 & -1.623587 & 0.404143 & -1.412491 \\
\hline 7 & 7 & 0 & -1.772194 & 0.334951 & 1.350418 \\
\hline 8 & 1 & 0 & 2.821062 & 1.325271 & 0.056874 \\
\hline 9 & 1 & 0 & 0.912699 & 2.156556 & 1.271977 \\
\hline 10 & 1 & 0 & 0.926694 & 2.253866 & -1.122737 \\
\hline 11 & 1 & 0 & 0.784094 & -2.187026 & 1.198104 \\
\hline 12 & 1 & 0 & 2.273549 & -1.138456 & 1.259400 \\
\hline 13 & 1 & 0 & 0.817240 & -2.116569 & -1.317228 \\
\hline 14 & 1 & 0 & 2.309208 & -1.071836 & -1.278025 \\
\hline 15 & 1 & 0 & -2.587662 & 0.276307 & -1.705635 \\
\hline
\end{tabular}




\begin{tabular}{|c|c|c|c|c|c|}
\hline 16 & 1 & 0 & -1.415176 & 1.400118 & -1.375923 \\
\hline 17 & 1 & 0 & -2.123219 & -0.253165 & 2.097956 \\
\hline 18 & 1 & 0 & -1.198691 & 1.085963 & 1.726069 \\
\hline
\end{tabular}

\section{cis $-3 b b$}

\begin{tabular}{|c|c|c|c|c|c|}
\hline \multirow{3}{*}{$\begin{array}{l}\text { Center } \\
\text { Number }\end{array}$} & \multirow{2}{*}{$\begin{array}{l}\text { Atomic } \\
\text { Number }\end{array}$} & Atomic & \multicolumn{3}{|c|}{ Coordinates (Angstroms) } \\
\hline & & Type & $\mathrm{X}$ & $\mathrm{Y}$ & $\mathrm{Z}$ \\
\hline & & & -------- & -------- & -------- \\
\hline 1 & 6 & 0 & 0.834067 & -0.530511 & -0.289638 \\
\hline 2 & 15 & 0 & 0.701478 & 1.332781 & -0.449007 \\
\hline 3 & 7 & 0 & 2.396124 & 1.666884 & -0.189736 \\
\hline 4 & 14 & 0 & 2.485225 & -1.187590 & 0.359462 \\
\hline 5 & 7 & 0 & 0.009819 & 1.753658 & 1.083219 \\
\hline 6 & 6 & 0 & 0.174496 & -1.381278 & -1.362865 \\
\hline 7 & 6 & 0 & -0.417706 & -1.386860 & 0.016094 \\
\hline 8 & 6 & 0 & -1.738049 & -0.762596 & 0.327378 \\
\hline 9 & 8 & 0 & -2.327170 & -0.921886 & 1.377424 \\
\hline 10 & 8 & 0 & -2.215557 & 0.005745 & -0.679941 \\
\hline 11 & 6 & 0 & -3.463932 & 0.662569 & -0.413903 \\
\hline 12 & 1 & 0 & 2.625405 & 1.860914 & 0.783539 \\
\hline 13 & 1 & 0 & 2.714690 & 2.445924 & -0.759204 \\
\hline 14 & 1 & 0 & 2.254176 & -2.638995 & 0.638819 \\
\hline 15 & 1 & 0 & 3.584849 & -1.104448 & -0.631431 \\
\hline 16 & 1 & 0 & 2.892909 & -0.559085 & 1.644940 \\
\hline 17 & 1 & 0 & -0.104789 & 1.022671 & 1.779868 \\
\hline 18 & 1 & 0 & -0.837315 & 2.305885 & 1.014594 \\
\hline 19 & 1 & 0 & -0.384447 & -0.863819 & -2.137369 \\
\hline 20 & 1 & 0 & 0.672335 & -2.289344 & -1.697347 \\
\hline 21 & 1 & 0 & -0.252872 & -2.256588 & 0.645722 \\
\hline 22 & 1 & 0 & -3.696194 & 1.226333 & -1.317588 \\
\hline 23 & 1 & 0 & -4.245857 & -0.071361 & -0.203433 \\
\hline 24 & 1 & 0 & -3.371251 & 1.333846 & 0.444883 \\
\hline
\end{tabular}

\section{trans-3bb}

\begin{tabular}{|c|c|c|c|c|c|}
\hline Center & Atomic & Atomic & \multicolumn{3}{|c|}{ Coordinates (Angstroms) } \\
\hline Number & Number & Type & $\mathrm{X}$ & $\mathrm{Y}$ & Z \\
\hline 1 & 6 & 0 & -0.533713 & -0003248 & -0368967 \\
\hline$\frac{1}{2}$ & 15 & 0 & -2.206361 & -0.00348 & -0.300901 \\
\hline 2 & $\begin{array}{r}7 \\
7\end{array}$ & 0 & & & 0.023488 \\
\hline 3 & & 0 & -2.475586 & -0.381498 & 1.690530 \\
\hline 4 & 14 & 0 & -0.172470 & 1.751750 & 0.231234 \\
\hline 5 & 7 & 0 & -3.188918 & 0.365402 & -0.842765 \\
\hline 6 & 6 & 0 & 0.043953 & -0.488609 & -1.679017 \\
\hline 7 & 6 & 0 & 0.566540 & -1.088586 & -0.398033 \\
\hline 8 & 6 & 0 & 1.956651 & -0.840790 & 0.067678 \\
\hline 9 & 8 & 0 & 2.639640 & -1.650088 & 0.657618 \\
\hline 10 & 8 & 0 & 2.391623 & 0.408711 & -0.245726 \\
\hline 11 & 6 & 0 & 3.725589 & 0.725348 & 0.185511 \\
\hline 12 & 1 & 0 & -2.232406 & -1.132759 & 2.327588 \\
\hline 13 & 1 & 0 & -2.204067 & 0.521587 & 2.072802 \\
\hline 14 & 1 & 0 & 0.556741 & 1.787504 & 1.527058 \\
\hline 15 & 1 & 0 & 0.534381 & 2.582883 & -0.774504 \\
\hline 16 & 1 & 0 & -1.488708 & 2.413605 & 0.490698 \\
\hline 17 & 1 & 0 & -4.142867 & 0.032602 & -0.945535 \\
\hline 18 & 1 & 0 & -3.199399 & 1.331087 & -0.521057 \\
\hline 19 & 1 & 0 & -0.571814 & -1.130936 & -2.305096 \\
\hline 20 & 1 & 0 & 0.703492 & 0.176705 & -2.228476 \\
\hline 21 & 1 & 0 & 0.248136 & -2.100413 & -0.162246 \\
\hline 22 & 1 & 0 & 3.899216 & 1.753827 & -0.131421 \\
\hline 23 & 1 & 0 & 3.808866 & 0.636508 & 1.271608 \\
\hline
\end{tabular}




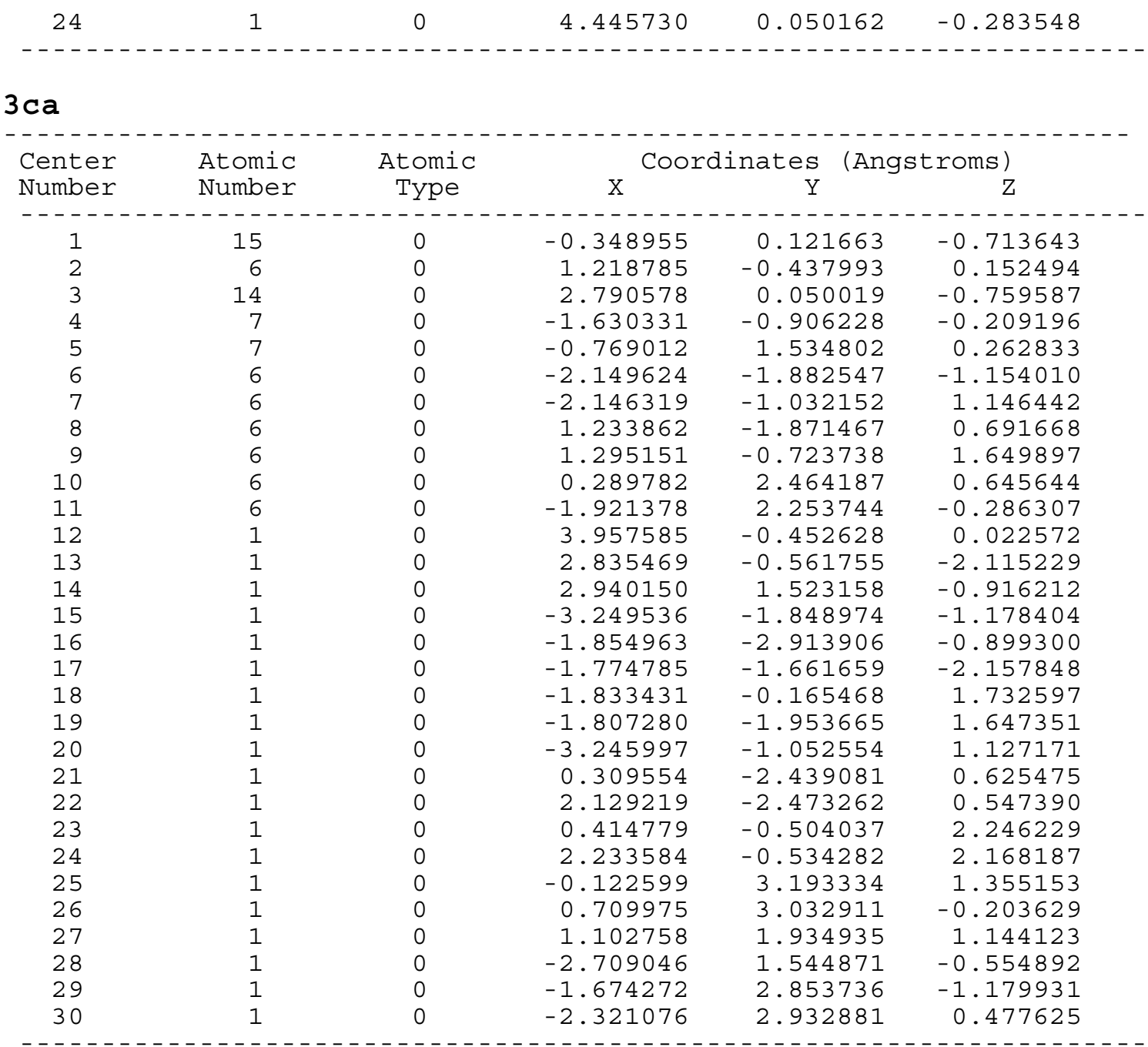

\section{$\mathrm{cis}-3 \mathrm{cb}$}

\begin{tabular}{|c|c|c|c|c|c|}
\hline Center & Atomic & Atomic & \multicolumn{3}{|c|}{ Coordinates (Angstroms) } \\
\hline Number & Number & Type & $\mathrm{X}$ & $\mathrm{Y}$ & $\mathrm{Z}$ \\
\hline------ & & & -------- & -------- & -------- \\
\hline 1 & 15 & 0 & -0.657357 & 0.394163 & 0.579318 \\
\hline 2 & 6 & 0 & -0.007717 & -0.933685 & -0.581330 \\
\hline 3 & 14 & 0 & -0.804612 & -2.628936 & -0.319039 \\
\hline 4 & 1 & 0 & -0.724707 & -3.068200 & 1.097248 \\
\hline 5 & 1 & 0 & 0.014259 & -3.587620 & -1.126406 \\
\hline 6 & 1 & 0 & -2.197401 & -2.751008 & -0.819340 \\
\hline 7 & 6 & 0 & 0.683903 & -0.638136 & -1.891857 \\
\hline 8 & 6 & 0 & 1.540967 & -1.058398 & -0.735202 \\
\hline 9 & 1 & 0 & 0.578785 & -1.332959 & -2.722798 \\
\hline 10 & 1 & 0 & 0.774453 & 0.405922 & -2.176074 \\
\hline 11 & 1 & 0 & 1.946723 & -2.065511 & -0.730706 \\
\hline 12 & 6 & 0 & 2.442958 & -0.051776 & -0.109926 \\
\hline 13 & 8 & 0 & 2.321424 & 1.156446 & -0.168714 \\
\hline 14 & 8 & 0 & 3.449691 & -0.664259 & 0.554092 \\
\hline 15 & 6 & 0 & 4.373137 & 0.215828 & 1.214006 \\
\hline 16 & 1 & 0 & 3.856645 & 0.819458 & 1.965100 \\
\hline 17 & 1 & 0 & 5.110695 & -0.434589 & 1.685214 \\
\hline 18 & 1 & 0 & 4.853226 & 0.882442 & 0.492463 \\
\hline 19 & 7 & 0 & -2.233581 & -0.334926 & 0.859512 \\
\hline
\end{tabular}




\begin{tabular}{|c|c|c|c|c|c|}
\hline 20 & 7 & 0 & -0.858470 & 1.792449 & -0.384157 \\
\hline 21 & 6 & 0 & -1.408542 & 1.904901 & -1.726290 \\
\hline 22 & 1 & 0 & -0.693847 & 2.406487 & -2.397377 \\
\hline 23 & 1 & 0 & -1.628326 & 0.922446 & -2.145970 \\
\hline 24 & 1 & 0 & -2.337923 & 2.496121 & -1.733126 \\
\hline 25 & 6 & 0 & -0.373828 & 3.062615 & 0.144566 \\
\hline 26 & 1 & 0 & -1.186243 & 3.803950 & 0.202666 \\
\hline 27 & 1 & 0 & 0.036534 & 2.917992 & 1. 146172 \\
\hline 28 & 1 & 0 & 0.423733 & 3.473091 & -0.491509 \\
\hline 29 & 6 & 0 & -3.312627 & -0.226481 & -0.119367 \\
\hline 30 & 1 & 0 & -3.748307 & 0.785567 & -0.156482 \\
\hline 31 & 1 & 0 & -2.957473 & -0.487015 & -1.118469 \\
\hline 32 & 1 & 0 & -4.113644 & -0.931286 & 0.138746 \\
\hline 33 & 6 & 0 & -2.736935 & -0.141218 & 2.222252 \\
\hline 34 & 1 & 0 & -3.474984 & -0.920182 & 2.453380 \\
\hline 35 & 1 & 0 & -1.915187 & -0.223233 & 2.937696 \\
\hline 36 & 1 & 0 & -3.224559 & 0.838718 & 2.364733 \\
\hline
\end{tabular}

\section{trans $-3 \mathrm{cb}$}

\begin{tabular}{|c|c|c|c|c|c|}
\hline \multirow{2}{*}{$\begin{array}{l}\text { Center } \\
\text { Number }\end{array}$} & \multirow{2}{*}{$\begin{array}{l}\text { Atomic } \\
\text { Number }\end{array}$} & \multirow{2}{*}{$\begin{array}{c}\text { Atomic } \\
\text { Type }\end{array}$} & \multicolumn{3}{|c|}{ Coordinates (Angstroms) } \\
\hline & & & $\mathrm{X}$ & $\mathrm{Y}$ & $\mathrm{Z}$ \\
\hline & & & $19000-1$ & --------- & --------1 \\
\hline 1 & 15 & 0 & 1.679611 & 0.455297 & -0.436683 \\
\hline 2 & 6 & 0 & -0.149182 & 0.249882 & -0.867793 \\
\hline 3 & 14 & 0 & -0.929962 & 1.814217 & -1.627989 \\
\hline 4 & 1 & 0 & -1.781111 & 2.563362 & -0.668771 \\
\hline 5 & 1 & 0 & -1.686219 & 1.511289 & -2.868010 \\
\hline 6 & 1 & 0 & 0.198919 & 2.715007 & -1.998171 \\
\hline 7 & 7 & 0 & 2.341673 & -1.116813 & -0.231082 \\
\hline 8 & 7 & 0 & 1.556916 & 0.905143 & 1.266680 \\
\hline 9 & 6 & 0 & 2.860623 & 1.260412 & 1.833761 \\
\hline 10 & 1 & 0 & 2.793934 & 1.244969 & 2.928993 \\
\hline 11 & 1 & 0 & 3.612048 & 0.526342 & 1.530730 \\
\hline 12 & 1 & 0 & 3.210482 & 2.261786 & 1.527472 \\
\hline 13 & 6 & 0 & 0.531475 & 1.860370 & 1.675887 \\
\hline 14 & 1 & 0 & 0.702918 & 2.885410 & 1.300152 \\
\hline 15 & 1 & 0 & -0.455248 & 1.531148 & 1.348388 \\
\hline 16 & 1 & 0 & 0.517430 & 1.913275 & 2.772124 \\
\hline 17 & 6 & 0 & 3.261896 & -1.616055 & -1.242079 \\
\hline 18 & 1 & 0 & 2.835161 & -2.452863 & -1.818188 \\
\hline 19 & 1 & 0 & 3.512158 & -0.814340 & -1.943096 \\
\hline 20 & 1 & 0 & 4.193161 & -1.974527 & -0.777111 \\
\hline 21 & 6 & 0 & 2.023817 & -2.066892 & 0.825552 \\
\hline 22 & 1 & 0 & 1.488557 & -1.551721 & 1.626085 \\
\hline 23 & 1 & 0 & 1.417713 & -2.913204 & 0.464017 \\
\hline 24 & 1 & 0 & 2.950980 & -2.481973 & 1.247995 \\
\hline 25 & 6 & 0 & -0.503590 & -1.071011 & -1.516431 \\
\hline 26 & 6 & 0 & -1.011364 & -0.787996 & -0.124260 \\
\hline 27 & 6 & 0 & -2.465292 & -0.532712 & 0.042252 \\
\hline 28 & 8 & 0 & -3.168798 & 0.060321 & -0.755803 \\
\hline 29 & 8 & 0 & -2.926501 & -1.040623 & 1.204892 \\
\hline 30 & 6 & 0 & -4.328471 & -0.840199 & 1.452319 \\
\hline 31 & 1 & 0 & -1.239158 & -1.069280 & -2.316533 \\
\hline 32 & 1 & 0 & 0.273702 & -1.825792 & -1.605828 \\
\hline 33 & 1 & 0 & -0.552436 & -1.316406 & 0.702487 \\
\hline 34 & 1 & 0 & -4.928036 & -1.320409 & 0.674509 \\
\hline 35 & 1 & 0 & -4.521441 & -1.297929 & 2.422947 \\
\hline 36 & 1 & 0 & -4.565408 & 0.226669 & 1.474754 \\
\hline
\end{tabular}

\section{cis-3cc}




\begin{tabular}{|c|c|c|c|c|c|}
\hline Center & Atomic & Atomic & \multicolumn{3}{|c|}{ Coordinates (Angstroms) } \\
\hline Number & Number & Type & $\mathrm{X}$ & $\mathrm{Y}$ & Z \\
\hline & & & -------- & -------- & -------- \\
\hline 1 & 15 & 0 & -0.862737 & -0.345552 & -0.660243 \\
\hline 2 & 6 & 0 & -0.358968 & 1.011746 & 0.527385 \\
\hline 3 & 14 & 0 & -1.232082 & 2.627935 & 0.076281 \\
\hline 4 & 1 & 0 & -1.241102 & 2.867293 & -1.391573 \\
\hline 5 & 1 & 0 & -0.445625 & 3.734612 & 0.706256 \\
\hline 6 & 1 & 0 & -2.614859 & 2.727121 & 0.609498 \\
\hline 7 & 6 & 0 & 0.132261 & 0.879805 & 1.963103 \\
\hline 8 & 6 & 0 & 1.137054 & 1.172308 & 0.898739 \\
\hline 9 & 1 & 0 & -0.110324 & 1.672485 & 2.669045 \\
\hline 10 & 1 & 0 & 0.203480 & -0.107187 & 2.403825 \\
\hline 11 & 1 & 0 & 1.529076 & 2.186093 & 0.849470 \\
\hline 12 & 6 & 0 & 2.157407 & 0.096457 & 0.582807 \\
\hline 13 & 8 & 0 & 2.265350 & -0.916318 & 1.274934 \\
\hline 14 & 7 & 0 & 2.983656 & 0.333687 & -0.488286 \\
\hline 15 & 6 & 0 & 2.836501 & 1.437215 & -1.423638 \\
\hline 16 & 6 & 0 & 4.085535 & -0.579319 & -0.752410 \\
\hline 17 & 1 & 0 & 1.845015 & 1.881747 & -1.348702 \\
\hline 18 & 1 & 0 & 2.954784 & 1.062527 & -2.447477 \\
\hline 19 & 7 & 0 & -2.576217 & -0.029525 & -0.603945 \\
\hline 20 & 7 & 0 & -0.537037 & -1.860062 & 0.061228 \\
\hline 21 & 6 & 0 & -0.832868 & -2.313895 & 1.414674 \\
\hline 22 & 1 & 0 & 0.093236 & -2.454819 & 1.990041 \\
\hline 23 & 1 & 0 & -1.462020 & -1.595726 & 1.940750 \\
\hline 24 & 1 & 0 & -1.369330 & -3.274901 & 1.386189 \\
\hline 25 & 6 & 0 & 0.306230 & -2.800268 & -0.670994 \\
\hline 26 & 1 & 0 & -0.180970 & -3.785307 & -0.736983 \\
\hline 27 & 1 & 0 & 0.478278 & -2.435125 & -1.687125 \\
\hline 28 & 1 & 0 & 1.278268 & -2.924991 & -0.173317 \\
\hline 29 & 6 & 0 & -3.370530 & -0.153566 & 0.616030 \\
\hline 30 & 1 & 0 & -3.654031 & -1.197057 & 0.831495 \\
\hline 31 & 1 & 0 & -2.820144 & 0.242343 & 1.472903 \\
\hline 32 & 1 & 0 & -4.295114 & 0.430187 & 0.513779 \\
\hline 33 & $\overline{6}$ & 0 & -3.318524 & -0.485069 & -1.778957 \\
\hline 34 & 1 & 0 & -2.711938 & -0.344438 & -2.677601 \\
\hline 35 & 1 & 0 & -3.606565 & -1.548471 & -1.716689 \\
\hline 36 & 1 & 0 & -4.237605 & 0.106046 & -1.885416 \\
\hline 37 & 1 & 0 & 3.594974 & 2.216316 & -1.257734 \\
\hline 38 & 1 & 0 & 4.136849 & -1.303928 & 0.058877 \\
\hline 39 & 1 & 0 & 5.030863 & -0.024342 & -0.813897 \\
\hline 40 & 1 & 0 & 3.934814 & -1.108936 & -1.702792 \\
\hline
\end{tabular}

\section{trans-3cc}

\begin{tabular}{|c|c|c|c|c|c|}
\hline Center & Atomic & Atomic & \multicolumn{3}{|c|}{ Coordinates (Angstroms) } \\
\hline Number & Number & Type & $\mathrm{X}$ & $\mathrm{Y}$ & Z \\
\hline & & & - & & \\
\hline 1 & 15 & 0 & 1.885191 & 0.603753 & 0.112460 \\
\hline 2 & 6 & 0 & 0.097875 & 0.961380 & -0.366867 \\
\hline 3 & 14 & 0 & -0.573508 & 2.601585 & 0.340428 \\
\hline 4 & 1 & 0 & -1.496404 & 2.420956 & 1.489694 \\
\hline 5 & 1 & 0 & -1.168061 & 3.476801 & -0.698686 \\
\hline 6 & 1 & 0 & 0.617231 & 3.323981 & 0.882087 \\
\hline 7 & 7 & 0 & 2.498314 & -0.575854 & -0.980500 \\
\hline 8 & 7 & 0 & 1.627629 & -0.463457 & 1.503937 \\
\hline 9 & 6 & 0 & 2.893386 & -0.840675 & 2.140506 \\
\hline 10 & 1 & 0 & 2.718722 & -1.686616 & 2.817568 \\
\hline 11 & 1 & 0 & 3.612851 & -1.156227 & 1.380570 \\
\hline 12 & 1 & 0 & 3.345562 & -0.022172 & 2.727586 \\
\hline 13 & 6 & 0 & 0.652877 & -0.042673 & 2.508534 \\
\hline 14 & 1 & 0 & 0.954440 & 0.861216 & 3.067865 \\
\hline
\end{tabular}




\begin{tabular}{|c|c|c|c|c|c|}
\hline 15 & 1 & 0 & -0.319084 & 0.144404 & 2.049725 \\
\hline 16 & 1 & 0 & 0.528104 & -0.851629 & 3.240144 \\
\hline 17 & 6 & 0 & 3.466804 & -0.148373 & -1.978843 \\
\hline 18 & 1 & 0 & 3.045130 & -0.139152 & -2.997246 \\
\hline 19 & 1 & 0 & 3.814438 & 0.862640 & -1.747067 \\
\hline 20 & 1 & 0 & 4.337495 & -0.821912 & -1.986838 \\
\hline 21 & 6 & 0 & 2.055281 & -1.955071 & -1.116239 \\
\hline 22 & 1 & 0 & 1.487832 & -2.240731 & -0.227489 \\
\hline 23 & 1 & 0 & 1.436244 & -2.118346 & -2.013971 \\
\hline 24 & 1 & 0 & 2.926610 & -2.622441 & -1.192446 \\
\hline 25 & 6 & 0 & -0.262125 & 0.648814 & -1.804334 \\
\hline 26 & 6 & 0 & -0.863643 & -0.193633 & -0.708341 \\
\hline 27 & 6 & 0 & -2.320227 & 0.015164 & -0.426125 \\
\hline 28 & 8 & 0 & -2.814436 & 1.137547 & -0.582392 \\
\hline 29 & 7 & 0 & -3.067874 & -1.045990 & 0.016786 \\
\hline 30 & 6 & 0 & -4.505315 & -0.864231 & 0.185410 \\
\hline 31 & 1 & 0 & -0.936073 & 1.327723 & -2.318821 \\
\hline 32 & 1 & 0 & 0.490020 & 0.175657 & -2.430846 \\
\hline 33 & 1 & 0 & -0.461070 & -1.190752 & -0.596827 \\
\hline 34 & 6 & 0 & -2.567616 & -2.407055 & 0.168771 \\
\hline 35 & 1 & 0 & -5.064293 & -1.433016 & -0.570239 \\
\hline 36 & 1 & 0 & -4.731886 & 0.195194 & 0.077994 \\
\hline 37 & 1 & 0 & -4.814837 & -1.209265 & 1.179233 \\
\hline 38 & 1 & 0 & -2.555630 & -2.959784 & -0.781480 \\
\hline 39 & 1 & 0 & -3.226279 & -2.940608 & 0.860137 \\
\hline 40 & 1 & 0 & -1.564592 & -2.419553 & 0.599212 \\
\hline
\end{tabular}

cis-3cd

\begin{tabular}{|c|c|c|c|c|c|}
\hline Center & Atomic & Atomic & \multicolumn{3}{|c|}{ Coordinates (Angstroms) } \\
\hline Number & Number & Type & $\mathrm{X}$ & $\mathrm{Y}$ & Z \\
\hline------ & & & --------- & --------- & -------- \\
\hline 1 & 15 & 0 & 0.911098 & -0.431729 & -0.590717 \\
\hline 2 & 6 & 0 & 0.695249 & 1.137995 & 0.394613 \\
\hline 3 & 14 & 0 & 1.898964 & 2.492691 & -0.127543 \\
\hline 4 & 7 & 0 & 2.652314 & -0.463099 & -0.627305 \\
\hline 5 & 7 & 0 & 0.297776 & -1.678053 & 0.431044 \\
\hline 6 & 6 & 0 & 3.242400 & -1.147520 & -1.776641 \\
\hline 7 & 6 & 0 & 3.503543 & -0.554930 & 0.555158 \\
\hline 8 & 1 & 0 & 3.403971 & -2.224487 & -1.597097 \\
\hline 9 & 1 & 0 & 3.074394 & 0.013666 & 1.383179 \\
\hline 10 & 6 & 0 & 0.070007 & 1.215458 & 1.785774 \\
\hline 11 & 6 & 0 & -0.754261 & 1.646753 & 0.616635 \\
\hline 12 & 1 & 0 & -0.201585 & 0.276785 & 2.257214 \\
\hline 13 & 1 & 0 & 0.427412 & 1.971423 & 2.482914 \\
\hline 14 & 1 & 0 & -0.863705 & 2.725346 & 0.496185 \\
\hline 15 & 6 & 0 & -0.535131 & -2.701691 & -0.191771 \\
\hline 16 & 6 & 0 & 0.812670 & -2.087928 & 1.729343 \\
\hline 17 & 1 & 0 & -1.414425 & -2.909524 & 0.434538 \\
\hline 18 & 1 & 0 & 1.392854 & -1.289260 & 2.192682 \\
\hline 19 & 1 & 0 & 1.376327 & 3.777621 & 0.430718 \\
\hline 20 & 1 & 0 & 3.279744 & 2.330253 & 0.398518 \\
\hline 21 & 1 & 0 & 4.217123 & -0.700287 & -2.014163 \\
\hline 22 & 1 & 0 & 2.591387 & -1.037061 & -2.647710 \\
\hline 23 & 1 & 0 & 0.009896 & -3.650053 & -0.335752 \\
\hline 24 & 1 & 0 & -0.889447 & -2.353292 & -1.164508 \\
\hline 25 & 1 & 0 & 4.490368 & -0.125998 & 0.334296 \\
\hline 26 & 1 & 0 & 3.656533 & -1.595547 & 0.885907 \\
\hline 27 & 1 & 0 & 1.953566 & 2.634299 & -1.607148 \\
\hline 28 & 1 & 0 & 1.458617 & -2.978674 & 1.652687 \\
\hline 29 & 1 & 0 & -0.016457 & -2.342713 & 2.407274 \\
\hline 30 & 6 & 0 & -1.971516 & 0.895191 & 0.158386 \\
\hline 31 & 6 & 0 & -2.342311 & 0.930134 & -1.194744 \\
\hline
\end{tabular}




$\begin{array}{rrrrrr}32 & 6 & 0 & -3.498034 & 0.287822 & -1.638320 \\ 33 & 6 & 0 & -4.309099 & -0.400852 & -0.733530 \\ 34 & 6 & 0 & -3.955501 & -0.437727 & 0.615985 \\ 35 & 6 & 0 & -2.798027 & 0.207262 & 1.056169 \\ 36 & 1 & 0 & -1.712386 & 1.460829 & -1.903962 \\ 37 & 1 & 0 & -3.764659 & 0.324212 & -2.691493 \\ 38 & 1 & 0 & -5.210336 & -0.901608 & -1.077452 \\ 39 & 1 & 0 & -4.584073 & -0.963742 & 1.330252 \\ 40 & 1 & 0 & -2.537641 & 0.184330 & 2.110781 \\ - & & & & \end{array}$

\section{trans-3cd}

\begin{tabular}{|c|c|c|c|c|c|}
\hline \multirow{2}{*}{$\begin{array}{l}\text { Center } \\
\text { Number }\end{array}$} & \multirow{2}{*}{$\begin{array}{l}\text { Atomic } \\
\text { Number }\end{array}$} & \multirow{2}{*}{$\begin{array}{c}\text { Atomic } \\
\text { Type }\end{array}$} & \multicolumn{3}{|c|}{ Coordinates (Angstroms) } \\
\hline & & & $\mathrm{X}$ & $\mathrm{Y}$ & $\mathrm{Z}$ \\
\hline & & & & 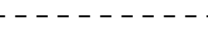 & ---------- \\
\hline 1 & 15 & 0 & -1.620719 & 0.144424 & -0.706707 \\
\hline 2 & 6 & 0 & -0.158408 & -0.331002 & 0.346543 \\
\hline 3 & 14 & 0 & 0.329494 & -2.143473 & 0.120484 \\
\hline 4 & 7 & 0 & -2.724945 & -1.118783 & -0.285239 \\
\hline 5 & 7 & 0 & -2.127064 & 1.666118 & -0.033749 \\
\hline 6 & 6 & 0 & -3.755853 & -1.435622 & -1.269039 \\
\hline 7 & 6 & 0 & -3.108162 & -1.499886 & 1.069209 \\
\hline 8 & 1 & 0 & -4.701609 & -0.898203 & -1.083703 \\
\hline 9 & 1 & 0 & -2.294524 & -1.296763 & 1.768442 \\
\hline 10 & 6 & 0 & 0.258501 & 0.350153 & 1.653631 \\
\hline 11 & 6 & 0 & 0.948310 & 0.740697 & 0.386106 \\
\hline 12 & 1 & 0 & 0.605236 & 1.696037 & -0.014939 \\
\hline 13 & 1 & 0 & -0.400924 & 1.080695 & 2.109897 \\
\hline 14 & 1 & 0 & 0.804715 & -0.255493 & 2.375057 \\
\hline 15 & 6 & 0 & -2.506696 & 2.699649 & -0.994373 \\
\hline 16 & 6 & 0 & -2.812451 & 1.832409 & 1.242804 \\
\hline 17 & 1 & 0 & -2.208987 & 3.690161 & -0.621680 \\
\hline 18 & 1 & 0 & -2.522071 & 1.053686 & 1.948403 \\
\hline 19 & 1 & 0 & 1.674951 & -2.375217 & 0.710524 \\
\hline 20 & 1 & 0 & -0.614546 & -3.049914 & 0.826679 \\
\hline 21 & 1 & 0 & -3.975716 & -2.512029 & -1.247033 \\
\hline 22 & 1 & 0 & -3.403393 & -1.177640 & -2.271404 \\
\hline 23 & 1 & 0 & -3.594932 & 2.726461 & -1.178082 \\
\hline 24 & 1 & 0 & -2.002538 & 2.526480 & -1.948290 \\
\hline 25 & 1 & 0 & -3.318221 & -2.577683 & 1.105837 \\
\hline 26 & 1 & 0 & -4.011188 & -0.971042 & 1.415997 \\
\hline 27 & 1 & 0 & 0.363770 & -2.520628 & -1.317912 \\
\hline 28 & 1 & 0 & -3.909634 & 1.797899 & 1.130607 \\
\hline 29 & 1 & 0 & -2.558529 & 2.805889 & 1.687480 \\
\hline 30 & 6 & 0 & 2.395154 & 0.468168 & 0.098760 \\
\hline 31 & 6 & 0 & 3.350710 & 0.391141 & 1.119335 \\
\hline 32 & 6 & 0 & 4.698324 & 0.175270 & 0.824108 \\
\hline 33 & 6 & 0 & 5.113330 & 0.038940 & -0.500776 \\
\hline 34 & 6 & 0 & 4.171491 & 0.123225 & -1.529205 \\
\hline 35 & 6 & 0 & 2.826667 & 0.338442 & -1.230928 \\
\hline 36 & 1 & 0 & 3.037420 & 0.506278 & 2.153461 \\
\hline 37 & 1 & 0 & 5.423996 & 0.116735 & 1.631420 \\
\hline 38 & 1 & 0 & 6.161844 & -0.129693 & -0.731588 \\
\hline 39 & 1 & 0 & 4.484758 & 0.020723 & -2.565048 \\
\hline 40 & 1 & 0 & 2.096545 & 0.404665 & -2.034437 \\
\hline
\end{tabular}

\section{cis-3ce}

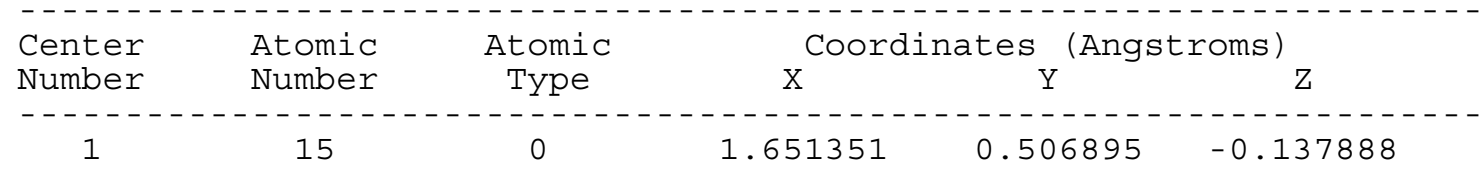




\begin{tabular}{|c|c|c|c|c|c|}
\hline & & & & & \\
\hline 2 & 6 & 0 & -0.025257 & -0.165867 & -0.703383 \\
\hline 3 & 14 & 0 & 0.141312 & -1.087698 & -2.371138 \\
\hline 4 & 1 & 0 & 1.140486 & -0.348868 & -3.187644 \\
\hline 5 & 1 & 0 & -1.140057 & -1.018283 & -3.117894 \\
\hline 6 & 1 & 0 & 0.551208 & -2.507704 & -2.240152 \\
\hline 7 & 6 & 0 & -1.152432 & -0.401399 & 0.290275 \\
\hline 8 & 6 & 0 & -1.268962 & 0.743559 & -0.688699 \\
\hline 9 & 6 & 0 & -2.064216 & -1.574571 & 0.164845 \\
\hline 10 & 1 & 0 & -0.957334 & -0.084967 & 1.308582 \\
\hline 11 & 1 & 0 & -1.951476 & 0.607749 & -1.521933 \\
\hline 12 & 6 & 0 & -1.242959 & 2.140948 & -0.161985 \\
\hline 13 & 8 & 0 & -0.751293 & 2.510923 & 0.885069 \\
\hline 14 & 8 & 0 & -1.857298 & 2.973468 & -1.030937 \\
\hline 15 & 6 & 0 & -1.882695 & 4.357078 & -0.643400 \\
\hline 16 & 1 & 0 & -0.865789 & 4.743460 & -0.536225 \\
\hline 17 & 1 & 0 & -2.407934 & 4.874868 & -1.446231 \\
\hline 18 & 1 & 0 & -2.411648 & 4.480168 & 0.305420 \\
\hline 19 & 7 & 0 & 1.636535 & 0.545573 & 1.571623 \\
\hline 20 & 7 & 0 & 2.585736 & -0.871459 & -0.654957 \\
\hline 21 & 6 & 0 & 2.482582 & -2.192306 & -0.041714 \\
\hline 22 & 1 & 0 & 3.029327 & -2.262276 & 0.913117 \\
\hline 23 & 1 & 0 & 1.436905 & -2.455681 & 0.133730 \\
\hline 24 & 1 & 0 & 2.900037 & -2.944642 & -0.723586 \\
\hline 25 & 6 & 0 & 3.964744 & -0.559463 & -1.031011 \\
\hline 26 & 1 & 0 & 4.330967 & -1.312892 & -1.740722 \\
\hline 27 & 1 & 0 & 4.005321 & 0.418527 & -1.517141 \\
\hline 28 & 1 & 0 & 4.652349 & -0.547179 & -0.167791 \\
\hline 29 & 6 & 0 & 1.275391 & -0.501927 & 2.516103 \\
\hline 30 & 1 & 0 & 0.512379 & -0.142103 & 3.223780 \\
\hline 31 & 1 & 0 & 0.871948 & -1.377524 & 2.007097 \\
\hline 32 & 1 & 0 & 2.147252 & -0.823001 & 3.107869 \\
\hline 33 & 6 & 0 & 2.019535 & 1.791244 & 2.228332 \\
\hline 34 & 1 & 0 & 2.301168 & 2.536314 & 1.481183 \\
\hline 35 & 1 & 0 & 1.184334 & 2.198718 & 2.814040 \\
\hline 36 & 1 & 0 & 2.875859 & 1.631749 & 2.902238 \\
\hline 37 & 8 & 0 & -2.365786 & -2.133328 & -0.871189 \\
\hline 38 & 8 & 0 & -2.513291 & -1.962436 & 1.378627 \\
\hline 39 & 6 & 0 & -3.398752 & -3.095826 & 1.372140 \\
\hline 40 & 1 & 0 & -3.654343 & -3.271910 & 2.417211 \\
\hline 41 & 1 & 0 & -2.900088 & -3.969226 & 0.943784 \\
\hline 42 & 1 & 0 & -4.295943 & -2.877579 & 0.787278 \\
\hline------ & ------ & ----- & -------1 & ------ & ------- \\
\hline trans -3 & & & & & \\
\hline 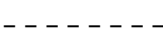 & - & -----6 & ----------- & ----------- & ---------- \\
\hline Center & Atomic & Atomic & Coor & inates (Ang & roms ) \\
\hline Number & Number & Type & $\mathrm{X}$ & $\mathrm{Y}$ & Z \\
\hline------- & ------- & ------- & ---------- & --------- & --------- \\
\hline 1 & 15 & 0 & 1.392265 & -1.034319 & -0.353303 \\
\hline 2 & 6 & 0 & -0.205547 & -0.099107 & -0.714375 \\
\hline 3 & 14 & 0 & -0.968058 & -0.847809 & -2.306264 \\
\hline 4 & 1 & 0 & -1.906482 & -1.964066 & -2.034076 \\
\hline 5 & 1 & 0 & -1.652718 & 0.206212 & -3.097250 \\
\hline 6 & 1 & 0 & 0.156470 & -1.341994 & -3.144591 \\
\hline 7 & 7 & 0 & 1.887917 & -0.745686 & 1.256840 \\
\hline 8 & 7 & 0 & 0.681353 & -2.618419 & -0.446090 \\
\hline 9 & 6 & 0 & 1.614320 & -3.689784 & -0.791266 \\
\hline 10 & 1 & 0 & 2.130494 & -4.106674 & 0.090388 \\
\hline 11 & 1 & 0 & 2.368680 & -3.314590 & -1.487860 \\
\hline 12 & 1 & 0 & 1.069469 & -4.508184 & -1.280042 \\
\hline 13 & 6 & 0 & -0.388663 & -3.092652 & 0.426911 \\
\hline 14 & 1 & 0 & -0.928431 & -3.911112 & -0.067727 \\
\hline 15 & 1 & 0 & -1.107879 & -2.294675 & 0.624769 \\
\hline 16 & 1 & 0 & -0.012881 & -3.474158 & 1.390993 \\
\hline
\end{tabular}




\begin{tabular}{|c|c|c|c|c|c|}
\hline 17 & 6 & 0 & 1.119827 & -0.832816 & 2.491909 \\
\hline 18 & 1 & 0 & 1.629109 & -1.490504 & 3.213097 \\
\hline 19 & 1 & 0 & 0.125552 & -1.242524 & 2.312748 \\
\hline 20 & 1 & 0 & 1.010411 & 0.157582 & 2.958284 \\
\hline 21 & 6 & 0 & 3.228841 & -0.207536 & 1.468187 \\
\hline 22 & 1 & 0 & 3.777991 & -0.192609 & 0.522993 \\
\hline 23 & 1 & 0 & 3.786653 & -0.833971 & 2.181175 \\
\hline 24 & 1 & 0 & 3.185780 & 0.816911 & 1.862223 \\
\hline 25 & 6 & 0 & -0.294098 & 1.431021 & -0.660129 \\
\hline 26 & 6 & 0 & -1.060944 & 0.600681 & 0.340082 \\
\hline 27 & 6 & 0 & -2.548112 & 0.561989 & 0.261857 \\
\hline 28 & 8 & 0 & -3.201134 & 0.521021 & -0.762844 \\
\hline 29 & 8 & 0 & -3.096174 & 0.561862 & 1.495716 \\
\hline 30 & 6 & 0 & -4.533567 & 0.521340 & 1.530678 \\
\hline 31 & 1 & 0 & -0.862798 & 1.885535 & -1.466125 \\
\hline 32 & 6 & 0 & 0.809084 & 2.304458 & -0.149589 \\
\hline 33 & 1 & 0 & -0.661520 & 0.642229 & 1.345661 \\
\hline 34 & 1 & 0 & -4.953256 & 1.402130 & 1.037784 \\
\hline 35 & 1 & 0 & -4.798880 & 0.510094 & 2.588052 \\
\hline 36 & 1 & 0 & -4.904340 & -0.376336 & 1.029157 \\
\hline 37 & 8 & 0 & 1.284898 & 2.290152 & 0.967611 \\
\hline 38 & 8 & 0 & 1.209955 & 3.154499 & -1.119176 \\
\hline 39 & 6 & 0 & 2.269433 & 4.053361 & -0.753069 \\
\hline 40 & 1 & 0 & 2.456508 & 4.659629 & -1.639638 \\
\hline 41 & 1 & 0 & 1.965702 & 4.683115 & 0.087469 \\
\hline 42 & 1 & 0 & 3.166512 & 3.494788 & -0.473132 \\
\hline
\end{tabular}

\section{cis-3cf}

\begin{tabular}{|c|c|c|c|c|c|}
\hline \multirow{3}{*}{$\begin{array}{l}\text { Center } \\
\text { Number }\end{array}$} & \multirow{2}{*}{$\begin{array}{l}\text { Atomic } \\
\text { Number }\end{array}$} & Atomic & \multicolumn{3}{|c|}{ Coordinates (Angstroms) } \\
\hline & & Type & $\mathrm{X}$ & $\mathrm{Y}$ & $\mathrm{Z}$ \\
\hline & & & -------- & --------- & -------- \\
\hline 1 & 15 & 0 & -1.091480 & -0.023409 & -0.836317 \\
\hline 2 & 6 & 0 & -0.364598 & -0.374517 & 0.869842 \\
\hline 3 & 14 & 0 & -1.704594 & -0.723594 & 2.174576 \\
\hline 4 & 1 & 0 & -2.276133 & -2.092491 & 2.106017 \\
\hline 5 & 1 & 0 & -0.912195 & -0.718792 & 3.456510 \\
\hline 6 & 1 & 0 & -2.750868 & 0.311214 & 2.374537 \\
\hline 7 & 7 & 0 & -1.337209 & 1.667630 & -0.821587 \\
\hline 8 & 7 & 0 & -2.680410 & -0.703619 & -0.283688 \\
\hline 9 & 6 & 0 & -3.886353 & 0.048748 & -0.626725 \\
\hline 10 & 1 & 0 & -4.077137 & 0.088677 & -1.713148 \\
\hline 11 & 1 & 0 & -3.819995 & 1.072281 & -0.254515 \\
\hline 12 & 1 & 0 & -4.748815 & -0.435352 & -0.151673 \\
\hline 13 & 6 & 0 & -2.790458 & -2.098027 & -0.741923 \\
\hline 14 & 1 & 0 & -3.580077 & -2.605469 & -0.174361 \\
\hline 15 & 1 & 0 & -1.850039 & -2.630600 & -0.570119 \\
\hline 16 & 1 & 0 & -3.027397 & -2.171150 & -1.815909 \\
\hline 17 & 6 & 0 & -0.943049 & 2.399269 & -2.022345 \\
\hline 18 & 1 & 0 & -1.725322 & 3.118232 & -2.306246 \\
\hline 19 & 1 & 0 & -0.791776 & 1.704681 & -2.851552 \\
\hline 20 & 1 & 0 & -0.004096 & 2.954248 & -1.871275 \\
\hline 21 & 6 & 0 & -1.530227 & 2.531027 & 0.335585 \\
\hline 22 & 1 & 0 & -1.959359 & 1.978625 & 1.171492 \\
\hline 23 & 1 & 0 & -2.223000 & 3.346448 & 0.082529 \\
\hline 24 & 1 & 0 & -0.585983 & 2.989759 & 0.672351 \\
\hline 25 & 6 & 0 & 0.979057 & 0.231500 & 1.321870 \\
\hline 26 & 6 & 0 & 0.867103 & -1.241762 & 1.028155 \\
\hline 27 & 6 & 0 & 1.547778 & -1.918483 & -0.136015 \\
\hline 28 & 8 & 0 & 0.976517 & -2.546028 & -1.000507 \\
\hline 29 & 8 & 0 & 2.887768 & -1.829400 & -0.029475 \\
\hline 30 & 6 & 0 & 3.628906 & -2.466556 & -1.084439 \\
\hline
\end{tabular}




\begin{tabular}{|c|c|c|c|c|c|}
\hline 31 & 1 & 0 & 1.067258 & 0.524054 & 2.363967 \\
\hline 32 & 6 & 0 & 1.759211 & 1.091314 & 0.387139 \\
\hline 33 & 1 & 0 & 0.907752 & -1.875807 & 1.915941 \\
\hline 34 & 1 & 0 & 3.396799 & -1.995769 & -2.042992 \\
\hline 35 & 1 & 0 & 3.385574 & -3.530893 & -1.137813 \\
\hline 36 & 1 & 0 & 4.679566 & -2.323704 & -0.830263 \\
\hline 37 & 8 & 0 & 1.875817 & 0.922066 & -0.811044 \\
\hline 38 & 8 & 0 & 2.344186 & 2.110117 & 1.054637 \\
\hline 39 & 6 & 0 & 3.152519 & 2.987666 & 0.253070 \\
\hline 40 & 1 & 0 & 3.561094 & 3.722446 & 0.947209 \\
\hline 41 & 1 & 0 & 3.955488 & 2.429476 & -0.235057 \\
\hline 42 & 1 & 0 & 2.544872 & 3.478145 & -0.512164 \\
\hline
\end{tabular}

\section{trans-3cf}

\begin{tabular}{|c|c|c|c|c|c|}
\hline \multirow{2}{*}{$\begin{array}{l}\text { Center } \\
\text { Number }\end{array}$} & Atomic & Atomic & \multicolumn{3}{|c|}{ Coordinates (Angstroms) } \\
\hline & Number & Type & $\mathrm{X}$ & $\mathrm{Y}$ & Z \\
\hline & & & 10 & --------1 & -------- \\
\hline 1 & 15 & 0 & 2.059729 & 0.434224 & 0.610247 \\
\hline 2 & 6 & 0 & 0.237474 & -0.088595 & 0.578627 \\
\hline 3 & 14 & 0 & -0.279966 & -0.654754 & 2.331899 \\
\hline 4 & 1 & 0 & -0.581899 & 0.544785 & 3.155622 \\
\hline 5 & 1 & 0 & 0.933559 & -1.287101 & 2.922607 \\
\hline 6 & 1 & 0 & -1.379645 & -1.637515 & 2.400021 \\
\hline 7 & 6 & 0 & -0.681220 & 0.851043 & -0.222426 \\
\hline 8 & 6 & 0 & -0.410046 & -0.559801 & -0.694849 \\
\hline 9 & 6 & 0 & -2.027422 & 1.187471 & 0.314433 \\
\hline 10 & 1 & 0 & -0.212960 & 1.650054 & -0.788427 \\
\hline 11 & 1 & 0 & 0.231287 & -0.628355 & -1.572737 \\
\hline 12 & 6 & 0 & -1.459944 & -1.641683 & -0.715235 \\
\hline 13 & 8 & 0 & -1.338448 & -2.726157 & -0.186204 \\
\hline 14 & 8 & 0 & -2.517597 & -1.289226 & -1.471184 \\
\hline 15 & 6 & 0 & -3.563139 & -2.273589 & -1.561969 \\
\hline 16 & 1 & 0 & -3.983178 & -2.468572 & -0.572123 \\
\hline 17 & 1 & 0 & -4.315839 & -1.839386 & -2.220494 \\
\hline 18 & 1 & 0 & -3.176754 & -3.207092 & -1.978987 \\
\hline 19 & 7 & 0 & 2.318370 & 1.498682 & -0.714373 \\
\hline 20 & 7 & 0 & 2.790219 & -1.014565 & -0.080591 \\
\hline 21 & 6 & 0 & 4.254075 & -0.934028 & -0.088493 \\
\hline 22 & 1 & 0 & 4.651669 & -1.690803 & -0.776364 \\
\hline 23 & 1 & 0 & 4.574481 & 0.048715 & -0.444786 \\
\hline 24 & 1 & 0 & 4.702907 & -1.107163 & 0.904852 \\
\hline 25 & 6 & 0 & 2.346001 & -2.336057 & 0.367668 \\
\hline 26 & 1 & 0 & 2.636691 & -2.565493 & 1.406845 \\
\hline 27 & 1 & 0 & 1.264621 & -2.445040 & 0.275915 \\
\hline 28 & 1 & 0 & 2.810864 & -3.092025 & -0.278286 \\
\hline 29 & 6 & 0 & 2.543038 & 2.909146 & -0.433663 \\
\hline 30 & 1 & 0 & 1.710477 & 3.541286 & -0.781604 \\
\hline 31 & 1 & 0 & 2.655034 & 3.058557 & 0.644259 \\
\hline 32 & 1 & 0 & 3.459313 & 3.263118 & -0.930700 \\
\hline 33 & 6 & 0 & 2.266538 & 1.158975 & -2.129615 \\
\hline 34 & 1 & 0 & 2.287112 & 0.072553 & -2.240955 \\
\hline 35 & 1 & 0 & 1.371631 & 1.562623 & -2.630608 \\
\hline 36 & 1 & 0 & 3.145878 & 1.570864 & -2.646560 \\
\hline 37 & 8 & 0 & -2.671857 & 0.488702 & 1.074928 \\
\hline 38 & 8 & 0 & -2.454042 & 2.379735 & -0.143965 \\
\hline 39 & 6 & 0 & -3.749993 & 2.791634 & 0.324255 \\
\hline 40 & 1 & 0 & -3.925878 & 3.768129 & -0.127479 \\
\hline 41 & 1 & 0 & -3.757692 & 2.863309 & 1.414874 \\
\hline 42 & 1 & 0 & -4.514809 & 2.077401 & 0.008584 \\
\hline
\end{tabular}

cis $-3 d b$ 


\begin{tabular}{|c|c|c|c|c|c|}
\hline Center & Atomic & Atomic & \multicolumn{3}{|c|}{ Coordinates (Angstroms) } \\
\hline Number & Number & Type & $\mathrm{X}$ & $\mathrm{Y}$ & $\mathrm{Z}$ \\
\hline & & & & & ---------- \\
\hline 1 & 6 & 0 & -1.298855 & 1.135630 & -0.771440 \\
\hline 2 & 6 & 0 & -0.357290 & 1.092219 & -1.932544 \\
\hline 3 & 6 & 0 & 0.155043 & 0.608833 & -0.576809 \\
\hline 4 & 6 & 0 & -2.507372 & 0.262105 & -0.721862 \\
\hline 5 & 8 & 0 & -3.398442 & 0.762326 & 0.172587 \\
\hline 6 & 8 & 0 & -2.714312 & -0.744279 & -1.368358 \\
\hline 7 & 15 & 0 & 0.317689 & -1.283764 & -0.594848 \\
\hline 8 & 7 & 0 & 2.094598 & -1.393506 & -0.551257 \\
\hline 9 & 7 & 0 & -0.057846 & -1.860073 & 0.969223 \\
\hline 10 & 14 & 0 & 1.138793 & 1.873663 & 0.491356 \\
\hline 11 & 6 & 0 & 0.299521 & 2.072997 & 2.181007 \\
\hline 12 & 6 & 0 & 2.974118 & 1.493731 & 0.754390 \\
\hline 13 & 6 & 0 & 1.064882 & 3.568203 & -0.373123 \\
\hline 14 & 6 & 0 & 0.625342 & -1.546420 & 2.211524 \\
\hline 15 & 6 & 0 & -1.199872 & -2.749454 & 1.124726 \\
\hline 16 & 6 & 0 & 2.716057 & -0.840950 & -1.758131 \\
\hline 17 & 6 & 0 & 2.552839 & -2.772108 & -0.354111 \\
\hline 18 & 6 & 0 & -4.616636 & 0.015251 & 0.305420 \\
\hline 19 & 1 & 0 & -1.492929 & 2.106837 & -0.324823 \\
\hline 20 & 1 & 0 & -0.012164 & 2.039136 & -2.339072 \\
\hline 21 & 1 & 0 & -0.564561 & 0.327953 & -2.679281 \\
\hline 22 & 1 & 0 & 0.711502 & 2.948560 & 2.698511 \\
\hline 23 & 1 & 0 & 0.452527 & 1.206559 & 2.831275 \\
\hline 24 & 1 & 0 & -0.781496 & 2.224938 & 2.082617 \\
\hline 25 & 1 & 0 & 3.394633 & 2.233286 & 1.448257 \\
\hline 26 & 1 & 0 & 3.539222 & 1.564014 & -0.181593 \\
\hline 27 & 1 & 0 & 3.145255 & 0.494968 & 1.162674 \\
\hline 28 & 1 & 0 & 1.604469 & 3.565508 & -1.327809 \\
\hline 29 & 1 & 0 & 1.548369 & 4.316026 & 0.268195 \\
\hline 30 & 1 & 0 & 0.045445 & 3.921954 & -0.567024 \\
\hline 31 & 1 & 0 & 0.943671 & -2.469963 & 2.721026 \\
\hline 32 & 1 & 0 & -0.025382 & -0.995388 & 2.908181 \\
\hline 33 & 1 & 0 & 1.518081 & -0.952325 & 2.010717 \\
\hline 34 & 1 & 0 & -0.895676 & -3.707892 & 1.575430 \\
\hline 35 & 1 & 0 & -1.654691 & -2.947504 & 0.151603 \\
\hline 36 & 1 & 0 & -1.968227 & -2.305493 & 1.778131 \\
\hline 37 & 1 & 0 & 3.806018 & -0.840732 & -1.634243 \\
\hline 38 & 1 & 0 & 2.396883 & 0.190822 & -1.918267 \\
\hline 39 & 1 & 0 & 2.480437 & -1.417966 & -2.671369 \\
\hline 40 & 1 & 0 & 3.645047 & -2.770150 & -0.249960 \\
\hline 41 & 1 & 0 & 2.294701 & -3.443274 & -1.193948 \\
\hline 42 & 1 & 0 & 2.124094 & -3.189929 & 0.559132 \\
\hline 43 & 1 & 0 & -5.221341 & 0.566200 & 1.026753 \\
\hline 44 & 1 & 0 & -4.411027 & -0.994993 & 0.670350 \\
\hline 45 & 1 & 0 & -5.133213 & -0.055527 & -0.655586 \\
\hline
\end{tabular}

\section{trans $-3 d b$}

\begin{tabular}{|c|c|c|c|c|c|}
\hline Center & Atomic & Atomic & \multicolumn{3}{|c|}{ Coordinates (Angstroms) } \\
\hline Number & Number & Type & $\mathrm{X}$ & $\mathrm{Y}$ & Z \\
\hline & & & --------- & --------- & --------- \\
\hline 1 & 6 & 0 & -1.259310 & -0.570543 & -1.133909 \\
\hline 2 & 6 & 0 & -0.649016 & 0.476945 & -2.012677 \\
\hline 3 & 6 & 0 & -0.056281 & 0.279179 & -0.621277 \\
\hline 4 & 15 & 0 & 1.415556 & -0.920972 & -0.745831 \\
\hline 5 & 7 & 0 & 2.782005 & 0.164316 & -0.472410 \\
\hline 6 & 7 & 0 & 1.484672 & -1.854637 & 0.683626 \\
\hline 7 & 14 & 0 & -0.315140 & 1.712545 & 0.656078 \\
\hline 8 & 6 & 0 & -1.274276 & 1.121973 & 2.182448 \\
\hline
\end{tabular}




\begin{tabular}{|c|c|c|c|c|c|}
\hline 9 & 6 & 0 & 1.312035 & 2.479027 & 1.262661 \\
\hline 10 & 6 & 0 & -1.255449 & 3.147933 & -0.150078 \\
\hline 11 & 6 & 0 & -2.649020 & -0.419661 & -0.626710 \\
\hline 12 & 6 & 0 & 1.619468 & -1.351263 & 2.041175 \\
\hline 13 & 6 & 0 & 1.073557 & -3.250079 & 0.610801 \\
\hline 14 & 6 & 0 & 2.903270 & 1.189021 & -1.512165 \\
\hline 15 & 6 & 0 & 4.057679 & -0.539126 & -0.313500 \\
\hline 16 & 8 & 0 & -3.270886 & 0.616633 & -0.488333 \\
\hline 17 & 8 & 0 & -3.177284 & -1.634061 & -0.341148 \\
\hline 18 & 6 & 0 & -4.531078 & -1.618820 & 0.137120 \\
\hline 19 & 1 & 0 & -1.021926 & -1.604279 & -1.375866 \\
\hline 20 & 1 & 0 & -1.238367 & 1.371715 & -2.188479 \\
\hline 21 & 1 & 0 & -0.094880 & 0.120229 & -2.879718 \\
\hline 22 & 1 & 0 & -1.319957 & 1.934592 & 2.918762 \\
\hline 23 & 1 & 0 & -0.802379 & 0.264157 & 2.673127 \\
\hline 24 & 1 & 0 & -2.302885 & 0.850717 & 1.927600 \\
\hline 25 & 1 & 0 & 1.058939 & 3.240083 & 2.013202 \\
\hline 26 & 1 & 0 & 1.847638 & 2.987521 & 0.454620 \\
\hline 27 & 1 & 0 & 2.007830 & 1.770721 & 1.716726 \\
\hline 28 & 1 & 0 & -0.706653 & 3.564873 & -1.003774 \\
\hline 29 & 1 & 0 & -1.372086 & 3.955476 & 0.584333 \\
\hline 30 & 1 & 0 & -2.250444 & 2.847236 & -0.486781 \\
\hline 31 & 1 & 0 & 2.344487 & -1.962110 & 2.599771 \\
\hline 32 & 1 & 0 & 0.666367 & -1.384628 & 2.591792 \\
\hline 33 & 1 & 0 & 1.984863 & -0.324337 & 2.023958 \\
\hline 34 & 1 & 0 & 1.775206 & -3.883448 & 1.173702 \\
\hline 35 & 1 & 0 & 1.069599 & -3.583827 & -0.430467 \\
\hline 36 & 1 & 0 & 0.066820 & -3.411437 & 1.029479 \\
\hline 37 & 1 & 0 & 3.665734 & 1.918114 & -1.211850 \\
\hline 38 & 1 & 0 & 1.959868 & 1.723873 & -1.639512 \\
\hline 39 & 1 & 0 & 3.199546 & 0.776824 & -2.494250 \\
\hline 40 & 1 & 0 & 4.829186 & 0.188459 & -0.032579 \\
\hline 41 & 1 & 0 & 4.393312 & -1.047925 & -1.235919 \\
\hline 42 & 1 & 0 & 3.983268 & -1.285644 & 0.479442 \\
\hline 43 & 1 & 0 & -4.802247 & -2.665365 & 0.280101 \\
\hline 44 & 1 & 0 & -5.192874 & -1.145699 & -0.593157 \\
\hline 45 & 1 & 0 & -4.600189 & -1.072799 & 1.082121 \\
\hline
\end{tabular}

cis-3dc

\begin{tabular}{|c|c|c|c|c|c|}
\hline Center & Atomic & Atomic & \multicolumn{3}{|c|}{ Coordinates (Angstroms) } \\
\hline Number & Number & Type & $\mathrm{X}$ & $\mathrm{Y}$ & $\mathrm{Z}$ \\
\hline------ & & & --------- & --------- & ------------ \\
\hline 1 & 6 & 0 & -1.116707 & 1.205227 & -0.876860 \\
\hline 2 & 6 & 0 & -0.107607 & 1.107747 & -1.960839 \\
\hline 3 & 6 & 0 & 0.293437 & 0.624903 & -0.557363 \\
\hline 4 & 6 & 0 & -2.381572 & 0.369626 & -1.025858 \\
\hline 5 & 7 & 0 & -3.191199 & 0.267086 & 0.083178 \\
\hline 6 & 8 & 0 & -2.690582 & -0.130824 & -2.102957 \\
\hline 7 & 15 & 0 & 0.259739 & -1.253909 & -0.459917 \\
\hline 8 & 7 & 0 & 1.981050 & -1.622177 & -0.715159 \\
\hline 9 & 7 & 0 & 0.100839 & -1.679363 & 1.197592 \\
\hline 10 & 14 & 0 & 1.412117 & 1.823725 & 0.435086 \\
\hline 11 & 6 & 0 & 0.820696 & 2.029996 & 2.227524 \\
\hline 12 & 6 & 0 & 3.240536 & 1.325297 & 0.419014 \\
\hline 13 & 6 & 0 & 1.319898 & 3.559956 & -0.339308 \\
\hline 14 & 6 & 0 & 1.114272 & -1.555353 & 2.232788 \\
\hline 15 & 6 & 0 & -0.996699 & -2.562357 & 1.568161 \\
\hline 16 & 6 & 0 & 2.529162 & -1.124653 & -1.979359 \\
\hline 17 & 6 & 0 & 2.238853 & -3.061043 & -0.598502 \\
\hline 18 & 6 & 0 & -4.497693 & -0.358264 & -0.049940 \\
\hline 19 & 6 & 0 & -2.870594 & 0.810015 & 1.390165 \\
\hline 20 & 1 & 0 & -1.309334 & 2.192711 & -0.459462 \\
\hline
\end{tabular}




\begin{tabular}{|c|c|c|c|c|c|}
\hline 21 & 1 & 0 & 0.339589 & 2.018041 & -2.349423 \\
\hline 22 & 1 & 0 & -0.321438 & 0.356053 & -2.717249 \\
\hline 23 & 1 & 0 & 1.500421 & 2.709273 & 2.757821 \\
\hline 24 & 1 & 0 & 0.788829 & 1.092283 & 2.788413 \\
\hline 25 & 1 & 0 & -0.178699 & 2.479434 & 2.270265 \\
\hline 26 & 1 & 0 & 3.801104 & 1.943455 & 1.131741 \\
\hline 27 & 1 & 0 & 3.679819 & 1.490660 & -0.571826 \\
\hline 28 & 1 & 0 & 3.396866 & 0.272579 & 0.669128 \\
\hline 29 & 1 & 0 & 1.760885 & 3.595927 & -1.342067 \\
\hline 30 & 1 & 0 & 1.891500 & 4.255547 & 0.288544 \\
\hline 31 & 1 & 0 & 0.299534 & 3.954882 & -0.409159 \\
\hline 32 & 1 & 0 & 1.508840 & -2.542920 & 2.525207 \\
\hline 33 & 1 & 0 & 0.699173 & -1.091089 & 3.139896 \\
\hline 34 & 1 & 0 & 1.953336 & -0.957121 & 1.878453 \\
\hline 35 & 1 & 0 & -0.635704 & -3.566969 & 1.847241 \\
\hline 36 & 1 & 0 & -1.691861 & -2.667019 & 0.731606 \\
\hline 37 & 1 & 0 & -1.550180 & -2.160013 & 2.430359 \\
\hline 38 & 1 & 0 & 3.606467 & -1.330773 & -2.005236 \\
\hline 39 & 1 & 0 & 2.391393 & -0.045888 & -2.060030 \\
\hline 40 & 1 & 0 & 2.075596 & -1.598749 & -2.869005 \\
\hline 41 & 1 & 0 & 3.322468 & -3.231949 & -0.614830 \\
\hline 42 & 1 & 0 & 1.788991 & -3.652753 & -1.416050 \\
\hline 43 & 1 & 0 & 1.852127 & -3.442352 & 0.349639 \\
\hline 44 & 1 & 0 & -4.586855 & -1.214491 & 0.631759 \\
\hline 45 & 1 & 0 & -4.610613 & -0.698958 & -1.078162 \\
\hline 46 & 1 & 0 & -5.296366 & 0.357005 & 0.191235 \\
\hline 47 & 1 & 0 & -1.798455 & 0.973067 & 1.484405 \\
\hline 48 & 1 & 0 & -3.400466 & 1.755023 & 1.586368 \\
\hline 49 & 1 & 0 & -3.168755 & 0.092782 & 2.164619 \\
\hline
\end{tabular}

\section{trans-3dc}

\begin{tabular}{|c|c|c|c|c|c|}
\hline \multirow{2}{*}{$\begin{array}{l}\text { Center } \\
\text { Number }\end{array}$} & \multirow{2}{*}{$\begin{array}{l}\text { Atomic } \\
\text { Number }\end{array}$} & Atomic & \multicolumn{3}{|c|}{ Coordinates (Angstroms) } \\
\hline & & Type & $\mathrm{X}$ & $\mathrm{Y}$ & $\mathrm{Z}$ \\
\hline 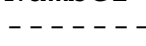 & & & -------1 & --------- & -------- \\
\hline 1 & 6 & 0 & -1.132350 & -0.274918 & -1.298129 \\
\hline 2 & 6 & 0 & -0.437260 & 0.819800 & -2.027629 \\
\hline 3 & 6 & 0 & 0.086346 & 0.436750 & -0.637263 \\
\hline 4 & 15 & 0 & 1.518864 & -0.770875 & -0.825373 \\
\hline 5 & 7 & 0 & 2.905049 & 0.246739 & -0.397557 \\
\hline 6 & 7 & 0 & 1.430385 & -1.839349 & 0.515977 \\
\hline 7 & 14 & 0 & -0.207530 & 1.712298 & 0.786807 \\
\hline 8 & 6 & 0 & -1.252355 & 0.934828 & 2.168886 \\
\hline 9 & 6 & 0 & 1.398371 & 2.384562 & 1.546154 \\
\hline 10 & 6 & 0 & -1.118381 & 3.236403 & 0.127398 \\
\hline 11 & 6 & 0 & -2.551933 & -0.083298 & -0.824975 \\
\hline 12 & 6 & 0 & 1.608780 & -1.480649 & 1.916047 \\
\hline 13 & 6 & 0 & 1.604783 & -3.265490 & 0.265295 \\
\hline 14 & 6 & 0 & 3.093604 & 1.377259 & -1.308919 \\
\hline 15 & 6 & 0 & 4.146318 & -0.519962 & -0.278492 \\
\hline 16 & 8 & 0 & -3.167299 & 0.965181 & -1.023185 \\
\hline 17 & 7 & 0 & -3.140961 & -1.154944 & -0.196317 \\
\hline 18 & 6 & 0 & -4.551162 & -1.080221 & 0.154938 \\
\hline 19 & 6 & 0 & -2.442164 & -2.353972 & 0.239855 \\
\hline 20 & 1 & 0 & -0.918526 & -1.279942 & -1.658694 \\
\hline 21 & 1 & 0 & -0.974365 & 1.757250 & -2.126219 \\
\hline 22 & 1 & 0 & 0.148984 & 0.536711 & -2.900257 \\
\hline 23 & 1 & 0 & -1.306376 & 1.622588 & 3.022241 \\
\hline 24 & 1 & 0 & -0.841243 & -0.012210 & 2.533961 \\
\hline 25 & 1 & 0 & -2.277128 & 0.750603 & 1.830501 \\
\hline 26 & 1 & 0 & 1.128271 & 3. 014822 & 2.404088 \\
\hline 27 & 1 & 0 & 1.932180 & 3.022024 & 0.832453 \\
\hline 28 & 1 & 0 & 2.104827 & 1.624460 & 1.885656 \\
\hline
\end{tabular}




\begin{tabular}{|c|c|c|c|c|c|}
\hline 29 & 1 & 0 & -0.524042 & 3.765825 & -0.627683 \\
\hline 30 & 1 & 0 & -1.292288 & 3.937782 & 0.953878 \\
\hline 31 & 1 & 0 & -2.083658 & 2.973488 & -0.312992 \\
\hline 32 & 1 & 0 & 2.594887 & -1.797919 & 2.293880 \\
\hline 33 & 1 & 0 & 0.844824 & -1.967977 & 2.539579 \\
\hline 34 & 1 & 0 & 1.532184 & -0.403596 & 2.050256 \\
\hline 35 & 1 & 0 & 2.599566 & -3.622273 & 0.582235 \\
\hline 36 & 1 & 0 & 1.490165 & -3.475801 & -0.801062 \\
\hline 37 & 1 & 0 & 0.853670 & -3.850184 & 0.816842 \\
\hline 38 & 1 & 0 & 3.882076 & 2.030309 & -0.914707 \\
\hline 39 & 1 & 0 & 2.176820 & 1.965849 & -1.383445 \\
\hline 40 & 1 & 0 & 3.389388 & 1.070719 & -2.329331 \\
\hline 41 & 1 & 0 & 4.935978 & 0.135938 & 0.108770 \\
\hline 42 & 1 & 0 & 4.498323 & -0.940739 & -1.238568 \\
\hline 43 & 1 & 0 & 4.018825 & -1.344048 & 0.428262 \\
\hline 44 & 1 & 0 & -5.078433 & -1.970229 & -0.211847 \\
\hline 45 & 1 & 0 & -4.975235 & -0.185752 & -0.298930 \\
\hline 46 & 1 & 0 & -4.681915 & -1.026939 & 1.244851 \\
\hline 47 & 1 & 0 & -2.738617 & -2.588799 & 1.270076 \\
\hline 48 & 1 & 0 & -2.692655 & -3.221453 & -0.388164 \\
\hline 49 & 1 & 0 & -1.362533 & -2.204682 & 0.231428 \\
\hline
\end{tabular}

cis-3dd

\begin{tabular}{|c|c|c|c|c|c|}
\hline Center & Atomic & Atomic & \multicolumn{3}{|c|}{ Coordinates (Angstroms) } \\
\hline Number & Number & Type & $\mathrm{X}$ & $\mathrm{Y}$ & $\mathrm{Z}$ \\
\hline & & & & & \\
\hline 1 & 6 & 0 & 0.399082 & -1.476236 & -0.585536 \\
\hline 2 & 6 & 0 & -0.160687 & -1.001756 & -1.888049 \\
\hline 3 & 6 & 0 & 1.817003 & -1.261625 & -0.148935 \\
\hline 4 & 6 & 0 & 2.180144 & -1.540769 & 1.179358 \\
\hline 5 & 6 & 0 & 2.831751 & -0.897545 & -1.044581 \\
\hline 6 & 6 & 0 & 4.157212 & -0.783978 & -0.621683 \\
\hline 7 & 6 & 0 & 3.502576 & -1.425376 & 1.607228 \\
\hline 8 & 6 & 0 & 4.498632 & -1.041128 & 0.706801 \\
\hline 9 & 1 & 0 & 0.067026 & -2.475847 & -0.297379 \\
\hline 10 & 1 & 0 & -0.698967 & -1.702581 & -2.522055 \\
\hline 11 & 1 & 0 & 0.434094 & -0.276696 & -2.439535 \\
\hline 12 & 1 & 0 & 1.414917 & -1.863140 & 1.882493 \\
\hline 13 & 1 & 0 & 2.583094 & -0.706456 & -2.083598 \\
\hline 14 & 1 & 0 & 4.926195 & -0.500788 & -1.336151 \\
\hline 15 & 1 & 0 & 3.756126 & -1.643907 & 2.641508 \\
\hline 16 & 1 & 0 & 5.530794 & -0.953744 & 1.035359 \\
\hline 17 & 6 & 0 & -0.794086 & -0.474280 & -0.600783 \\
\hline 18 & 15 & 0 & -0.856750 & 1.387750 & -0.509442 \\
\hline 19 & 7 & 0 & 0.722184 & 1.971429 & -0.961219 \\
\hline 20 & 7 & 0 & -1.123477 & 1.654613 & 1.179458 \\
\hline 21 & 14 & 0 & -2.471146 & -1.261500 & -0.102941 \\
\hline 22 & 6 & 0 & -2.507668 & -1.692704 & 1.742750 \\
\hline 23 & 6 & 0 & -3.937230 & -0.136170 & -0.516056 \\
\hline 24 & 6 & 0 & -2.712604 & -2.883680 & -1.065599 \\
\hline 25 & 6 & 0 & -1.746279 & 2.926136 & 1.534564 \\
\hline 26 & 6 & 0 & -0.337346 & 1.096018 & 2.272155 \\
\hline 27 & 6 & 0 & 0.722786 & 3.022695 & -1.977867 \\
\hline 28 & 6 & 0 & 1.832198 & 2.126788 & -0.027623 \\
\hline 29 & 1 & 0 & -3.416111 & -2.260573 & 1.979985 \\
\hline 30 & 1 & 0 & -2.504024 & -0.794356 & 2.367351 \\
\hline 31 & 1 & 0 & -1.649974 & -2.310422 & 2.035732 \\
\hline 32 & 1 & 0 & -4.879505 & -0.659843 & -0.309170 \\
\hline 33 & 1 & 0 & -3.939406 & 0.151939 & -1.573284 \\
\hline 34 & 1 & 0 & -3.925589 & 0.783690 & 0.076952 \\
\hline 35 & 1 & 0 & -2.851992 & -2.708368 & -2.138571 \\
\hline 36 & 1 & 0 & -3.616224 & -3.387846 & -0.700193 \\
\hline
\end{tabular}




\begin{tabular}{|c|c|c|c|c|c|}
\hline 37 & 1 & 0 & -1.881076 & -3.588186 & -0.946392 \\
\hline 38 & 1 & 0 & -1.008568 & 3.711854 & 1.772496 \\
\hline 39 & 1 & 0 & -2.386392 & 2.796569 & 2.418956 \\
\hline 40 & 1 & 0 & -2.369032 & 3.279461 & 0.707840 \\
\hline 41 & 1 & 0 & 0.475241 & 1.766499 & 2.596644 \\
\hline 42 & 1 & 0 & 0.101172 & 0.143262 & 1.974644 \\
\hline 43 & 1 & 0 & -0.984135 & 0.918030 & 3.143509 \\
\hline 44 & 1 & 0 & 0.708346 & 4.037323 & -1.541219 \\
\hline 45 & 1 & 0 & -0.149661 & 2.920142 & -2.627841 \\
\hline 46 & 1 & 0 & 1.626156 & 2.943401 & -2.599234 \\
\hline 47 & 1 & 0 & 1.762039 & 3.069151 & 0.543450 \\
\hline 48 & 1 & 0 & 2.782816 & 2.140638 & -0.577024 \\
\hline 49 & 1 & 0 & 1.876083 & 1.294905 & 0.672999 \\
\hline
\end{tabular}

\section{trans-3dd}

\begin{tabular}{|c|c|c|c|c|c|}
\hline \multirow{2}{*}{$\begin{array}{l}\text { Center } \\
\text { Number }\end{array}$} & Atomic & Atomic & \multicolumn{3}{|c|}{ Coordinates (Angstroms) } \\
\hline & Number & Type & $\mathrm{X}$ & $\mathrm{Y}$ & Z \\
\hline & ---1 & & -------1 & -------- & ------- \\
\hline 1 & 6 & 0 & 0.707010 & -0.519103 & 2.463825 \\
\hline 2 & 14 & 0 & 0.087581 & -1.472275 & 0.946491 \\
\hline 3 & 6 & 0 & 1.410372 & -2.772726 & 0.529444 \\
\hline 4 & 6 & 0 & -0.243647 & -0.321716 & -0.556778 \\
\hline 5 & 15 & 0 & -1.773020 & 0.746846 & -0.859756 \\
\hline 6 & 7 & 0 & -1.995988 & 1.799204 & 0.469672 \\
\hline 7 & 6 & 0 & -1.852883 & 3.231598 & 0.259705 \\
\hline 8 & 6 & 0 & 0.932916 & 0.422927 & -1.251482 \\
\hline 9 & 6 & 0 & 2.346028 & 0.497014 & -0.753852 \\
\hline 10 & 6 & 0 & 0.329826 & -0.764520 & -1.916398 \\
\hline 11 & 7 & 0 & -3.090936 & -0.399665 & -0.559562 \\
\hline 12 & 6 & 0 & -4.410595 & 0.235296 & -0.612864 \\
\hline 13 & 6 & 0 & -3.066757 & -1.568552 & -1.441243 \\
\hline 14 & 6 & 0 & -2.335853 & 1.433859 & 1.834504 \\
\hline 15 & 6 & 0 & -1.446674 & -2.468765 & 1.452066 \\
\hline 16 & 1 & 0 & 0.618221 & 1.373340 & -1.689946 \\
\hline 17 & 1 & 0 & 0.890632 & -1.694571 & -1.940437 \\
\hline 18 & 1 & 0 & -0.264271 & -0.593294 & -2.813239 \\
\hline 19 & 1 & 0 & 0.857327 & -1.220648 & 3.294548 \\
\hline 20 & 1 & 0 & 0.003424 & 0.245901 & 2.804762 \\
\hline 21 & 1 & 0 & 1.665324 & -0.030187 & 2.263524 \\
\hline 22 & 1 & 0 & -1.238455 & -2.982283 & 2.399706 \\
\hline 23 & 1 & 0 & -1.684435 & -3.240749 & 0.710823 \\
\hline 24 & 1 & 0 & -2.342273 & -1.855450 & 1.577533 \\
\hline 25 & 1 & 0 & 1.117131 & -3.409755 & -0.313804 \\
\hline 26 & 1 & 0 & 1.533517 & -3.430614 & 1.399817 \\
\hline 27 & 1 & 0 & 2.388233 & -2.336945 & 0.304059 \\
\hline 28 & 1 & 0 & -3.271335 & 1.926351 & 2.145396 \\
\hline 29 & 1 & 0 & -1.552889 & 1.741442 & 2.544122 \\
\hline 30 & 1 & 0 & -2.482745 & 0.356232 & 1.911164 \\
\hline 31 & 1 & 0 & -2.763943 & 3.767940 & 0.570430 \\
\hline 32 & 1 & 0 & -1.677480 & 3.438356 & -0.799409 \\
\hline 33 & 1 & 0 & -1.011707 & 3.647201 & 0.837277 \\
\hline 34 & 1 & 0 & -3.830250 & -2.283502 & -1.110443 \\
\hline 35 & 1 & 0 & -2.097267 & -2.066156 & -1.393106 \\
\hline 36 & 1 & 0 & -3.275558 & -1.319747 & -2.498346 \\
\hline 37 & 1 & 0 & -5.167381 & -0.489488 & -0.287716 \\
\hline 38 & 1 & 0 & -4.687680 & 0.581589 & -1.625518 \\
\hline 39 & 1 & 0 & -4.447960 & 1.093001 & 0.062078 \\
\hline 40 & 6 & 0 & 3.365498 & -0.306361 & -1.281829 \\
\hline 41 & 6 & 0 & 4.687635 & -0.165062 & -0.853194 \\
\hline 42 & 6 & 0 & 5.016533 & 0.793066 & 0.105566 \\
\hline 43 & 6 & 0 & 4.013696 & 1.613753 & 0.628180 \\
\hline 44 & 6 & 0 & 2.695295 & 1.467387 & 0.199283 \\
\hline
\end{tabular}




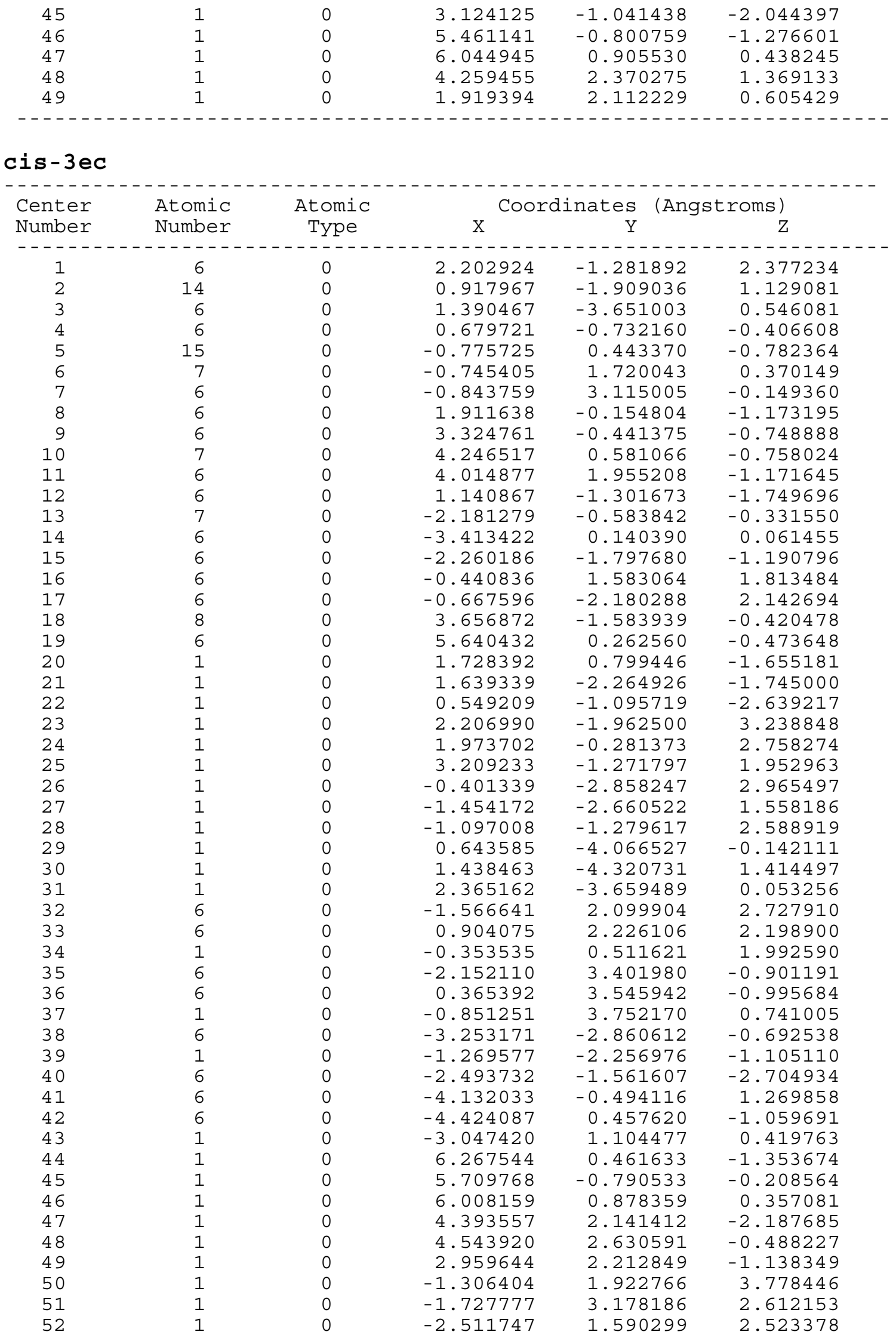

S.53 


\begin{tabular}{|c|c|c|c|c|c|}
\hline 53 & 1 & 0 & 1.138492 & 2.013191 & 3.248640 \\
\hline 54 & 1 & 0 & 1.717887 & 1.833236 & 1.582050 \\
\hline 55 & 1 & 0 & 0.881004 & 3.316541 & 2.088239 \\
\hline 56 & 1 & 0 & -2.184394 & 4.454095 & -1.209241 \\
\hline 57 & 1 & 0 & -2.233871 & 2.786509 & -1.802955 \\
\hline 58 & 1 & 0 & -3.024931 & 3.207734 & -0.270636 \\
\hline 59 & 1 & 0 & 0.278253 & 4.601610 & -1.281420 \\
\hline 60 & 1 & 0 & 1.296843 & 3.424824 & -0.435028 \\
\hline 61 & 1 & 0 & 0.427292 & 2.951996 & -1.913827 \\
\hline 62 & 1 & 0 & -5.188059 & 1.147708 & -0.679475 \\
\hline 63 & 1 & 0 & -3.938139 & 0.933835 & -1.916614 \\
\hline 64 & 1 & 0 & -4.947231 & -0.435814 & -1.416007 \\
\hline 65 & 1 & 0 & -4.864828 & 0.215976 & 1.675173 \\
\hline 66 & 1 & 0 & -4.675472 & -1.406347 & 1.010886 \\
\hline 67 & 1 & 0 & -3.417501 & -0.739144 & 2.060535 \\
\hline 68 & 1 & 0 & -3.094769 & -3.784658 & -1.261156 \\
\hline 69 & 1 & 0 & -3.116796 & -3.088489 & 0.367431 \\
\hline 70 & 1 & 0 & -4.294524 & -2.562047 & -0.848975 \\
\hline 71 & 1 & 0 & -2.266760 & -2.481472 & -3.258440 \\
\hline 72 & 1 & 0 & -3.531260 & -1.294330 & -2.924146 \\
\hline $7 \overline{3}$ & 1 & 0 & -1.852633 & -0.766623 & -3.097115 \\
\hline
\end{tabular}

\section{trans-3ec}

\begin{tabular}{|c|c|c|c|c|c|}
\hline Center & Atomic & Atomic & \multicolumn{3}{|c|}{ Coordinates (Angstroms) } \\
\hline Number & Number & Type & $\mathrm{X}$ & $\mathrm{Y}$ & $\mathrm{Z}$ \\
\hline & & & & & \\
\hline 1 & 6 & 0 & -1.896333 & 0.059261 & -1.279836 \\
\hline 2 & 6 & 0 & -0.892726 & 0.797678 & -2.119485 \\
\hline 3 & 6 & 0 & -1.908148 & -1.438935 & -1.334357 \\
\hline 4 & 7 & 0 & -3.076323 & -2.073244 & -0.969342 \\
\hline 5 & 8 & 0 & -0.932189 & -2.086960 & -1.722457 \\
\hline 6 & 6 & 0 & -3.192945 & -3.508538 & -1.195403 \\
\hline 7 & 6 & 0 & -4.285303 & -1.404950 & -0.507057 \\
\hline 8 & 1 & 0 & -2.883922 & 0.504059 & -1.261356 \\
\hline 9 & 1 & 0 & -1.253039 & 1.608841 & -2.751973 \\
\hline 10 & 1 & 0 & -0.125513 & 0.180060 & -2.575391 \\
\hline 11 & 1 & 0 & -3.456101 & -4.023415 & -0.262677 \\
\hline 12 & 1 & 0 & -2.235089 & -3.877522 & -1.557607 \\
\hline 13 & 1 & 0 & -3.972182 & -3.722806 & -1.939722 \\
\hline 14 & 1 & 0 & -4.050185 & -0.545290 & 0.121504 \\
\hline 15 & 1 & 0 & -4.928104 & -1.077261 & -1.337394 \\
\hline 16 & 1 & 0 & -4.858816 & -2.108187 & 0.104903 \\
\hline 17 & 6 & 0 & -0.808692 & 0.973963 & -0.620925 \\
\hline 18 & 15 & 0 & 0.860974 & 0.760891 & 0.261200 \\
\hline 19 & 7 & 0 & 2.012706 & -0.000897 & -0.794230 \\
\hline 20 & 7 & 0 & 0.539235 & -0.197418 & 1.664020 \\
\hline 21 & 14 & 0 & -1.474857 & 2.682252 & -0.031606 \\
\hline 22 & 6 & 0 & -1.589957 & 2.830211 & 1.852786 \\
\hline 23 & 6 & 0 & -0.353215 & 4.055730 & -0.698388 \\
\hline 24 & 6 & 0 & -3.222329 & 2.986662 & -0.723878 \\
\hline 25 & 6 & 0 & 1.265100 & 0.288543 & 2.872618 \\
\hline 26 & 6 & 0 & -0.289359 & -1.427794 & 1.679820 \\
\hline 27 & 6 & 0 & 3.035967 & 0.958206 & -1.294154 \\
\hline 28 & 6 & 0 & 2.045347 & -1.445912 & -1.134243 \\
\hline 29 & 6 & 0 & 2.630918 & -0.384458 & 3.112471 \\
\hline 30 & 6 & 0 & 0.434177 & 0.304587 & 4.166770 \\
\hline 31 & 6 & 0 & 0.366759 & -2.661610 & 2.326397 \\
\hline 32 & 6 & 0 & -1.700612 & -1.210113 & 2.264108 \\
\hline 33 & 6 & 0 & 4.494513 & 0.496714 & -1.135083 \\
\hline 34 & 6 & 0 & 2.781014 & 1.471864 & -2.726105 \\
\hline 35 & 6 & 0 & 3.078036 & -2.262384 & -0.327693 \\
\hline 36 & 6 & 0 & 2.191398 & -1.762656 & -2.632195 \\
\hline
\end{tabular}




\begin{tabular}{|c|c|c|c|c|c|}
\hline 37 & 1 & 0 & -1.997507 & 3.812733 & 2.123229 \\
\hline 38 & 1 & 0 & -0.608583 & 2.734455 & 2.327008 \\
\hline 39 & 1 & 0 & -2.247658 & 2.068176 & 2.285715 \\
\hline 40 & 1 & 0 & 0.653538 & 3.982270 & -0.275662 \\
\hline 41 & 1 & 0 & -0.758819 & 5.042669 & -0.442090 \\
\hline 42 & 1 & 0 & -0.261876 & 4.009038 & -1.790121 \\
\hline 43 & 1 & 0 & -3.281153 & 2.875128 & -1.813198 \\
\hline 44 & 1 & 0 & -3.521392 & 4.016546 & -0.491083 \\
\hline 45 & 1 & 0 & -3.975084 & 2.326251 & -0.276486 \\
\hline 46 & 1 & 0 & 1.487301 & 1.338007 & 2.645474 \\
\hline 47 & 1 & 0 & -0.429185 & -1.688255 & 0.631631 \\
\hline 48 & 1 & 0 & 2.924685 & 1.831763 & -0.640913 \\
\hline 49 & 1 & 0 & 1.060223 & -1.820529 & -0.861849 \\
\hline 50 & 1 & 0 & 2.536127 & -1.437396 & 3.393534 \\
\hline 51 & 1 & 0 & 3.161992 & 0.129391 & 3.924044 \\
\hline 52 & 1 & 0 & 3.251576 & -0.323803 & 2.213727 \\
\hline 53 & 1 & 0 & 1.005774 & 0.822252 & 4.946404 \\
\hline 54 & 1 & 0 & 0.215348 & -0.699357 & 4.543763 \\
\hline 55 & 1 & 0 & -0.510244 & 0.838764 & 4.031445 \\
\hline 56 & 1 & 0 & 1.341367 & -2.879596 & 1.883284 \\
\hline 57 & 1 & 0 & -0.277784 & -3.534028 & 2.163914 \\
\hline 58 & 1 & 0 & 0.494844 & -2.551290 & 3.407749 \\
\hline 59 & 1 & 0 & -2.328788 & -2.083569 & 2.049551 \\
\hline 60 & 1 & 0 & -2.169517 & -0.331005 & 1.813308 \\
\hline 61 & 1 & 0 & -1.688368 & -1.071109 & 3.348255 \\
\hline 62 & 1 & 0 & 5.162172 & 1.334483 & -1.370507 \\
\hline 63 & 1 & 0 & 4.754576 & -0.323204 & -1.811852 \\
\hline 64 & 1 & 0 & 4.702447 & 0.177701 & -0.109189 \\
\hline 65 & 1 & 0 & 3.487143 & 2.278573 & -2.961907 \\
\hline 66 & 1 & 0 & 1.767742 & 1.875637 & -2.818204 \\
\hline 67 & 1 & 0 & 2.906107 & 0.691546 & -3.481933 \\
\hline 68 & 1 & 0 & 3.044876 & -2.000765 & 0.732173 \\
\hline 69 & 1 & 0 & 4.102722 & -2.116877 & -0.681621 \\
\hline 70 & 1 & 0 & 2.850367 & -3.331840 & -0.422957 \\
\hline 71 & 1 & 0 & 3.175646 & -1.487446 & -3.026332 \\
\hline 72 & 1 & 0 & 1.421235 & -1.266015 & -3.226981 \\
\hline 73 & 1 & 0 & 2.071027 & -2.842483 & -2.777618 \\
\hline
\end{tabular}

4

\begin{tabular}{|c|c|c|c|c|c|}
\hline \multirow{2}{*}{$\begin{array}{l}\text { Center } \\
\text { Number }\end{array}$} & \multirow{2}{*}{$\begin{array}{l}\text { Atomic } \\
\text { Number }\end{array}$} & \multirow{2}{*}{$\begin{array}{l}\text { Atomic } \\
\text { Type }\end{array}$} & \multicolumn{3}{|c|}{ Coordinates (Angstroms) } \\
\hline & & & $\mathrm{X}$ & $\mathrm{Y}$ & Z \\
\hline & 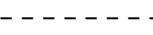 & & ---------- & --------- & --------- \\
\hline 1 & 6 & 0 & -3.085872 & -2.027072 & -1.631285 \\
\hline 2 & 14 & 0 & -2.683389 & -0.969186 & -0.099053 \\
\hline 3 & 6 & 0 & -3.435205 & -1.878057 & 1.398099 \\
\hline 4 & 6 & 0 & -0.858507 & -0.780503 & 0.114443 \\
\hline 5 & 6 & 0 & -0.043794 & -2.040443 & 0.462955 \\
\hline 6 & 6 & 0 & 1.451649 & -1.954881 & 0.256593 \\
\hline 7 & 6 & 0 & 2.146182 & -0.878087 & -0.152878 \\
\hline 8 & 7 & 0 & 3.540996 & -0.761615 & -0.239378 \\
\hline 9 & 6 & 0 & 4.314801 & -1.906866 & 0.203979 \\
\hline 10 & 15 & 0 & 0.004339 & 0.636660 & 0.073226 \\
\hline 11 & 8 & 0 & 1.544790 & 0.324126 & -0.511165 \\
\hline 12 & 7 & 0 & 0.345780 & 1.469231 & 1.510686 \\
\hline 13 & 6 & 0 & -0.022968 & 0.891439 & 2.799180 \\
\hline 14 & 7 & 0 & -0.507894 & 1.841324 & -0.961018 \\
\hline 15 & 6 & 0 & -0.502362 & 1.595843 & -2.400413 \\
\hline 16 & 6 & 0 & -3.613830 & 0.677847 & -0.282938 \\
\hline 17 & 6 & 0 & 1.469761 & 2.403441 & 1.579584 \\
\hline 18 & 6 & 0 & 4.067886 & -0.139360 & -1.453695 \\
\hline 19 & 6 & 0 & -0.967418 & 3.167418 & -0.572664 \\
\hline 20 & 1 & 0 & -4.161900 & -2.230668 & -1.708128 \\
\hline
\end{tabular}




\begin{tabular}{|c|c|c|c|c|c|}
\hline 21 & 1 & 0 & -2.571885 & -2.995774 & -1.599732 \\
\hline 22 & 1 & 0 & -2.773098 & -1.523086 & -2.553910 \\
\hline 23 & 1 & 0 & -4.500562 & -2.091180 & 1.241457 \\
\hline 24 & 1 & 0 & -3.347747 & -1.280714 & 2.314502 \\
\hline 25 & 1 & 0 & -2.934585 & -2.836509 & 1.583653 \\
\hline 26 & 1 & 0 & -0.226724 & -2.362713 & 1.504734 \\
\hline 27 & 1 & 0 & -0.410672 & -2.881668 & -0.149006 \\
\hline 28 & 1 & 0 & 2.006303 & -2.862145 & 0.469272 \\
\hline 29 & 1 & 0 & 5.373542 & -1.629573 & 0.217370 \\
\hline 30 & 1 & 0 & 4.199182 & -2.791733 & -0.447384 \\
\hline 31 & 1 & 0 & 4.016416 & -2.185236 & 1.218732 \\
\hline 32 & 1 & 0 & -0.385031 & 1.678586 & 3.474394 \\
\hline 33 & 1 & 0 & 0.832688 & 0.393556 & 3.280576 \\
\hline 34 & 1 & 0 & -0.816796 & 0.156259 & 2.654409 \\
\hline 35 & 1 & 0 & -1.509301 & 1.731922 & -2.818799 \\
\hline 36 & 1 & 0 & -0.178961 & 0.575105 & -2.608423 \\
\hline 37 & 1 & 0 & 0.179192 & 2.292771 & -2.909073 \\
\hline 38 & 1 & 0 & -4.692534 & 0.484725 & -0.341262 \\
\hline 39 & 1 & 0 & -3.327848 & 1.215514 & -1.193521 \\
\hline 40 & 1 & 0 & -3.446786 & 1.347026 & 0.569443 \\
\hline 41 & 1 & 0 & 1.234168 & 3.218710 & 2.276566 \\
\hline 42 & 1 & 0 & 1.665847 & 2.836712 & 0.596977 \\
\hline 43 & 1 & 0 & 2.388609 & 1.905663 & 1.921999 \\
\hline 44 & 1 & 0 & 5.109166 & 0.151832 & -1.280238 \\
\hline 45 & 1 & 0 & 3.490440 & 0.754326 & -1.688898 \\
\hline 46 & 1 & 0 & 4.037545 & -0.818322 & -2.323966 \\
\hline 47 & 1 & 0 & -1.977294 & 3.346820 & -0.965861 \\
\hline 48 & 1 & 0 & -0.302689 & 3.949426 & -0.969606 \\
\hline 49 & 1 & 0 & -0.998750 & 3.249178 & 0.513603 \\
\hline \multicolumn{6}{|c|}{ - - - - - - - - - - - - - - - - - - - - - - - - - - - - - - - - - - - - - - - - - - - - - - - - - - - - - - - - - - - - - } \\
\hline \multicolumn{6}{|l|}{5} \\
\hline \multicolumn{6}{|c|}{ 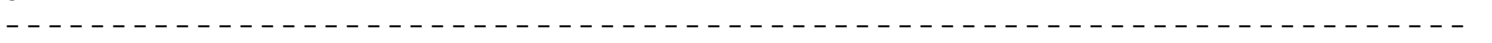 } \\
\hline Center & Atomic & Atomic & \multicolumn{3}{|c|}{ Coordinates (Angstroms) } \\
\hline Number & Number & Type & $\mathrm{X}$ & \multicolumn{2}{|c|}{$\mathrm{Y} \quad \mathrm{Z}$} \\
\hline------1 & ------ & & --------1 & -------- & -------- \\
\hline 1 & 6 & 0 & 0.997968 & -0.668726 & -0.256015 \\
\hline 2 & 14 & 0 & 2.695385 & -1.289700 & 0.069909 \\
\hline 3 & 6 & 0 & 2.693754 & -2.628252 & 1.427461 \\
\hline 4 & 15 & 0 & 0.135699 & 0.774993 & -0.146746 \\
\hline 5 & 7 & 0 & 0.259942 & 2.066778 & -1.261312 \\
\hline 6 & 6 & 0 & 1.554538 & 2.637014 & -1.592350 \\
\hline 7 & 7 & 0 & 0.196133 & 1.558073 & 1.357007 \\
\hline 8 & 6 & 0 & -0.267368 & 2.929236 & 1.560142 \\
\hline 9 & 6 & 0 & 0.078447 & 0.751071 & 2.575136 \\
\hline 10 & 6 & 0 & 3.456714 & -2.096333 & -1.478799 \\
\hline 11 & 6 & 0 & 3.844516 & 0.116127 & 0.635571 \\
\hline 12 & 6 & 0 & -0.895306 & 2.830126 & -1.708578 \\
\hline 13 & 1 & 0 & 3.692408 & -3.061139 & 1.570366 \\
\hline 14 & 1 & 0 & 2.367409 & -2.219568 & 2.391944 \\
\hline 15 & 1 & 0 & 2.013502 & -3.451894 & 1.175490 \\
\hline 16 & 1 & 0 & 1.598051 & 2.864426 & -2.666717 \\
\hline 17 & 1 & 0 & 1.767906 & 3.567752 & -1.040572 \\
\hline 18 & 1 & 0 & 2.344709 & 1.914507 & -1.370344 \\
\hline 19 & 1 & 0 & -1.350778 & 2.969283 & 1.743331 \\
\hline 20 & 1 & 0 & 0.247546 & 3.352478 & 2.431582 \\
\hline 21 & 1 & 0 & -0.025843 & 3.547783 & 0.696001 \\
\hline 22 & 1 & 0 & -0.962913 & 0.710643 & 2.922340 \\
\hline 23 & 1 & 0 & 0.427791 & -0.262905 & 2.375959 \\
\hline 24 & 1 & 0 & 0.702527 & 1.190904 & 3.363628 \\
\hline 25 & 1 & 0 & 4.452048 & -2.510941 & -1.272558 \\
\hline 26 & 1 & 0 & 2.831502 & -2.918407 & -1.850290 \\
\hline 27 & 1 & 0 & 3.557663 & -1.369570 & -2.294039 \\
\hline 28 & 1 & 0 & 4.798285 & -0.289168 & 0.996290 \\
\hline
\end{tabular}




$\begin{array}{rrrrrr}29 & 1 & 0 & 4.076194 & 0.810089 & -0.181552 \\ 30 & 1 & 0 & 3.402361 & 0.700184 & 1.451167 \\ 31 & 1 & 0 & -0.915722 & 2.886698 & -2.807248 \\ 32 & 1 & 0 & -1.820980 & 2.358333 & -1.367978 \\ 33 & 1 & 0 & -0.892384 & 3.862150 & -1.322046 \\ 34 & 6 & 0 & -0.228043 & -1.454621 & -0.777378 \\ 35 & 6 & 0 & -1.266374 & -0.268836 & -0.783697 \\ 36 & 6 & 0 & -2.524607 & -0.331024 & 0.069226 \\ 37 & 8 & 0 & -2.745589 & 0.538696 & 0.917211 \\ 38 & 7 & 0 & -3.408237 & -1.356732 & -0.152220 \\ 39 & 6 & 0 & -4.606588 & -1.452761 & 0.669341 \\ 40 & 6 & 0 & -3.289144 & -2.356755 & -1.199881 \\ 41 & 1 & 0 & -0.119525 & -1.886305 & -1.785795 \\ 42 & 1 & 0 & -0.548727 & -2.271727 & -0.112448 \\ 43 & 1 & 0 & -1.538416 & 0.018298 & -1.806653 \\ 44 & 1 & 0 & -4.658861 & -2.438247 & 1.150090 \\ 45 & 1 & 0 & -4.568703 & -0.673500 & 1.428815 \\ 46 & 1 & 0 & -5.508239 & -1.320495 & 0.055937 \\ 47 & 1 & 0 & -2.432374 & -2.155225 & -1.840381 \\ 48 & 1 & 0 & -3.174299 & -3.362144 & -0.772479 \\ 49 & 1 & 0 & -4.194212 & -2.355393 & -1.822472 \\ -----------------1\end{array}$

